\title{
The arbitrary-order virtual element method for linear elastodynamics models: convergence, stability and dispersion-dissipation analysis
}

\author{
Paola F. Antonietti ${ }^{1}$ | Gianmarco Manzini² $^{2}$ | Ilario Mazzieri ${ }^{1}$ | \\ Hashem M. Mourad ${ }^{3}$ | Marco Verani ${ }^{1}$
}

${ }^{1}$ MOX, Dipartimento di Matematica, Politecnico di Milano, Milano, Italy

${ }^{2}$ Group T-5, Theoretical Division, Los Alamos National Laboratory, Los Alamos, New Mexico, USA

${ }^{3}$ Group T-3, Theoretical Division, Los Alamos National Laboratory, Los Alamos, New Mexico, USA

\section{Correspondence}

Gianmarco Manzini, Los Alamos National Laboratory, Los Alamos, NM.

Email: gmanzini@lanl.gov

\section{Funding information}

Laboratory Directed Research and Development, Grant/Award Number: 20180428ER, 20170033DR; PRIN - Virtual Element Methods: Analysis and Applications, Grant/Award Number: 201744KLJL

\begin{abstract}
We design the conforming virtual element method for the numerical approximation of the two-dimensional elastodynamics problem. We prove stability and convergence of the semidiscrete approximation and derive optimal error estimates under $h$ - and $p$-refinement in both the energy and the $L^{2}$ norms. The performance of the proposed virtual element method is assessed on a set of different computational meshes, including nonconvex cells up to order four in the $h$-refinement setting. Exponential convergence is also experimentally observed under $p$-refinement. Finally, we present a dispersion-dissipation analysis for both the semidiscrete and fully discrete schemes, showing that polygonal meshes behave as classical simplicial/quadrilateral grids in terms of dispersion-dissipation properties.
\end{abstract}

\section{K E Y W O R D S}

elastodynamics, high-order methods, polygonal meshes, virtual element method

\section{I INTRODUCTION}

In recent years, numerical modeling of elastic waves propagation problems through the elastodynamics equation has undergone a constantly increasing interest in the mathematical and geophysics engineering community. One of the most employed numerical technique is the spectral element method that has successfully been applied to the elastodynamics equation. ${ }^{1,2}$ Elastic waves propagation problems have been treated numerically by applying the discontinuous Galerkin (DG) and the discontinuous Galerkin spectral element method ${ }^{3-5}$ to the displacement formulation and the stress-velocity formulation. ${ }^{6}$ High-order DG methods for elastic and elastoacoustic wave propagation problems have been extended to arbitrarily shaped polygonal/polyhedral grids ${ }^{7,8}$ to further enhance the geometrical flexibility of the DG approach while guaranteeing low dissipation and dispersion errors. Recently, the lowest-order virtual element method (VEM) has been applied for the solution of the elastodynamics equation on nonconvex polygonal meshes. ${ }^{9,10}$

Studying the elastodynamic behavior of structures with complicated geometrical features is often of interest in many practical situations, for example, in aerospace and power-generation applications. Traditionally, triangular-and in 3D, tetrahedral-elements have been the only available option when it came to the spatial discretization of such structures. This is problematic because low-order triangles/tetrahedra are known to overestimate the structure's stiffness (and hence its natural frequencies and the wave propagation speed within it), especially with nearly 
incompressible materials. In addition, very small triangular elements are often needed to resolve intricate features of the geometry, which then requires a very small time step to be used in view of the CFL stability condition. Polygonal elements can alleviate such difficulties, since they often obviate the need to refine the spatial discretization even in the presence of complicated/intricate geometrical features. This is especially true if the underlying numerical method allows nonconvex polygonal elements to be used efficiently, as is the case with the virtual element method.

The VEM, that can be seen as a variational reformulation of the nodal mimetic finite difference (MFD) method, ${ }^{11}$ was originally proposed as a conforming method for solving elliptic boundary-value problems on polytopal meshes ${ }^{12}$ and later extended to nonconforming formulations, ${ }^{13-17}$ and to higher-order differential equations, ${ }^{16,18}$ and to "p/hp" formulations, also using adaptive mesh refinements. ${ }^{19}$ The VEM provides a very effective and flexible setting for designing arbitrary-order accurate numerical methods suited to polygonal and polyhedral meshes with respect to straightforward generalization of the finite element method as in the polygonal FEM. ${ }^{20}$ In the last few years, great amount of work has also been devoted to the numerical modeling of linear and nonlinear elasticity problems and materials. For example, it is worth mentioning the VEM for plate bending problems ${ }^{21,22}$ and VEM for the stress-displacement formulation of plane elasticity problems, ${ }^{23}$ plane elasticity problems based on the Hellinger-Reissner principle, ${ }^{24}$ mixed virtual element method for a pseudostress-based formulation of linear elasticity, ${ }^{25}$ nonconforming virtual element method for elasticity problems, ${ }^{26}$ linear ${ }^{27}$ and nonlinear elasticity, ${ }^{28}$ contact problems ${ }^{29}$ and frictional contact problems including large deformations, ${ }^{30}$ elastic and inelastic problems on polytope meshes, ${ }^{31}$ compressible and incompressible finite deformations,${ }^{32}$ finite elastoplastic deformations, ${ }^{33-35}$ virtual element method for coupled thermoelasticity in Abaqus, ${ }^{36}$ a priori and a posteriori error estimates for a virtual element spectral analysis for the elasticity equations, ${ }^{37}$ virtual element method for transversely isotropic elasticity. ${ }^{38}$ In this article, we are aimed at investigating both theoretically and numerically the performance of the high-order virtual element method for the numerical modeling of wave propagation phenomena in elastic media. In particular, we prove the stability and the convergence of the semidiscrete approximation in the energy norm and derive error estimates. The performance of the method, in terms of convergence, stability, and dispersion-dissipation analysis, is assessed on a set of different computational meshes. Exponential convergence is also experimentally seen in the $p$-refinement setting. With the aim of focusing the reader on the novelties of the present work, we note that at the time of writing, only one work has been published on a somewhat similar, though simpler problem, namely the numerical approximation of the wave equation in the framework of the conforming virtual element method using the elliptic projection operator to define the stiffness matrix. ${ }^{39}$ However, our work is substantially different since we consider a more complex mathematical model, a diverse range of applications, a different discretization of the stiffness matrix, which is here based on orthogonal projections of the symmetric gradients and not on the elliptic projector, and, consequently, a substantially different strategy for the analysis both in $L^{2}$ and energy norm. Moreover, the dispersion-dissipation analysis presented in the last part of the our work is specifically carried out for the elastodynamics problem here considered.

The outline of the article is as follows. We conclude this introductory section with a subsection introducing the notation used in this article. Then, in Section 2, we introduce the model problem and its virtual element approximation. In Section 3, we present the design of the VEM. In Section 4, we discuss the convergence of the VEM and derive suitable bounds for the approximation error. In Section 5, we investigate the performance of the method on a set of suitable numerical experiments. In particular, in Section 5.1 we show the optimal convergence properties of the VEM by using a manufactured solution on three mesh families with different difficulties, and in Section 5.2, we carry out an experimental investigation à la Von Neumann on the stability and dispersion-dissipation properties of the method. In Section 6, we offer our final remarks and conclusions.

\subsection{Notation of functional spaces and technicalities}

We use the standard definitions and notation of Sobolev spaces, norms, and seminorms. ${ }^{40}$ Let $k$ be a nonnegative integer number. The Sobolev space $H^{k}(\omega)$ consists of all square integrable functions with all square integrable weak derivatives up to order $k$ that are defined on the open bounded connected subset $\omega$ of $\mathbb{R}^{2}$. As usual, if $k=0$, we prefer the notation $L^{2}(\omega)$. Norm and seminorm in $H^{k}(\omega)$ are denoted by $\|\cdot\|_{k, \omega}$ and $|\cdot|_{k, \omega}$, respectively, and $(\cdot, \cdot)_{\omega}$ denotes the $L^{2}$-inner product. We omit the subscript $\omega$ when $\omega$ is the whole computational domain $\Omega$. 
Given the mesh partitioning $\Omega_{h}=\{\mathrm{P}\}$ of the domain $\Omega$ into elements $\mathrm{P}$, we define the broken (scalar) Sobolev space for any integer $k>0$

$$
H^{k}\left(\Omega_{h}\right)=\prod_{\mathrm{P} \in \Omega_{h}} H^{k}(\mathrm{P})=\left\{v \in L^{2}(\Omega): v_{\mid \mathrm{P}} \in H^{k}(\mathrm{P})\right\}
$$

which we endow with the broken $H^{k}$-norm

$$
\|v\|_{k, h}^{2}=\sum_{\mathrm{P} \in \Omega_{h}}\|v\|_{k, \mathrm{P}}^{2} \quad \forall v \in H^{k}\left(\Omega_{h}\right)
$$

and, for $k=1$, with the broken $H^{1}$-seminorm

$$
|v|_{1, h}^{2}=\sum_{\mathrm{P} \in \Omega_{h}}\|\nabla v\|_{0, \mathrm{P}}^{2} \quad \forall v \in H^{1}\left(\Omega_{h}\right) .
$$

We denote the linear space of polynomials of degree up to $\ell$ defined on $\omega$ by $\mathbb{P}_{\ell}(\omega)$, with the useful conventional notation that $\mathbb{P}_{-1}(\omega)=\{0\}$. We denote the space of two-dimensional vector polynomials of degree up to $\ell$ on $\omega$ by $\left[\mathbb{P}_{\ell}(\omega)\right]^{2}$; the space of symmetric $2 \times 2$-sized tensor polynomials of degree up to $\ell$ on $\omega$ by $\mathbb{P}_{\ell \text {,sym }}^{2 \times 2}(\omega)$. Space $\mathbb{P}_{\ell}(\omega)$ is the span of the finite set of scaled monomials of degree up to $\ell$, that are given by

$$
\mathcal{M}_{\ell}(\omega)=\left\{\left(\frac{\mathbf{x}-\mathbf{x}_{\omega}}{h_{\omega}}\right)^{\alpha} \text { with }|\alpha| \leq \ell\right\}
$$

where

- $\mathbf{x}_{\omega}$ denotes the center of gravity of $\omega$ and $h_{\omega}$ its characteristic length, as, for instance, the edge length or the cell diameter for $d=1,2$;

- $\alpha=\left(\alpha_{1}, \alpha_{2}\right)$ is the two-dimensional multiindex of nonnegative integers $\alpha_{i}$ with degree $|\alpha|=\alpha_{1}+\alpha_{2} \leq \ell$ and such that $\mathbf{x}^{\alpha}=x_{1}^{\alpha_{1}} x_{2}^{\alpha_{2}}$ for any $\mathbf{x} \in \mathbb{R}^{2}$.

We will also use the set of scaled monomials of degree exactly equal to $\ell$, denoted by $\mathcal{M}_{\ell}^{*}(\omega)$ and obtained by setting $|\alpha|=\ell$ in the definition above.

Finally, we use the letter $C$ in the error estimates to denote a strictly positive constant whose value can change at any instance and that is independent of the discretization parameters such as the mesh size $h$. Note that $C$ may depend on the constants of the model equations or the variational problem, like the coercivity and continuity constants, or even constants that are uniformly defined for the family of meshes of the approximation while $h \rightarrow 0$, such as the mesh regularity constant, the stability constants of the discrete bilinear forms, and so on. Whenever it is convenient, we will simplify the notation by using expressions like $x \lesssim y$ and $x \gtrsim y$ to mean that $x \leq C y$ and $x \geq C y$, respectively, $C$ being the generic constant in the sense defined above.

\section{2 | MODEL PROBLEM AND VIRTUAL ELEMENT FORMULATION}

We consider an elastic body occupying the open, bounded polygonal domain denoted by $\Omega \in \mathbb{R}^{2}$ with boundary denoted by $\Gamma=\partial \Omega$. We assume that boundary $\Gamma$ can be split into the two disjoint subsets $\Gamma_{D}$ and $\Gamma_{N}$, so that $\bar{\Gamma}=\bar{\Gamma}_{D} \cup \bar{\Gamma}_{N}$ and $\Gamma_{D} \cap \Gamma_{N}=\emptyset$. For the well-posedness of the mathematical model, we further require that the one-dimensional Lebesgue measure (length) of $\Gamma_{D}$ is nonzero, that is, $\left|\Gamma_{D}\right|>0$. Let $T>0$ denote the final time. We

consider the external load $\mathbf{f} \in L^{2}\left((0, T) ;\left[L^{2}(\Omega)\right]^{2}\right)$, the boundary function $\mathbf{g}_{N} \in C^{1}\left((0, T) ;\left[H_{0, \Gamma_{N}}^{\frac{1}{2}}\right]^{2}\right)$, and the initial functions $\mathbf{u}_{0} \in\left[H_{0, \Gamma_{D}}^{1}(\Omega)\right]^{2}, \mathbf{u}_{1} \in\left[L^{2}(\Omega)\right]^{2}$. For such time-dependent vector fields, we may indicate the dependence on time explicitly, for example, $\mathbf{f}(t):=\mathbf{f}(\cdot, t) \in\left[L^{2}(\Omega)\right]^{2}$, or drop it out to ease the notation when it is obvious from the context. 
The equations governing the two-dimensional initial/boundary-value problem of linear elastodynamics for the displacement vector $\mathbf{u}: \Omega \times[0, T] \rightarrow \mathbb{R}^{2}$ are:

$$
\begin{aligned}
\rho \ddot{\mathbf{u}}-\nabla \cdot \boldsymbol{\sigma}(\mathbf{u})=\mathbf{f} & \text { in } \Omega \times(0, T], \\
\mathbf{u}=\mathbf{0} & \text { on } \Gamma_{D} \times(0, T], \\
\boldsymbol{\sigma}(\mathbf{u}) \mathbf{n}=\mathbf{g}_{N} & \text { on } \Gamma_{N} \times(0, T], \\
\mathbf{u}=\mathbf{u}_{0} & \text { in } \Omega \times\{0\}, \\
\dot{\mathbf{u}}=\mathbf{u}_{1} & \text { in } \Omega \times\{0\} .
\end{aligned}
$$

Here, $\rho$ is the mass density, which we suppose to be a strictly positive and uniformly bounded function and $\sigma(\mathbf{u})$ is the stress tensor. In (4) we assume homogeneous Dirichlet boundary conditions on $\Gamma_{D}$. This assumption is made only to ease the exposition and the analysis, as our numerical method is easily extendable to treat the case of nonhomogeneous Dirichlet boundary conditions.

We denote the space of symmetric $2 \times 2$-sized real-valued tensors by $\mathbb{R}_{\text {sym }}^{2 \times 2}$ and assume that the stress tensor $\sigma: \Omega \times$ $[0, T] \rightarrow \mathbb{R}_{\text {sym }}^{2 \times 2}$ is expressed, according to Hooke's law, by $\sigma(\mathbf{u})=\mathcal{D} \varepsilon(\mathbf{u})$, where $\varepsilon(\mathbf{u})$ denotes the symmetric gradient of $\mathbf{u}$, that is, $\varepsilon(\mathbf{u})=\left(\nabla \mathbf{u}+(\nabla \mathbf{u})^{T}\right) / 2$, and $\mathcal{D}=\mathcal{D}(\mathbf{x}): \mathbb{R}_{\text {sym }}^{2 \times 2} \rightarrow \mathbb{R}_{\text {sym }}^{2 \times 2}$ is the stiffness tensor

$$
D \tau=2 \mu \tau+\lambda \operatorname{tr}(\tau) \mid
$$

for all $\tau \in \mathbb{R}_{\mathrm{sym}}^{2 \times 2}$. In this definition, I and $\operatorname{tr}(\cdot)$ are the identity matrix and the trace operator; $\lambda$ and $\mu$ are the first and second Lamé coefficients, which we assume to be in $L^{\infty}(\Omega)$ and nonnegative. The compressional (P) and shear (S) wave velocities of the medium are respectively obtained through the relations $c_{P}=\sqrt{(\lambda+2 \mu) / \rho}$ and $c_{S}=\sqrt{\mu / \rho}$.

Let $\mathbf{V}=\left[H_{\Gamma_{D}}^{1}(\Omega)\right]^{2}$ be the space of $H^{1}$ vector valued functions with null trace on $\Gamma_{D}$. We consider the two bilinear forms $m(\cdot, \cdot), a(\cdot, \cdot): \mathbf{V} \times \mathbf{V} \rightarrow \mathbb{R}$ defined as

$$
\begin{gathered}
m(\mathbf{w}, \mathbf{v})=\int_{\Omega} \rho \mathbf{w} \cdot \mathbf{v} d V \quad \forall \mathbf{w}, \mathbf{v} \in \mathbf{V} \\
a(\mathbf{w}, \mathbf{v})=\int_{\Omega} \boldsymbol{\sigma}(\mathbf{w}): \varepsilon(\mathbf{v}) d V \quad \forall \mathbf{w}, \mathbf{v} \in \mathbf{V},
\end{gathered}
$$

and the linear functional $F(\cdot): \mathbf{V} \rightarrow \mathbb{R}$ defined as

$$
F(\mathbf{v})=\int_{\Omega} \mathbf{f} \cdot \mathbf{v} d V+\int_{\Gamma_{N}} \mathbf{g}_{N} \cdot \mathbf{v} \quad \forall \mathbf{v} \in \mathbf{V}
$$

The variational formulation of the linear elastodynamics equations reads as: For all $\mathrm{t} \in(0, \mathrm{~T}]$ find $\mathbf{u}(\mathrm{t}) \in \mathbf{V}$ such that for $\mathrm{t}=0$ it holds that $\mathbf{u}(0)=\mathbf{u}_{0}$ and $\mathbf{\mathbf { u }}(0)=\mathbf{u}_{1}$ and

$$
m(\ddot{\mathbf{u}}, \mathbf{v})+a(\mathbf{u}, \mathbf{v})=F(\mathbf{v}) \quad \forall \mathbf{v} \in \mathbf{V}
$$

As shown, for example, by Raviart and Thomas (see theorem 8-3.141) the variational problem (12) is well posed and its unique solution satisfies $\mathbf{u} \in C^{0}((0, T] ; \mathbf{V}) \cap C^{1}\left((0, T] ;\left[L^{2}(\Omega)\right]^{2}\right)$.

The virtual element approximation of problem (12) relies on the virtual element space $\mathbf{V}_{k}^{h}$, which is a subspace of $\left[H_{\Gamma_{D}}^{1}(\Omega)\right]^{2}$. Space $\mathbf{V}_{k}^{h}$ is built upon the scalar conforming space. ${ }^{12}$ For the sake of completeness, we briefly review the construction of the scalar space in the next section. Then, we consider the virtual element bilinear forms $m_{h}(\cdot, \cdot)$ and $a_{h}(\cdot, \cdot)$, which approximate the bilinear forms $m(\cdot, \cdot)$ and $a(\cdot, \cdot)$, and the virtual element functional $F_{h}(\cdot)$, which approximates the linear functional $F(\cdot)$. The definition of $m_{h}(\cdot, \cdot), a_{h}(\cdot, \cdot)$, and $F_{h}(\cdot)$ and the discussion of their properties is addressed in the next section. 
The semidiscrete virtual element approximation of (12) read as: For all $\mathrm{t} \in(0, \mathrm{~T}]$ find $\mathbf{u}_{h}(t) \in \mathbf{V}_{k}^{h}$ such that for $\mathrm{t}=0$ it holds that $\mathbf{u}_{\mathrm{h}}(0)=\left(\mathbf{u}_{0}\right)_{I}$ and $\dot{\mathbf{u}}_{h}(0)=\left(\mathbf{u}_{1}\right)_{I}$ and

$$
m_{h}\left(\ddot{\mathbf{u}}_{h}, \mathbf{v}_{h}\right)+a_{h}\left(\mathbf{u}_{h}, \mathbf{v}_{h}\right)=F_{h}\left(\mathbf{v}_{h}\right) \quad \forall \mathbf{v}_{h} \in \mathbf{V}_{k}^{h} .
$$

Here, $\mathbf{u}_{h}(t)$ is the virtual element approximation of $\mathbf{u}$ and $\mathbf{v}_{h}$ the generic test function in $\mathbf{V}_{k}^{h}$, while $\left(\mathbf{u}_{0}\right)_{I}$ and $\left(\mathbf{u}_{1}\right)_{I}$ are the virtual element interpolants of the initial solution functions $\mathbf{u}(0)$ and $\dot{\mathbf{u}}(0)$, respectively.

Time integration is performed by applying the leap-frog time marching scheme ${ }^{42}$ to the second derivative in time $\ddot{\mathbf{u}}_{h}$. To this end, we subdivide the interval $(0, T]$ into $N_{T}$ subintervals of amplitude $\Delta t=T / N_{T}$ and at every time level $t^{n}=n \Delta t$ we consider the variational problem for $n \geq 1$ :

$$
m_{h}\left(\mathbf{u}_{h}^{n+1}, \mathbf{v}_{h}\right)-2 m_{h}\left(\mathbf{u}_{h}^{n}, \mathbf{v}_{h}\right)+m_{h}\left(\mathbf{u}_{h}^{n-1}, \mathbf{v}_{h}\right)+\Delta t^{2} a_{h}\left(\mathbf{u}_{h}^{n}, \mathbf{v}_{h}\right)=\Delta t^{2} F_{h}^{n}\left(\mathbf{v}_{h}\right) \quad \forall \mathbf{v}_{h} \in V_{k}^{h}
$$

and initial step

$$
m_{h}\left(\mathbf{u}_{h}^{1}, \mathbf{v}_{h}\right)-m_{h}\left(\mathbf{u}_{0}, \mathbf{v}_{h}\right)-\Delta t m_{h}\left(\mathbf{u}_{1}, \mathbf{v}_{h}\right)+\frac{\Delta t^{2}}{2} a_{h}\left(\mathbf{u}_{0}, \mathbf{v}_{h}\right)=\frac{\Delta t^{2}}{2} F_{h}^{0}\left(\mathbf{v}_{h}\right) \quad \forall \mathbf{v}_{h} \in V_{k}^{h}
$$

The leap-frog scheme is second-order accurate, explicit, and conditionally stable. ${ }^{42}$ It is straightforward to show that these properties are inherited by the fully discrete scheme (14).

\section{3 | VIRTUAL ELEMENT APPROXIMATION}

The VEM proposed in this article is a vector extension of the VEM previously developed for scalar elliptic and parabolic problems, ${ }^{12-14,43}$ that we shortly review in this section. First, we introduce the family of mesh decompositions of the computational domain and the mesh regularity assumptions needed to prove the stability and convergence of the method. Then, we formulate the conforming virtual element spaces of various degree $k$ and present the degrees of freedom that are unisolvent in such spaces. Finally, we present the definition of the virtual element bilinear forms and discuss their properties.

\section{1 | Mesh definition and regularity assumptions}

Let $\mathcal{T}=\left\{\Omega_{h}\right\}_{h}$ be a family of decompositions of $\Omega$ into nonoverlapping polygonal elements $\mathrm{P}$ with nonintersecting boundary $\partial \mathrm{P}$, center of gravity $\mathbf{x}_{\mathrm{P}}$, two-dimensional measure (area) $|\mathrm{P}|$, and diameter $h_{\mathrm{P}}=\sup _{\mathbf{x}, \mathbf{y} \in \mathrm{P}}|\mathbf{x}-\mathbf{y}|$. The subindex $h$ that labels each mesh $\Omega_{h}$ is the maximum of the diameters $h_{\mathrm{P}}$ of the elements of that mesh. The boundary of P is formed by straight edges $e$ and the midpoint and length of each edge are denoted by $\mathbf{x}_{e}$ and $h_{e}$, respectively.

We denote the unit normal vector to the elemental boundary $\partial \mathrm{P}$ by $\mathbf{n}_{\mathrm{P}}$, and the unit normal vector to edge $e$ by $\mathbf{n}_{e}$. Vector $\mathbf{n}_{\mathrm{P}}$ points out of $\mathrm{P}$ and the orientation of $\mathbf{n}_{e}$ is fixed once and for all in every mesh

Now, we state the mesh regularity assumptions that are required for the convergence analysis.

(A0) Mesh regularity assumptions.

- There exists a positive constant $\rho$ independent of $h$ (and, hence, of $\Omega_{h}$ ) such that for every polygonal element P $\in \Omega_{h}$ it holds that

(i) $\mathrm{P}$ is star-shaped with respect to a disk with radius $\geq \rho h_{\mathrm{P}}$;

(ii) for every edge $e \in \partial \mathrm{P}$ it holds that $h_{e} \geq \rho h_{\mathrm{P}}$.

Remark 1. Star-shapedness property (i) implies that the elements are simply connected subsets of $\mathbb{R}^{2}$. Scaling property (ii) implies that the number of edges in each elemental boundary is uniformly bounded over the whole mesh family $\mathcal{T}$.

To conclude this section, we note that the above mesh assumptions are very general and, as observed from the first publications on the VEM, ${ }^{12}$ allow us to formulate the VEM on grids of polygonal elements having very general geometric shapes, for example, nonconvex elements or elements with hanging nodes. However, virtual element formulations under weaker mesh assumptions have been the object of recent investigations. ${ }^{44-46}$ 


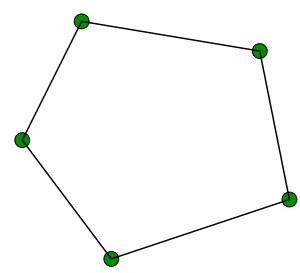

$(\mathrm{k}=1)$

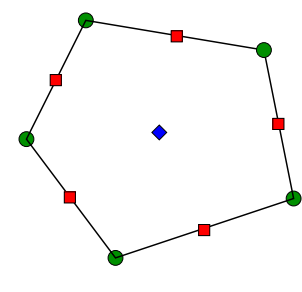

$(\mathrm{k}=2)$

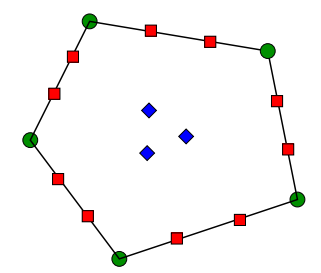

$(\mathrm{k}=3)$
F I G URE 1 The degrees of freedom of the scalar conforming virtual element spaces $V k h(\mathrm{P})$ defined on the pentagonal cell $\mathrm{P}$ for $k=1,2,3$

\section{2 | Virtual element space, degrees of freedom, and projection operators}

The global virtual element space is defined as

$$
\mathbf{V}_{k}^{h}:=\left\{\mathbf{v} \in \mathbf{V}: \mathbf{v}_{\mathrm{l}_{\mathrm{P}}} \in \mathbf{V}_{k}^{h}(\mathrm{P}) \text { for every } \mathrm{P} \in \Omega_{h}\right\}
$$

The construction of the local virtual element space is carried out along these three steps: $(i)$ we select the set of degrees of freedom that uniquely characterizes the functions of the local space; (ii) we introduce the elliptic projector onto the subspace of polynomials; (iii) we define the virtual element functions as the solution of a differential problem, also using the elliptic projector.

Let us move to the first step.

- Each virtual element function $v_{h}$ is uniquely characterized by

(C1) the values of $v_{h}$ at the vertices of P;

(C2) the moments of $v_{h}$ of order up to $k-2$ on each one-dimensional edge $e \in \partial \mathrm{P}$ :

$$
\frac{1}{|e|} \int_{e} v_{h} m d s, \quad \forall m \in \mathcal{M}_{k-2}(e), \forall e \in \partial \mathrm{P} ;
$$

(C3) the moments of $v_{h}$ of order up to $k-2$ on P:

$$
\frac{1}{|\mathrm{P}|} \int_{\mathrm{P}} v_{h} m d s, \quad \forall m \in \mathcal{M}_{k-2}(\mathrm{P}) .
$$

Figure 1 shows the degrees of freedom of the three scalar conforming virtual element spaces defined on a pentagonal cell for $k=1,2$, 3. Since we assume that $\mathbf{V}_{k}^{h}(\mathrm{P})=\left[V_{k}^{h}(\mathrm{P})\right]^{2}, V k h(\mathrm{P})$ being the local scalar conforming virtual element space, the degrees of freedom of each component of the vector-valued functions $\mathbf{v}_{h}$ are those described above.

In the second step, we introduce the elliptic projection operator $\Pi_{k}^{\nabla}: H^{1}(\mathrm{P}) \cap C^{0}(\overline{\mathrm{P}}) \rightarrow \mathbb{P}_{k}(\mathrm{P})$, so that the elliptic projection of a function $v_{h}$ is the polynomial of degree $k$ that satisfies the variational problem given by

$$
\int_{\mathrm{P}} \nabla \Pi_{k}^{\nabla} v_{h} \cdot \nabla q d V=\int_{\mathrm{P}} \nabla v_{h} \cdot \nabla q d V \quad \forall q \in \mathbb{P}_{k}(\mathrm{P})
$$

with the additional condition that

$$
\begin{gathered}
\int_{\partial \mathrm{P}} \Pi_{1}^{\nabla} v_{h} d V=\int_{\partial \mathrm{P}} v_{h} d V \quad \text { for } \quad k=1, \\
\int_{\mathrm{P}} \Pi_{k}^{\nabla} v_{h} d V=\int_{\mathrm{P}} v_{h} d V \quad \text { for } \quad k \geq 2 .
\end{gathered}
$$

The crucial property is that $\Pi_{k}^{\nabla} v_{h}$ is computable using only the information on $v_{h}$ provided by its degrees of freedom, that is, the values of the linear functionals (C1)-(C3).

In the third and final step, we define the conforming virtual element space of order $k \geq 1$ by

$$
\begin{aligned}
V_{k}^{h}(\mathrm{P}):=\left\{v_{h} \in H^{1}(\mathrm{P}):\right. & : v_{\left.h\right|_{\partial \mathrm{P}}} \\
& \in C(\partial \mathrm{P}), v_{\left.h\right|_{e}} \in \mathbb{P}_{k}(e) \quad \forall e \in \partial \mathrm{P}, \\
\Delta v_{h} & \left.\in \mathbb{P}_{k}(\mathrm{P}),\left(v_{h}-\Pi_{k}^{\nabla} v_{h}, \mu_{h}\right)_{\mathrm{P}}=0 \quad \forall \mu_{h} \in \mathbb{P}_{k}(\mathrm{P}) \backslash \mathbb{P}_{k-2}(\mathrm{P})\right\} .
\end{aligned}
$$


Remark 2. A few remarkable facts characterize these scalar functional spaces:

(i) their definition resorts to the enhancement strategy ${ }^{43}$ for the conforming VEM;

(ii) the degrees of freedom (C1)-(C3) are unisolvent ${ }^{12}$ in $V_{k}^{h}(\mathrm{P})$;

(iii) the scalar polynomial space $\mathbb{P}_{k}(\mathrm{P})$ is a subset of $V_{k}^{h}(\mathrm{P})$;

(iv) the $L^{2}$-orthogonal projections $\Pi_{k}^{0} v_{h} \in \mathbb{P}_{k}(\mathrm{P})$ and $\Pi_{k-1}^{0} \nabla v_{h} \in\left[\mathbb{P}_{k-1}(\mathrm{P})\right]^{2}$ are computable for all $v_{h} \in V_{k}^{h}$ using only the degrees of freedom of $v_{h}{ }^{43}$

From (iv), we readily find that for all $\mathbf{v}_{h} \in \mathbf{V}_{k}^{h}$ the $L^{2}$-orthogonal projections $\Pi_{k}^{0} \mathbf{v}_{h} \in\left[\mathbb{P}_{k}(\mathrm{P})\right]^{2}$ and $\Pi_{k-1}^{0} \varepsilon\left(\mathbf{v}_{h}\right) \in$ $\mathbb{P}_{k-1, \mathrm{sym}}^{2 \times 2}(\mathrm{P})$ are also computable (without any approximation) using only the degrees of freedom of $\mathbf{v}_{h}$. In particular, the latter one is the solution of the finite dimensional variational problem:

$$
\int_{\mathrm{P}} \Pi_{k-1}^{0}\left(\varepsilon\left(\mathbf{v}_{h}\right)\right): \varepsilon^{p} d V=\int_{\mathrm{P}} \varepsilon\left(\mathbf{v}_{h}\right): \varepsilon^{p} d V \quad \forall \varepsilon^{p} \in \mathbb{P}_{k-1, \mathrm{sym}}^{2 \times 2}(\mathrm{P})
$$

that is, the $L^{2}$-projection of the symmetric gradient $\varepsilon\left(\mathbf{v}_{h}\right)$ onto $\mathbb{P}_{k-1, \text { sym }}^{2 \times 2}(\mathrm{P})$, that (we recall) is the space of symmetric $2 \times 2$-sized tensor-valued polynomials of degree up to $k-1$.

Finally, the degrees of freedom of the global space $\mathbf{V}_{k}^{h}$ are provided by collecting all the local degrees of freedom. We note that the vertex values are the same for all the elements to which a vertex belongs. Similarly, the edge values at internal edges (i.e., edges shared by two mesh elements) are the same for the two elements to which an edge belongs. The unisolvence of such degrees of freedom for the global space $\mathbf{V}_{k}^{h}$ is an immediate consequence of the unisolvence of the local degrees of freedom for the elemental spaces $V_{k}^{h}(\mathrm{P})$.

\section{3 | Virtual element bilinear forms}

In the virtual element setting, we define the bilinear forms $m_{h}(\cdot, \cdot)$ and $a_{h}(\cdot, \cdot)$ as the sum of elemental contributions, which are denoted by $m_{h}^{P}(\cdot, \cdot)$ and $a_{h}^{P}(\cdot, \cdot)$, respectively:

$$
\begin{gathered}
m_{h}(\cdot, \cdot): \mathbf{V}_{k}^{h} \times \mathbf{V}_{k}^{h} \rightarrow \mathbb{R}, \quad \text { with } \quad m_{h}\left(\mathbf{v}_{h}, \mathbf{w}_{h}\right)=\sum_{\mathrm{P} \in \Omega_{h}} m_{h}^{P}\left(\mathbf{v}_{h}, \mathbf{w}_{h}\right), \\
a_{h}(\cdot, \cdot): \mathbf{V}_{k}^{h} \times \mathbf{V}_{k}^{h} \rightarrow \mathbb{R}, \quad \text { with } \quad a_{h}\left(\mathbf{v}_{h}, \mathbf{w}_{h}\right)=\sum_{\mathrm{P} \in \Omega_{h}} a_{h}^{P}\left(\mathbf{v}_{h}, \mathbf{w}_{h}\right) .
\end{gathered}
$$

The local bilinear form $m_{h}^{P}(\cdot, \cdot)$ is given by

$$
m_{h}^{P}\left(\mathbf{v}_{h}, \mathbf{w}_{h}\right)=\int_{\mathrm{P}} \rho \Pi_{k}^{0} \mathbf{v}_{h} \cdot \Pi_{k}^{0} \mathbf{w}_{h} d V+S_{m}^{\mathrm{P}}\left(\mathbf{v}_{h}, \mathbf{w}_{h}\right),
$$

where $S_{m}^{P}(\cdot, \cdot)$ is the local stabilization term. The bilinear form $m_{h}^{P}$ depends on the orthogonal projections $\Pi_{k}^{0} \mathbf{v}_{h}$ and $\Pi_{k}^{0} \mathbf{w}_{h}$, which are computable from the degrees of freedom of $\mathbf{v}_{h}$ and $\mathbf{w}_{h}$, respectively, see the previous section. The local form $S_{m}^{P}(\cdot, \cdot): \mathbf{V}_{k}^{h} \times \mathbf{V}_{k}^{h} \rightarrow \mathbb{R}$ can be any symmetric and coercive bilinear form that is computable from the degrees of freedom and for which there exist two strictly positive real constants $\sigma_{*}$ and $\sigma^{*}$ such that

$$
\sigma_{*} m^{\mathrm{P}}\left(\mathbf{v}_{h}, \mathbf{v}_{h}\right) \leq S_{m}^{\mathrm{P}}\left(\mathbf{v}_{h}, \mathbf{v}_{h}\right) \leq \sigma^{*} m^{\mathrm{P}}\left(\mathbf{v}_{h}, \mathbf{v}_{h}\right) \quad \mathbf{v}_{h} \in \operatorname{ker}\left(\Pi_{k}^{0}\right) \cap \mathbf{V}_{k}^{h}(\mathrm{P}) .
$$

We can define computable stabilizations $S_{m}^{\mathrm{P}}(\cdot, \cdot)$ by resorting to the two-dimensional implementations of the effective choices for the scalar case proposed in the literature. ${ }^{47,48}$ The one used in our implementation of the method is discussed in Subsection 3.5.

The discrete bilinear form $m_{h}^{P}(\cdot, \cdot)$ satisfies the following two fundamental properties:

- $\quad k$-consistency: for all $\mathbf{v}_{h} \in \mathbf{V}_{k}^{h}$ and for all $\mathbf{q} \in\left[\mathbb{P}_{k}(\mathrm{P})\right]^{2}$ it holds

$$
m_{h}^{P}\left(\mathbf{v}_{h}, \mathbf{q}\right)=m^{P}\left(\mathbf{v}_{h}, \mathbf{q}\right)
$$


- stability: there exist two positive constants $\mu_{*}, \mu^{*}$, independent of $h$ and P, such that

$$
\mu_{*} m^{\mathrm{P}}\left(\mathbf{v}_{h}, \mathbf{v}_{h}\right) \leq m_{h}^{P}\left(\mathbf{v}_{h}, \mathbf{v}_{h}\right) \leq \mu^{*} m^{\mathrm{P}}\left(\mathbf{v}_{h}, \mathbf{v}_{h}\right) \quad \forall \mathbf{v}_{h} \in V_{k}^{h} .
$$

The constants $\mu_{*}$ and $\mu^{*}$ may depend on upper and lower bounds of mass density factor $\rho$, cf. (9), and the polynomial degree $k$.

The local bilinear form $a_{h}^{P}$ is given by

$$
a_{h}^{P}\left(\mathbf{v}_{h}, \mathbf{w}_{h}\right)=\int_{\mathrm{P}} \Pi_{k-1}^{0}\left(\varepsilon\left(\mathbf{v}_{h}\right)\right): \Pi_{k-1}^{0}\left(\varepsilon\left(\mathbf{w}_{h}\right)\right) d V+S_{a}^{\mathrm{P}}\left(\mathbf{v}_{h}, \mathbf{w}_{h}\right),
$$

where $S_{a}^{\mathrm{P}}(\cdot, \cdot)$ is the local stabilization term. The bilinear form $a_{h}^{P}$ depends on the orthogonal projections $\Pi_{k-1}^{0} \nabla \mathbf{v}_{h}$ and $\Pi_{k-1}^{0} \nabla \mathbf{w}_{h}$, which are computable from the degrees of freedom of $\mathbf{v}_{h}$ and $\mathbf{w}_{h}$, respectively, see the previous section. $S_{a}^{P}(\cdot, \cdot)$ : $\mathbf{V}_{k}^{h} \times \mathbf{V}_{k}^{h} \rightarrow \mathbb{R}$ and can be any symmetric and coercive bilinear form that is computable from the degrees of freedom and for which there exist two strictly positive real constants $\bar{\sigma}_{*}$ and $\bar{\sigma}^{*}$ such that

$$
\bar{\sigma}_{*} a^{\mathrm{P}}\left(\mathbf{v}_{h}, \mathbf{v}_{h}\right) \leq S_{m}^{\mathrm{P}}\left(\mathbf{v}_{h}, \mathbf{v}_{h}\right) \leq \bar{\sigma}^{*} a^{\mathrm{P}}\left(\mathbf{v}_{h}, \mathbf{v}_{h}\right) \quad \mathbf{v}_{h} \in \operatorname{ker}\left(\Pi_{k}^{0}\right) \cap \mathbf{V}_{k}^{h}(\mathrm{P}) .
$$

Note that $S_{a}^{\mathrm{P}}(\cdot, \cdot)$ must scale like $a^{\mathrm{P}}(\cdot, \cdot)$, that is, as $\mathcal{O}(1)$. We can define computable stabilizations $S_{a}^{\mathrm{P}}(\cdot, \cdot)$ by resorting to the two-dimensional implementations of the effective choices for the scalar case proposed in the literature. ${ }^{47,48}$ The one used in our implementations of the method is discussed in Subsection 3.5.

The discrete bilinear form $a_{h}^{P}(\cdot, \cdot)$ satisfies the two fundamental properties:

- $\quad k$-consistency: for all $\mathbf{v}_{h} \in \mathbf{V}_{k}^{h}$ and for all $\mathbf{q} \in\left[\mathbb{P}_{k}(\mathrm{P})\right]^{2}$ it holds

$$
a_{h}^{P}\left(\mathbf{v}_{h}, \mathbf{q}\right)=a^{\mathrm{P}}\left(\mathbf{v}_{h}, \mathbf{q}\right)
$$

- stability: there exist two positive constants $\alpha_{*}, \alpha^{*}$, independent of $h$ and P, such that

$$
\alpha_{*} a^{\mathrm{P}}\left(\mathbf{v}_{h}, \mathbf{v}_{h}\right) \leq a_{h}^{P}\left(\mathbf{v}_{h}, \mathbf{v}_{h}\right) \leq \alpha^{*} a^{\mathrm{P}}\left(\mathbf{v}_{h}, \mathbf{v}_{h}\right) \quad \forall \mathbf{v}_{h} \in V_{k}^{h} .
$$

The constants $\alpha_{*}$ and $\alpha^{*}$ may depend on the Lamé coefficients $\mu$ and $\lambda$, and the polynomial degree $k{ }^{49}$

Remark 3. We will use the stability of both $m_{h}^{P}(\cdot, \cdot)$ and $a_{h}^{P}(\cdot, \cdot)$ to prove that the semidiscrete virtual element approximation is stable in time, that is, the approximate solution $\mathbf{u}_{h}(t)$ for all $t \in(0, T]$ depends continuously on the initial solutions $\mathbf{u}_{0}$ and $\mathbf{u}_{1}$ and the source term $\mathbf{f}$.

Remark 4. The stability of the symmetric bilinear forms $m_{h}^{P}(\cdot, \cdot)$ and $a_{h}^{P}(\cdot, \cdot)$ implies that both are inner products on $V_{k}^{h}$, and, hence, that they are continuous. In fact, the Cauchy-Schwarz inequality and the local stability of $m_{h}$ imply that

$$
\begin{aligned}
m_{h}\left(\mathbf{v}_{h}, \mathbf{w}_{h}\right) & \leq\left(m_{h}\left(\mathbf{v}_{h}, \mathbf{v}_{h}\right)\right)^{\frac{1}{2}}\left(m_{h}\left(\mathbf{w}_{h}, \mathbf{w}_{h}\right)\right)^{\frac{1}{2}} \leq \mu^{*}\left(m\left(\mathbf{v}_{h}, \mathbf{v}_{h}\right)\right)^{\frac{1}{2}}\left(m\left(\mathbf{w}_{h}, \mathbf{w}_{h}\right)\right)^{\frac{1}{2}} \\
& \leq \mu^{*}\|\rho\|_{\infty}\left\|\mathbf{v}_{h}\right\|_{0}\left\|\mathbf{w}_{h}\right\|_{0}
\end{aligned}
$$

for all $\mathbf{v}_{h} \in \mathbf{V}_{k}^{h}$. Similarly, the Cauchy-Schwarz inequality and the local stability of $a_{h}$ imply that

$$
a_{h}\left(\mathbf{v}_{h}, \mathbf{w}_{h}\right) \leq\left(a_{h}\left(\mathbf{v}_{h}, \mathbf{v}_{h}\right)\right)^{\frac{1}{2}}\left(a_{h}\left(\mathbf{w}_{h}, \mathbf{w}_{h}\right)\right)^{\frac{1}{2}} \leq \alpha^{*}\left(a\left(\mathbf{v}_{h}, \mathbf{v}_{h}\right)\right)^{\frac{1}{2}}\left(a\left(\mathbf{w}_{h}, \mathbf{w}_{h}\right)\right)^{\frac{1}{2}}=\alpha^{*}\left|\mathbf{v}_{h}\right|_{1}\left|\mathbf{w}_{h}\right|_{1}
$$

for all $\mathbf{v}_{h} \in \mathbf{V}_{k}^{h}$.

\section{4 | Approximation of the right-hand side}

We approximate the right-hand side (13) of the semidiscrete formulation (and, consequently, (14) of the full discrete formulation) as follows:

$$
F_{h}\left(\mathbf{v}_{h}\right)=\int_{\Omega} \mathbf{f} \cdot \Pi_{k-2}^{0}\left(\mathbf{v}_{h}\right) d V+\sum_{e \in \Gamma_{N}} \int_{e} \mathbf{g}_{N} \cdot \mathbf{v}_{h} d s \quad \forall \mathbf{v}_{h} \in \mathbf{V}_{k}^{h}
$$


The linear functional $F_{h}(\cdot)$ is clearly computable since $\left.\mathbf{v}_{h}\right|_{e}$ is a polynomial and $\Pi_{k}^{0}\left(\mathbf{v}_{h}\right)$ is computable from the degrees of freedom of $\mathbf{v}_{h}$. Moreover, when $\mathbf{g}_{N}=0$ using the stability of the projection operator and the Cauchy-Schwarz inequality, we note that

$$
\left|F_{h}\left(\mathbf{v}_{h}\right)\right| \leq\left|\int_{\Omega} \mathbf{f}(t) \cdot \Pi_{k-2}^{0}\left(\mathbf{v}_{h}\right) d V\right| \leq\|\mathbf{f}(t)\|_{0}\left\|\Pi_{k-2}^{0}\left(\mathbf{v}_{h}\right)\right\|_{0} \leq\|\mathbf{f}(t)\|_{0}\left\|\mathbf{v}_{h}\right\|_{0} \quad \forall t \in[0, T] .
$$

We will use (35) in the proof of the stability of the semidiscrete virtual element approximation.

\section{5 | The hitchhiker's guide of the VEM for the elastodynamics equation}

In this subsection, we present the implementation details that are practically useful and the basic steps to reduce the implementation of the VEM to the calculation of a few small elemental matrices; cf. also Algorithm 1 where the sketch of the pseudocode is presented. In fact, the implementation of the VEM relies on the $L^{2}$-orthogonal projection matrices for scalar shape functions and their gradients. ${ }^{50}$

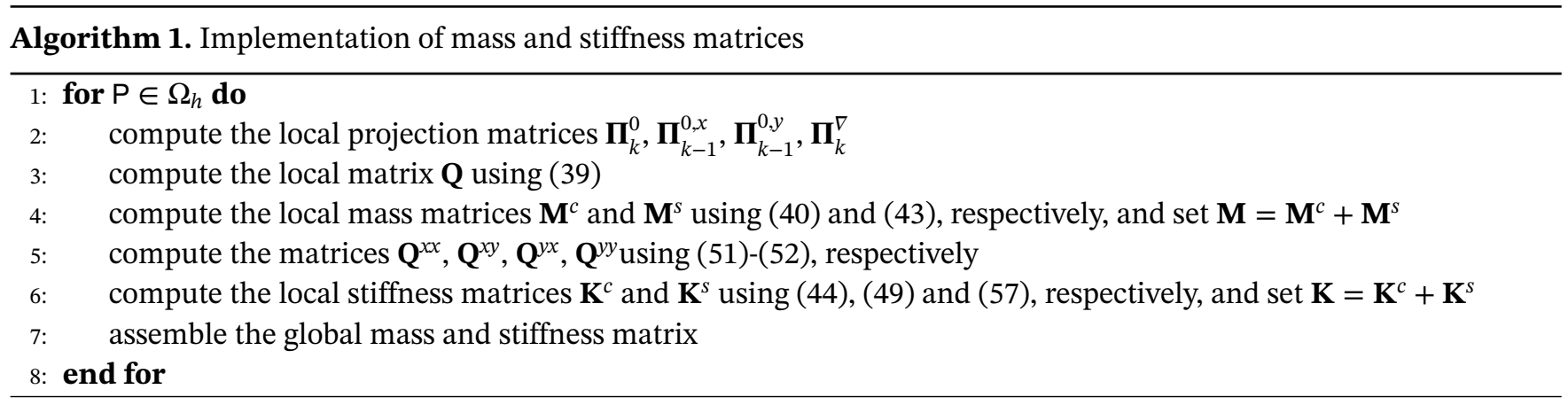

We build the mass and stiffness matrices of the VEM by applying definitions (24) and (28) to the vector-valued shape functions generating $\mathbf{V}_{k}^{h}(\mathrm{P})$. Let $\boldsymbol{\phi}_{i}$ be the $i$ th "canonical" vector-valued basis function of the global virtual element space $\mathbf{V}_{k}^{h}$. We define the mass matrix $\mathbf{M}=\left(\mathrm{M}_{i j}\right)$ and the stiffness matrix $\mathbf{K}=\left(\mathrm{K}_{i j}\right)$ by $\mathrm{M}_{i j}=m_{h}\left(\boldsymbol{\phi}_{j}, \boldsymbol{\phi}_{i}\right)$ and $\mathrm{K}_{i j}=a_{h}\left(\boldsymbol{\phi}_{j}, \boldsymbol{\phi}_{i}\right)$, respectively. The stability condition (27) implies that $\left\|\boldsymbol{\phi}_{i}\right\|_{0}^{2} \lesssim m_{h}\left(\boldsymbol{\phi}_{i}, \boldsymbol{\phi}_{i}\right) \lesssim\left\|\boldsymbol{\phi}_{i}\right\|_{0}^{2}$ for every $i$. Therefore, mass matrix $\mathbf{M}$ is strictly positive definite (and symmetric) and, hence, nonsingular. Similarly, the stability condition (31) implies that $\left|\boldsymbol{\phi}_{i}\right|_{1}^{2} \lesssim a_{h}\left(\boldsymbol{\phi}_{i}, \boldsymbol{\phi}_{i}\right) \lesssim\left|\boldsymbol{\phi}_{i}\right|_{1}^{2}$. Therefore, the stiffness matrix $\mathbf{K}$ is nonnegative definite (and symmetric).

As discussed in the previous subsections, the virtual element space of two-dimensional vector-valued functions $\mathbf{v}_{h} \in \mathbf{V}_{k}^{h}(\mathrm{P})$ is built by taking the two components of $\mathbf{v}_{h}$ in the scalar virtual element space $V_{k}^{h}(\mathrm{P})$. Let $\phi_{i}$ be the shape function of $V_{k}^{h}(\mathrm{P})$ that is associated with the $i$ th degree of freedom, so that by definition, its $i$ th degree of freedom is equal to one, while all other degrees of freedom are equal to zero. Here, we consider the index $i$ (and $j$ in the next formulas) as running from 1 to $N^{\text {dofs }}$, where $N^{\text {dofs }}$ is the dimension of $V_{k}^{h}(\mathrm{P})$. Using this convention, the dimension of the vector virtual element space $\mathbf{V}_{k}^{h}(\mathrm{P})$ is actually $2 N^{\text {dofs }}$. Accordingly, the set of "canonical" shape functions that generate $\mathbf{V}_{k}^{h}(\mathrm{P})$ is given by vector-valued functions of the form $\boldsymbol{\phi}_{i}^{\mathrm{up}}=\left(\phi_{i}, 0\right)^{T}$ and $\phi_{i}^{\text {down }}=\left(0, \phi_{i}\right)^{T}$.

For the exposition's sake, we simplify the notation for the orthogonal projections. More precisely, we use the "hat" symbol over $\phi_{i}$, for example, $\hat{\phi}_{i}$, to denote the $L^{2}$-projection of $\phi_{i}$ onto the polynomials of degree $k$. We also denote the partial derivatives of $\phi_{i}$ along the $x$ and $y$ direction by $\partial_{x} \phi_{i}$ and $\partial_{y} \phi_{i}$, respectively, and, with a small abuse of notation, their $L^{2}$-orthogonal projections onto the polynomials of degree $k-1$ by $\widehat{\partial_{x} \phi_{i}}$ and $\widehat{\partial_{y} \phi_{i}}$ As discussed previously, all these projections are computable from the degrees of freedom of $\phi_{i} \cdot{ }^{50}$

Let $\mathbf{M}=\mathbf{M}^{c}+\mathbf{M}^{s}$ and $\mathbf{K}=\mathbf{K}^{c}+\mathbf{K}^{s}$ be the mass and stiffness matrices, that we write as the sum of the consistence term, that is, matrices $\mathbf{M}^{c}$ and $\mathbf{K}^{c}$, and stability term, that is, matrices $\mathbf{M}^{s}$ and $\mathbf{K}^{s}$. In the rest of this section, we detail the construction of each one of these four matrix terms.

The splitting of the polygonal shape functions in vector-valued functions like $\boldsymbol{\phi}_{i}^{\text {up }}$ and $\boldsymbol{\phi}_{i}^{\text {down }}$, where only one components is actually nonzero, simplifies the expression of the mass matrix significantly. Matrix $\mathbf{M}^{c}$ is, indeed, block diagonal, 
each block has size $N^{\text {dofs }} \times N^{\text {dofs }}$, and its $(i j)$ th entry is given by

$$
\mathbf{M}_{i j}^{c}=\int_{\mathrm{P}} \widehat{\phi}_{i} \widehat{\phi}_{j} d V
$$

Let $\boldsymbol{\Pi}_{k}^{0}$ be the projection matrix of the set of scalar shape functions $\left\{\phi_{i}\right\}_{i=1}^{N^{\text {dofs }}}$. Matrix $\Pi_{k}^{0}$ has size $N^{k} \times N^{\text {dofs }}$, where $N^{k}$ is the dimension of $\mathbb{P}_{k}(\mathrm{P})$. The coefficients of the expansion of $\hat{\phi}_{i}$ on the monomial basis are on the $i$ th columns of $\boldsymbol{\Pi}_{k}^{0}$, so that:

$$
\widehat{\phi}_{i}(x, y)=\sum_{\alpha=1}^{N^{k}} m_{\alpha}(x, y)\left(\mathbf{\Pi}_{k}^{0}\right)_{\alpha, i}
$$

Using this polynomial expansion we find that

$$
\mathbf{M}_{i j}^{c}=\sum_{\alpha, \beta=1}^{N^{k}} \mathbf{Q}_{\alpha, \beta}\left(\boldsymbol{\Pi}_{k}^{0}\right)_{\alpha, i}\left(\boldsymbol{\Pi}_{k}^{0}\right)_{\beta, j}
$$

where $\mathbf{Q}$ is the mass matrix of the monomials,

$$
\mathbf{Q}_{\alpha, \beta}=\int_{\mathrm{P}} m_{\beta}(x, y) m_{\alpha}(x, y) d V .
$$

The equivalent matrix form is

$$
\mathbf{M}^{c}=\left(\boldsymbol{\Pi}_{k}^{0}\right)^{T} \mathbf{Q} \mathbf{\Pi}_{k}^{0} .
$$

The stability matrix used in this work is obtained from the stabilization bilinear form

$$
S_{m}^{\mathrm{P}}\left(\mathbf{v}_{h}, \mathbf{w}_{h}\right)=\bar{\rho} h_{\mathrm{P}}^{2} \sum_{\ell=1}^{2 N^{\mathrm{dofs}}} \operatorname{dof}_{\ell}\left(\mathbf{v}_{h}\right) \operatorname{dof}_{\ell}\left(\mathbf{w}_{h}\right)
$$

where $\bar{\rho}$ is the cell-average of $\rho$ over P. We recall that $\ell$ runs from 1 to $2 N^{\text {dofs }}$ since $N^{\text {dofs }}$ is the number of degrees of freedom of the scalar virtual element space. Using $\phi_{i}^{\text {up }}=\left(\phi_{i}, 0\right)^{T}$ and $\phi_{i}^{\text {down }}=\left(0, \phi_{i}\right)^{T}$ for the vector basis functions, the stability part of the mass matrix is provided by the formula:

$$
\mathbf{M}_{i j}^{s}=\bar{\rho} h_{\mathrm{P}}^{2} \sum_{\ell=1}^{2 N^{\text {dofs }}}\left[\operatorname{dof}_{\ell}\left(\left(1-\Pi_{k}^{0}\right) \boldsymbol{\phi}_{i}^{\text {up }}\right) \operatorname{dof}_{\ell}\left(\left(1-\Pi_{k}^{0}\right) \boldsymbol{\phi}_{j}^{\text {up }}\right)+\operatorname{dof}_{\ell}\left(\left(1-\Pi_{k}^{0}\right) \boldsymbol{\phi}_{i}^{\text {down }}\right) \operatorname{dof}_{\ell}\left(\left(1-\Pi_{k}^{0}\right) \boldsymbol{\phi}_{j}^{\text {down }}\right)\right] .
$$

Using $\phi_{i}^{\text {up }}=\left(\phi_{i}, 0\right)^{T}$ and $\phi_{i}^{\text {down }}=\left(0, \phi_{i}\right)^{T}$ for the vector basis functions, the stability part of the mass matrix is provided by the formula:

$$
\mathbf{M}^{s}=\bar{\rho} h_{\mathrm{P}}^{2}\left[\begin{array}{cc}
\left(\mathbf{I}-\mathbf{\Pi}_{k}^{0}\right)^{T}\left(\mathbf{I}-\mathbf{\Pi}_{k}^{0}\right) & 0 \\
0 & \left(\mathbf{I}-\mathbf{\Pi}_{k}^{0}\right)^{T}\left(\mathbf{I}-\mathbf{\Pi}_{k}^{0}\right)
\end{array}\right] .
$$

The block-diagonal structure above is induced by our choice of using the vector basis functions $\phi_{i}^{\mathrm{up}}=\left(\phi_{i}, 0\right)^{T}$ and $\phi_{i}^{\text {down }}=\left(0, \phi_{i}\right)^{T}$.

The situation is more complex for the stiffness matrix, where the splitting "up - down" induces the $2 \times 2$ splitting:

$$
\mathbf{K}^{c}=\left[\begin{array}{cc}
\mathbf{K}^{\text {c,up,up }} & \mathbf{K}^{\text {c,up,down }} \\
\mathbf{K}^{\text {c,down,up }} & \mathbf{K}^{c, \text { down,down }}
\end{array}\right] .
$$

To detail each one of these four submatrices, consider first a generic vector-valued field $\mathbf{w}=\left(w_{x}, w_{y}\right)^{T}$. From the standard definition of the tensor fields $\varepsilon(\mathbf{w})$ and $\sigma(\mathbf{w})$, we immediately find that:

$$
\varepsilon(\mathbf{w})=\left[\begin{array}{cc}
\partial_{x} w_{x} & \frac{1}{2}\left(\partial_{x} w_{y}+\partial_{y} w_{x}\right) \\
\frac{1}{2}\left(\partial_{x} w_{y}+\partial_{y} w_{x}\right) & \partial_{y} w_{y}
\end{array}\right],
$$


and, according to (8),

$$
\boldsymbol{\sigma}(\mathbf{w})=\left[\begin{array}{cc}
(2 \mu+\lambda) \partial_{x} w_{x}+\lambda \partial_{y} w_{y} & \mu\left(\partial_{x} w_{y}+\partial_{y} w_{x}\right) \\
\mu\left(\partial_{x} w_{y}+\partial_{y} w_{x}\right) & \lambda \partial_{x} w_{x}+(2 \mu+\lambda) \partial_{y} w_{y}
\end{array}\right]
$$

Then, we take $\mathbf{w} \in\left\{\boldsymbol{\phi}_{i}^{\text {up }}, \boldsymbol{\phi}_{i}^{\text {down }}\right\}_{i=1}^{N^{\text {dofs }}}$, so that

$$
\varepsilon\left(\boldsymbol{\phi}_{i}^{\mathrm{up}}\right)=\left[\begin{array}{cc}
\partial_{x} \phi_{i} & \frac{1}{2} \partial_{y} \phi_{i} \\
\frac{1}{2} \partial_{y} \phi_{i} & 0
\end{array}\right], \quad \varepsilon\left(\boldsymbol{\phi}_{i}^{\mathrm{down}}\right)=\left[\begin{array}{cc}
0 & \frac{1}{2} \partial_{x} \phi_{i} \\
\frac{1}{2} \partial_{x} \phi_{i} & \partial_{y} \phi_{i}
\end{array}\right],
$$

and

$$
\boldsymbol{\sigma}\left(\boldsymbol{\phi}_{i}^{\mathrm{up}}\right)=\left[\begin{array}{cc}
(2 \mu+\lambda) \partial_{x} \phi_{i} & \mu \partial_{y} \phi_{i} \\
\mu \partial_{y} \phi_{i} & \lambda \partial_{x} \phi_{i}
\end{array}\right], \quad \boldsymbol{\sigma}\left(\boldsymbol{\phi}_{i}^{\mathrm{down}}\right)=\left[\begin{array}{cc}
\lambda \partial_{y} \phi_{i} & \mu \partial_{x} \phi_{i} \\
\mu \partial_{x} \phi_{i} & (2 \mu+\lambda) \partial_{y} \phi_{i}
\end{array}\right] .
$$

Using such definitions, a straightforward calculation immediately provides us the formulas for the stiffness submatrices:

$$
\begin{aligned}
& \mathbf{K}_{i j}^{\text {c,up,up }}=\int_{\mathrm{P}} \widehat{\sigma\left(\boldsymbol{\phi}_{j}^{\text {up }}\right)}: \widehat{\varepsilon\left(\boldsymbol{\phi}_{i}^{\text {up }}\right)} d V \quad=(2 \mu+\lambda) \int_{\mathrm{P}} \widehat{\partial_{x} \widehat{\phi}_{j}} \widehat{\partial_{x} \phi_{i}} d V+\mu \int_{\mathrm{P}} \widehat{\partial_{y} \phi_{j}} \widehat{\partial_{y} \phi_{i}} d V, \\
& \mathbf{K}_{i j}^{\text {cup,down }}=\int_{\mathrm{P}} \widehat{\sigma\left(\phi_{j}^{\text {up }}\right)}: \varepsilon \widehat{\varepsilon\left(\phi_{i}^{\text {down }}\right)} d V=\mu \int_{\mathrm{P}} \widehat{\partial_{y} \phi_{j}} \widehat{\partial_{x} \phi_{i}} d V+\lambda \int_{\mathrm{P}} \widehat{\partial_{x} \phi_{j}} \widehat{\partial_{y} \phi_{i}} d V \text {, } \\
& \mathbf{K}_{i j}^{c \text { down,up }}=\int_{\mathrm{P}} \boldsymbol{\sigma ( \boldsymbol { \phi } _ { j } ^ { \text { down } } )}: \widehat{\varepsilon\left(\boldsymbol{\phi}_{i}^{\text {up }}\right)} d V=\lambda \int_{\mathrm{P}} \widehat{\partial_{y} \phi_{j}} \widehat{\partial_{x} \phi_{i}} d V+\mu \int_{\mathrm{P}} \widehat{\partial_{x} \phi_{j}} \widehat{\partial_{y} \phi_{i}} d V, \\
& \mathbf{K}_{i j}^{\text {c,down,down }}=\int_{\mathrm{P}} \sigma\left(\widehat{\boldsymbol{\phi}_{j}^{\text {down }}}\right): \varepsilon \widehat{\varepsilon\left(\boldsymbol{\phi}_{i}^{\text {down }}\right)} d V=\mu \int_{\mathrm{P}} \widehat{\partial_{x} \phi_{j}} \widehat{\partial_{x} \phi_{i}} d V+(2 \mu+\lambda) \int_{\mathrm{P}} \widehat{\partial_{y} \phi_{j}} \widehat{\partial_{y} \phi_{i}} d V .
\end{aligned}
$$

A thorough inspection of these formulas reveals that we only need the two projection matrices $\boldsymbol{\Pi}_{k-1}^{0, x}$ and $\boldsymbol{\Pi}_{k-1}^{0, y}$ such that

$$
{\widehat{\partial_{x} \phi_{i}}}_{(x, y)}=\sum_{\alpha=1}^{N^{k-1}} m_{\alpha}(x, y)\left(\boldsymbol{\Pi}_{k-1}^{0, x}\right)_{\alpha, i}, \quad \widehat{\partial}_{y}(x, y)=\sum_{\alpha=1}^{N^{k-1}} m_{\alpha}(x, y)\left(\mathbf{\Pi}_{k-1}^{0, y}\right)_{\alpha, i},
$$

and the four additional matrices involving the derivatives of monomials up to the degree $k$ :

$$
\begin{array}{ll}
\mathbf{Q}_{\alpha, \beta}^{x x}=\int_{\mathrm{P}} \partial_{x} m_{\beta} \partial_{x} m_{\alpha}, d V, & \mathbf{Q}_{\alpha, \beta}^{x y}=\int_{\mathrm{P}} \partial_{x} m_{\beta} \partial_{y} m_{\alpha}, d V, \\
\mathbf{Q}_{\alpha, \beta}^{y x}=\int_{\mathrm{P}} \partial_{y} m_{\beta} \partial_{x} m_{\alpha}, d V, & \mathbf{Q}_{\alpha, \beta}^{y y}=\int_{\mathrm{P}} \partial_{y} m_{\beta} \partial_{x} m_{\alpha}, d V .
\end{array}
$$

Using such matrices we can reformulate the entries of the four subblocks of matrix $\mathbf{K}^{c}$ as follows:

$$
\begin{aligned}
& \mathbf{K}_{i j}^{\text {c,up,up }}=(2 \mu+\lambda) \sum_{\alpha, \beta} \mathbf{Q}_{\alpha, \beta}^{x x}\left(\boldsymbol{\Pi}_{k-1}^{0, x}\right)_{\alpha, i}\left(\boldsymbol{\Pi}_{k-1}^{0, x}\right)_{\beta, j}+\mu \sum_{\alpha, \beta} \mathbf{Q}_{\alpha, \beta}^{y x}\left(\boldsymbol{\Pi}_{k-1}^{0, y}\right)_{\alpha, i}\left(\boldsymbol{\Pi}_{k-1}^{0, y}\right)_{\beta, j}, \\
& \mathbf{K}_{i j}^{c, \text { up,down }}=\mu \sum_{\alpha, \beta} \mathbf{Q}_{\alpha, \beta}^{x y}\left(\boldsymbol{\Pi}_{k-1}^{0, x}\right)_{\alpha, i}\left(\boldsymbol{\Pi}_{k-1}^{0, y}\right)_{\beta, j}+\lambda \sum_{\alpha, \beta} \mathbf{Q}_{\alpha, \beta}^{y x}\left(\boldsymbol{\Pi}_{k-1}^{0, y}\right)_{\alpha, i}\left(\boldsymbol{\Pi}_{k-1}^{0, x}\right)_{\beta, j}, \\
& \mathbf{K}_{i j}^{c, \text { down,up }}=\lambda \sum_{\alpha, \beta} \mathbf{Q}_{\alpha, \beta}^{x y}\left(\boldsymbol{\Pi}_{k-1}^{0, x}\right)_{\alpha, i}\left(\boldsymbol{\Pi}_{k-1}^{0, y}\right)_{\beta, j}+\mu \sum_{\alpha, \beta} \mathbf{Q}_{\alpha, \beta}^{y x}\left(\boldsymbol{\Pi}_{k-1}^{0, y}\right)_{\alpha, i}\left(\boldsymbol{\Pi}_{k-1}^{0, x}\right)_{\beta, j}, \\
& \mathbf{K}_{i j}^{c, \text { down,down }}=\mu \sum_{\alpha, \beta} \mathbf{Q}_{\alpha, \beta}^{x x}\left(\boldsymbol{\Pi}_{k-1}^{0, x}\right)_{\alpha, i}\left(\boldsymbol{\Pi}_{k-1}^{0, x}\right)_{\beta, j}+(2 \mu+\lambda) \sum_{\alpha, \beta} \mathbf{Q}_{\alpha, \beta}^{y y}\left(\boldsymbol{\Pi}_{k-1}^{0, y}\right)_{\alpha, i}\left(\boldsymbol{\Pi}_{k-1}^{0, y}\right)_{\beta, j} .
\end{aligned}
$$

The equivalent compact matrix form is:

$$
\begin{aligned}
\mathbf{K}^{c, \text { up up }} & =(2 \mu+\lambda)\left(\boldsymbol{\Pi}_{k-1}^{0, x}\right)^{T} \mathbf{Q}^{x x} \boldsymbol{\Pi}_{k-1}^{0, x}+\mu\left(\boldsymbol{\Pi}_{k-1}^{0, y}\right)^{T} \mathbf{Q}^{y y} \boldsymbol{\Pi}_{k-1}^{0, y}, \\
\mathbf{K}^{c, \text { up,down }} & =\mu\left(\boldsymbol{\Pi}_{k-1}^{0, x}\right)^{T} \mathbf{Q}^{x y} \boldsymbol{\Pi}_{k-1}^{0, y}+\lambda\left(\boldsymbol{\Pi}_{k-1}^{0, y}\right)^{T} \mathbf{Q}^{y x} \boldsymbol{\Pi}_{k-1}^{0, x}, \\
\mathbf{K}^{c, \text { down,up }} & =\lambda\left(\boldsymbol{\Pi}_{k-1}^{0, x}\right)^{T} \mathbf{Q}^{x y} \boldsymbol{\Pi}_{k-1}^{0, y}+\mu\left(\boldsymbol{\Pi}_{k-1}^{0, y}\right)^{T} \mathbf{Q}^{y x} \boldsymbol{\Pi}_{k-1}^{0, x}, \\
\mathbf{K}^{c, \text { down,down }} & =\mu\left(\boldsymbol{\Pi}_{k-1}^{0, x}\right)^{T} \mathbf{Q}^{x x} \boldsymbol{\Pi}_{k-1}^{0, x}+(2 \mu+\lambda)\left(\boldsymbol{\Pi}_{k-1}^{0, y}\right)^{T} \mathbf{Q}^{y y} \boldsymbol{\Pi}_{k-1}^{0, y} .
\end{aligned}
$$


The stability matrix used in this work is obtained from the stabilization bilinear form

$$
S_{a}^{\mathrm{P}}\left(\mathbf{v}_{h}, \mathbf{w}_{h}\right)=\max (2 \bar{\mu}, \bar{\lambda}) \sum_{\ell=1}^{2 N^{\text {dofs }}} \operatorname{dof}_{\ell}\left(\mathbf{v}_{h}\right) \operatorname{dof}_{\ell}\left(\mathbf{w}_{h}\right)
$$

where $\bar{\mu}$ and $\bar{\lambda}$ are the cell averages of $\mu$ and $\lambda$, respectively. Since we assume that the Lamé coefficients are constant, $\bar{\mu}$ and $\bar{\lambda}$ are respectively equal to $\mu$ and $\lambda$. We recall that index $\ell$ runs from 1 to $2 N^{\text {dofs }}$ because $N^{\text {dofs }}$ is the number of degrees of freedom of the scalar virtual element space. Using $\phi_{i}^{\text {up }}=\left(\phi_{i}, 0\right)^{T}$ and $\phi_{i}^{\text {down }}=\left(0, \phi_{i}\right)^{T}$ for the vector basis functions, the stability part of the mass matrix is provided by the formula:

$$
\mathbf{K}_{i j}^{s}=\max (2 \bar{\mu}, \bar{\lambda}) \sum_{\ell=1}^{N^{\text {dofs }}}\left[\operatorname{dof}_{\ell}\left(\left(1-\Pi_{k}^{\nabla, \mathrm{P}}\right) \boldsymbol{\phi}_{i}^{\text {up }}\right) \operatorname{dof}_{\ell}\left(\left(1-\Pi_{k}^{\nabla, \mathrm{P}}\right) \boldsymbol{\phi}_{j}^{\mathrm{up}}\right)+\operatorname{dof}_{\ell}\left(\left(1-\Pi_{k}^{\nabla, \mathrm{P}}\right) \boldsymbol{\phi}_{i}^{\text {down }}\right) \operatorname{dof}_{\ell}\left(\left(1-\Pi_{k}^{\nabla, \mathrm{P}}\right) \boldsymbol{\phi}_{j}^{\text {down }}\right)\right] .
$$

The stability matrix can be written in block-diagonal form, and in this work we consider

$$
\mathbf{K}^{s}=\max (2 \bar{\mu}, \bar{\lambda})\left[\begin{array}{cc}
\left(\mathbf{I}-\boldsymbol{\Pi}_{k}^{\nabla}\right)^{T}\left(\mathbf{I}-\boldsymbol{\Pi}_{k}^{\nabla}\right) & 0 \\
0 & \left(\mathbf{I}-\boldsymbol{\Pi}_{k}^{\nabla}\right)^{T}\left(\mathbf{I}-\boldsymbol{\Pi}_{k}^{\nabla}\right)
\end{array}\right]
$$

where $\boldsymbol{\Pi}_{k}^{\nabla}$ is the elliptic projection matrix for the scalar case. An alternative formulation can be obtained by considering the orthogonal projector $\Pi_{k}^{0, P}$ instead of the elliptic projector $\Pi_{k}^{\nabla, P}$ in (56), and, consistently, the orthogonal projection matrix $\boldsymbol{\Pi}_{k}^{0}$ instead of $\boldsymbol{\Pi}_{k}^{\nabla}$ in (57).

\section{4 | STABILITY AND CONVERGENCE ANALYSIS FOR THE SEMIDISCRETE PROBLEM}

The main results of this section are stated in Theorems 1,2, and 3, that prove the stability and convergence in the mesh-dependent energy norm that will be introduced in (61) and the convergence in the $L^{2}(\Omega)$-norm, respectively. For exposition's sake, we set $\rho=1$ in (3), (9), and (24) and $\mathbf{g}_{N}=0$ in (5), (11), and (34).

\section{1 | Technicalities and preliminary results}

To carry out the analysis of this section and derive a priori estimates, we need the error estimates for piecewise polynomial approximations and interpolation in the virtual element space $\mathbf{V}_{k}^{h}$ that are stated in the two following lemmas.

Lemma 1. Let $\mathbb{P}_{k}\left(\Omega_{h}\right)$ be the space of piecewise discontinuous polynomials of degree up to $k$ defined on mesh $\Omega_{h}$. Under the mesh regularity assumption (A0), for all $\mathbf{u} \in H^{m+1}(\Omega), m \in \mathbb{N}$, there exists a vector-valued field $\mathbf{u}_{\pi} \in\left[\mathbb{P}_{k}\left(\Omega_{h}\right)\right]^{2}$ such that

$$
\begin{array}{ll}
\left\|\mathbf{u}-\mathbf{u}_{\pi}\right\|_{0} \lesssim \frac{h^{\mu+1}}{k^{m+1}}\|\mathbf{u}\|_{m+1} & \mu=\min (k, m), m \geq 0, \\
\left|\mathbf{u}-\mathbf{u}_{\pi}\right|_{1, h} \lesssim \frac{h^{\mu}}{k^{m}}\|\mathbf{u}\|_{m+1} & \mu=\min (k, m), m \geq 1 .
\end{array}
$$

Proof. The assertion of the lemma is proved in Reference 49.

Lemma 2. Under the mesh regularity assumption (A0), for all $\mathbf{u} \in H^{m+1}(\Omega), m \in \mathbb{N}$, there exists a virtual element interpolant $\mathbf{u}_{I} \in \mathbf{V}_{k}^{h}$ such that

$$
\begin{aligned}
& \left\|\mathbf{u}-\mathbf{u}_{I}\right\|_{0} \lesssim \frac{h^{\mu+1}}{k^{m}}\|\mathbf{u}\|_{m+1} \quad \mu=\min (k, m), m \geq 0, \\
& \left|\mathbf{u}-\mathbf{u}_{I}\right|_{1} \lesssim \frac{h^{\mu}}{k^{m}}\|\mathbf{u}\|_{m+1} \quad \mu=\min (k, m), m \geq 1 .
\end{aligned}
$$


Proof. The assertion of the lemma is proved in Reference 49. See also Reference 51.

Remark 5. The $L^{2}$-estimate provided by Lemma 2 is suboptimal in $k$ as we have a dependence like $k^{m}$ instead of $k^{m+1}$ in the fraction denominators. We point out that, whenever $\Omega$ is convex and the partition $\Omega_{h}$ is made of convex elements, following the proof of proposition 1 in Reference 19 and combining the energy estimate in (59) (where $\mathbf{u}_{I}$ has to be defined as in eq. (20) of Reference 19) with the standard Aubin-Nitsche trick it is possible to prove that the following holds

$$
\left\|\mathbf{u}-\mathbf{u}_{I}\right\|_{0} \lesssim \frac{h^{\mu+1}}{k^{m+1}}\|\mathbf{u}\|_{m+1} \quad \mu=\min (k, m), m \geq 0 .
$$

provided that $h p$ optimal $L^{2}$-error estimates hold for the continuous piecewise polynomial approximation $\varphi_{k}^{h}$ introduced in eq. (4.10) of Reference 52.

\section{2 | Stability}

The semidiscrete virtual element approximation of the time-dependent linear elastodynamics problem in variational form is stable and convergent, cf. Theorems 1 and 2, which are the main results of this section. Moreover, we state Theorems 1 and 2 by using the energy norm

$$
\left\|\left.\left|\mathbf{v}_{h}(t)\left\|\left.\right|^{2}=\right\| \rho^{\frac{1}{2}} \dot{\mathbf{v}}_{h}(t) \|_{0}^{2}+\right| \mathbf{v}_{h}(t)\right|_{1} ^{2}, \quad t \in[0, T]\right.
$$

which is defined for all $v \in \mathbf{V}_{k}^{h}$. The local stability property of the bilinear forms $m_{h}(\cdot, \cdot)$ and $a_{h}(\cdot, \cdot)$ readily imply the equivalence relation

$$
m_{h}\left(\dot{\mathbf{v}}_{h}, \dot{\mathbf{v}}_{h}\right)+a_{h}\left(\mathbf{v}_{h}, \mathbf{v}_{h}\right) \lesssim\left\|\left|\mathbf{v}_{h}(t) \|\right|^{2} \lesssim m_{h}\left(\dot{\mathbf{v}}_{h}, \dot{\mathbf{v}}_{h}\right)+a_{h}\left(\mathbf{v}_{h}, \mathbf{v}_{h}\right)\right.
$$

for all time-dependent virtual element functions $\mathbf{v}_{h}(t)$ with square integrable derivative $\dot{\mathbf{v}}_{h}(t)$.

Remark 6. The hidden constants in (62) are independent of the mesh size parameter $h$. However, they may depend on the stability parameters $\mu_{*}, \mu^{*}, \alpha_{*}, \alpha^{*}$, the regularity constant $\rho$ of the mesh, and the polynomial degree $k .^{52}$ The dependence on $k$ does not seem to have a relevant impact on the optimality of the convergence rates in our numerical experiments in Section 5 .

Theorem 1. Let $\mathbf{f} \in L^{2}\left((0, T] ;\left[L^{2}(\Omega)\right]^{2}\right)$ and let $\mathbf{u}_{h} \in C^{2}\left((0, T] ; \mathbf{V}_{k}^{h}\right)$ be the solution of (13). Then, it holds

$$
\left\|\left|\mathbf { u } _ { h } ( t ) \left\|\left|\lesssim \left\|\left|\left(\mathbf{u}_{0}\right)_{I}\left\|\mid+\int_{0}^{t}\right\| \mathbf{f}(\tau) \|_{0, \Omega} d \tau .\right.\right.\right.\right.\right.\right.
$$

The hidden constant in $\lesssim$ is independent of $h$, but may depend on the model parameters and approximation constants and the polynomial degree $k$.

Proof. We substitute $\mathbf{v}_{h}=\dot{\mathbf{u}}_{h}(t)$ in (13) and, for all $t \in(0, T]$, we obtain

$$
m_{h}\left(\ddot{\mathbf{u}}_{h}, \dot{\mathbf{u}}_{h}\right)+a_{h}\left(\mathbf{u}_{h}, \dot{\mathbf{u}}_{h}\right)=F_{h}\left(\dot{\mathbf{u}}_{h}\right) .
$$

Since both $m_{h}(\cdot, \cdot)$ and $a_{h}(\cdot, \cdot)$ are symmetric bilinear forms, a straightforward calculation yields

$$
\frac{1}{2} \frac{d}{d t}\left(m_{h}\left(\dot{\mathbf{u}}_{h}, \dot{\mathbf{u}}_{h}\right)+a_{h}\left(\mathbf{u}_{h}, \mathbf{u}_{h}\right)\right)=m_{h}\left(\ddot{\mathbf{u}}_{h}, \dot{\mathbf{u}}_{h}\right)+a_{h}\left(\mathbf{u}_{h}, \dot{\mathbf{u}}_{h}\right) .
$$

We substitute this expression in the left-hand side of (64), we integrate in time the resulting equation from 0 to the intermediate time $t$, and using the definition of norm || · ||| in (61) and the equivalence relation (62), we find that

$$
\begin{aligned}
\left\|\mathbf{u}_{h}(t)\right\| \|^{2} & \lesssim m_{h}\left(\dot{\mathbf{u}}_{h}(t), \dot{\mathbf{u}}_{h}(t)\right)+a_{h}\left(\mathbf{u}_{h}(t), \mathbf{u}_{h}(t)\right) \\
& =m_{h}\left(\dot{\mathbf{u}}_{h}(0), \dot{\mathbf{u}}_{h}(0)\right)+a_{h}\left(\mathbf{u}_{h}(0), \mathbf{u}_{h}(0)\right)+2 \int_{0}^{t} F_{h}\left(\dot{\mathbf{u}}_{h}(\tau)\right) d \tau \\
& \lesssim\left\|\mathbf{u}_{h}(0)\right\| \|^{2}+\int_{0}^{t} F_{h}\left(\dot{\mathbf{u}}_{h}(\tau)\right) d \tau .
\end{aligned}
$$


Since $\mathbf{u}_{h}(0)=\left(\mathbf{u}_{0}\right)_{I}$, and using (35) (with $\mathbf{v}_{h}=\dot{\mathbf{u}}_{h}$ ), we find that

$$
\|\| \mathbf{u}_{h}(t)\|\|^{2} \lesssim\left\|\left|\left(\mathbf{u}_{0}\right)_{I}\|\|^{2}+\int_{0}^{t} F_{h}\left(\dot{\mathbf{u}}_{h}(\tau)\right) d \tau \lesssim\left\|\mid\left(\mathbf{u}_{0}\right)_{I}\right\|\left\|^{2}+\int_{0}^{t}\right\| \mathbf{f}(\tau)\left\|_{0}\right\| \dot{\mathbf{u}}_{h}(\tau) \|_{0} d \tau\right.\right.
$$

The thesis follows on applying lemma A5, p. 157 of Reference 53.

\subsection{Convergence analysis in the energy norm}

In this section, we prove the convergence of the semidiscrete virtual element approximation in the energy norm (61). A priori error estimates of the approximation error are derived from Theorem 2 as a corollary, which is reported at the end of the section, by using approximation results for discontinuous polynomial and virtual element spaces.

Theorem 2. Let $\mathbf{u} \in C^{2}\left((0, T] ;\left[H^{m+1}(\Omega)\right]^{2}\right), m \in \mathbb{N}$, be the exact solution of problem (12). Let $\mathbf{u}_{h} \in \mathbf{V}_{k}^{h}$ be the solution of the semidiscrete problem (13) under the mesh regularity assumption (A0). Then, for all $t \in[0, T]$ and all discontinuous polynomial approximations $\mathbf{u}_{\pi}(t)$ of $\mathbf{u}(t)$, it holds that

$$
\left\|\left|\mathbf{u}(t)-\mathbf{u}_{h}(t) \|\right| \lesssim \sup _{\tau \in[0, T]} G_{0}(\tau)+\int_{0}^{t} G_{1}(\tau) d \tau\right.
$$

where

$$
\begin{aligned}
G_{0}(\tau)= & \left\|\dot{\mathbf{u}}(\tau)-\dot{\mathbf{u}}_{I}(\tau)\right\|_{0}+\left|\mathbf{u}(\tau)-\mathbf{u}_{I}(\tau)\right|_{1}+\left|\mathbf{u}(\tau)-\mathbf{u}_{\pi}(\tau)\right|_{1, h}, \\
G_{1}(\tau)= & \left\|\ddot{\mathbf{u}}(\tau)-\ddot{\mathbf{u}}_{I}(\tau)\right\|_{0}+|| \ddot{\mathbf{u}}(\tau)-\ddot{\mathbf{u}}_{\pi}(\tau) \|_{0}+\left|\dot{\mathbf{u}}(\tau)-\dot{\mathbf{u}}_{I}(\tau)\right|_{1}+\left|\dot{\mathbf{u}}(\tau)-\dot{\mathbf{u}}_{\pi}(\tau)\right|_{1, h} \\
& +\sup _{\mathbf{v}_{h} \in \mathbf{V}_{k}^{h} \backslash \mathbb{R}^{2}} \frac{\left|F\left(\mathbf{v}_{h}\right)-F_{h}\left(\mathbf{v}_{h}\right)\right|}{\left|\mathbf{v}_{h}\right|_{1}}
\end{aligned}
$$

The hidden constant in $\lesssim$ is independent of $h$, but may depend on the model parameters, the approximation constants, and the polynomial degree $k$, and the final observation time $T$.

Proof. Since $\mathbf{V}_{k}^{h}$ is a subspace of $\mathbf{V}$, we can take $\mathbf{v}_{h} \in \mathbf{V}_{k}^{h}$ as test function in (12) and subtract from (13) to find the error equation:

$$
m\left(\ddot{\mathbf{u}}(t), \mathbf{v}_{h}\right)-m_{h}\left(\ddot{\mathbf{u}}_{h}(t), \mathbf{v}_{h}\right)+a\left(\mathbf{u}(t), \mathbf{v}_{h}\right)-a_{h}\left(\mathbf{u}_{h}(t), \mathbf{v}_{h}\right)=F\left(\mathbf{v}_{h}\right)-F_{h}\left(\mathbf{v}_{h}\right),
$$

which holds for all $\mathbf{v}_{h} \in \mathbf{V}_{k}^{h}$. Next, we rewrite this equation as $\mathrm{T}_{1}+\mathrm{T}_{2}=\mathrm{T}_{3}$, with the definitions:

$$
\begin{aligned}
\mathrm{T}_{1} & :=m\left(\ddot{\mathbf{u}}, \mathbf{v}_{h}\right)-m_{h}\left(\ddot{\mathbf{u}}_{h}, \mathbf{v}_{h}\right), \\
\mathrm{T}_{2} & :=a\left(\mathbf{u}, \mathbf{v}_{h}\right)-a_{h}\left(\mathbf{u}_{h}, \mathbf{v}_{h}\right), \\
\mathrm{T}_{3} & :=F\left(\mathbf{v}_{h}\right)-F_{h}\left(\mathbf{v}_{h}\right),
\end{aligned}
$$

where we dropped out the explicit dependence on $t$ to simplify the notation. We analyze each term separately. First, we rewrite $\mathrm{T}_{1}$ as

$$
\mathrm{T}_{1}=m_{h}\left(\ddot{\mathbf{u}}_{I}-\ddot{\mathbf{u}}_{h}, \mathbf{v}_{h}\right)+m\left(\ddot{\mathbf{u}}-\ddot{\mathbf{u}}_{\pi}, \mathbf{v}_{h}\right)-m_{h}\left(\ddot{\mathbf{u}}_{I}-\ddot{\mathbf{u}}_{\pi}, \mathbf{v}_{h}\right)
$$

by adding and subtracting $\ddot{\mathbf{u}}_{I}$ and $\ddot{\mathbf{u}}_{\pi}$ to the arguments of $m(\cdot, \cdot)$ and $m_{h}(\cdot, \cdot)$ and noting that $m\left(\ddot{\mathbf{u}}_{\pi}, \mathbf{v}_{h}\right)=m_{h}\left(\ddot{\mathbf{u}}_{\pi}, \mathbf{v}_{h}\right)$ for all $\mathbf{v}_{h} \in \mathbf{V}_{k}^{h}$. We also rewrite $\mathrm{T}_{2}$ as

$$
\mathrm{T}_{2}=a_{h}\left(\mathbf{u}_{I}-\mathbf{u}_{h}, \mathbf{v}_{h}\right)+a\left(\mathbf{u}-\mathbf{u}_{\pi}, \mathbf{v}_{h}\right)-a_{h}\left(\mathbf{u}_{I}-\mathbf{u}_{\pi}, \mathbf{v}_{h}\right)
$$

by adding and subtracting $\mathbf{u}_{I}$ and $\mathbf{u}_{\pi}$ to the arguments of $a(\cdot, \cdot)$ and $a_{h}(\cdot, \cdot)$ and noting that $a\left(\mathbf{u}_{\pi}, \mathbf{v}_{h}\right)=a_{h}\left(\mathbf{u}_{\pi}, \mathbf{v}_{h}\right)$ for all $\mathbf{v}_{h} \in \mathbf{V}_{k}^{h}$. Let $\mathbf{e}_{h}=\mathbf{u}_{I}-\mathbf{u}_{h}$. It holds that $\mathbf{e}_{h}(0)=\dot{\mathbf{e}}_{h}(0)=0$ since $\mathbf{u}_{h}(0)=\left(\mathbf{u}_{0}(0)\right)_{I}=\mathbf{u}_{I}(0)$ and $\dot{\mathbf{u}}_{h}(0)=\left(\mathbf{u}_{1}(0)\right)_{I}=\dot{\mathbf{u}}_{I}(0)$. Then, 
using the definition of $\mathbf{e}_{h}$, we reconsider the error equation

$$
\begin{aligned}
\mathrm{T}_{1}+\mathrm{T}_{2}= & m_{h}\left(\ddot{\mathbf{e}}_{h}, \mathbf{v}_{h}\right)+a_{h}\left(\mathbf{e}_{h}, \mathbf{v}_{h}\right)+m\left(\ddot{\mathbf{u}}-\ddot{\mathbf{u}}_{\pi}, \mathbf{v}_{h}\right)-m_{h}\left(\ddot{\mathbf{u}}_{I}-\ddot{\mathbf{u}}_{\pi}, \mathbf{v}_{h}\right) \\
& +a\left(\mathbf{u}-\mathbf{u}_{\pi}, \mathbf{v}_{h}\right)-a_{h}\left(\mathbf{u}_{I}-\mathbf{u}_{\pi}, \mathbf{v}_{h}\right)=F\left(\mathbf{v}_{h}\right)-F_{h}\left(\mathbf{v}_{h}\right) .
\end{aligned}
$$

Assume that $\mathbf{v}_{h} \neq 0$ and consider the inequalities:

$$
\left|F\left(\mathbf{v}_{h}\right)-F_{h}\left(\mathbf{v}_{h}\right)\right|=\frac{\left|F\left(\mathbf{v}_{h}\right)-F_{h}\left(\mathbf{v}_{h}\right)\right|}{\left|\mathbf{v}_{h}\right|_{1}}\left|\mathbf{v}_{h}\right|_{1} \leq\left(\sup _{\mathbf{v}_{h} \in \mathbf{V}_{k}^{h} \backslash\{0\}} \frac{\left|F\left(\mathbf{v}_{h}\right)-F_{h}\left(\mathbf{v}_{h}\right)\right|}{\left|\mathbf{v}_{h}\right|_{1}}\right)\left|\mathbf{v}_{h}\right|_{1} .
$$

Note that it holds:

$$
\begin{aligned}
& m_{h}\left(\ddot{\mathbf{e}}_{h}, \dot{\mathbf{e}}_{h}\right)+a_{h}\left(\mathbf{e}_{h}, \dot{\mathbf{e}}_{h}\right)=\frac{1}{2} \frac{d}{d t}\left(m_{h}\left(\dot{\mathbf{e}}_{h}, \dot{\mathbf{e}}_{h}\right)+a_{h}\left(\mathbf{e}_{h}, \mathbf{e}_{h}\right)\right), \\
& \left|F\left(\dot{\mathbf{e}}_{h}\right)-F_{h}\left(\dot{\mathbf{e}}_{h}\right)\right| \leq\left(\sup _{\mathbf{v}_{h} \in \mathbf{V}_{k}^{h} \backslash\{0\}} \frac{\left|F\left(\mathbf{v}_{h}\right)-F_{h}\left(\mathbf{v}_{h}\right)\right|}{\left|\mathbf{v}_{h}\right|_{1}}\right)\left\|\left|\mathbf{e}_{h}\right|\right\| .
\end{aligned}
$$

Setting $\mathbf{v}_{h}=\dot{\mathbf{e}}_{h}(t)$ on the left-hand side of (69) and employing (71) and (72) together with (68), we obtain, after rearranging the terms, that:

$$
\begin{aligned}
\frac{1}{2} \frac{d}{d t}\left(m_{h}\left(\dot{\mathbf{e}}_{h}, \dot{\mathbf{e}}_{h}\right)+a_{h}\left(\mathbf{e}_{h}, \mathbf{e}_{h}\right)\right) \leq & -m\left(\ddot{\mathbf{u}}-\ddot{\mathbf{u}}_{\pi}, \dot{\mathbf{e}}_{h}\right)+m_{h}\left(\ddot{\mathbf{u}}_{I}-\ddot{\mathbf{u}}_{\pi}, \dot{\mathbf{e}}_{h}\right)-a\left(\mathbf{u}-\mathbf{u}_{\pi}, \dot{\mathbf{e}}_{h}\right)+a_{h}\left(\mathbf{u}_{I}-\mathbf{u}_{\pi}, \dot{\mathbf{e}}_{h}\right) \\
& +\left(\sup _{\mathbf{v}_{h} \in \mathbf{V}_{k}^{h} \backslash\{0\}} \frac{\left|F\left(\mathbf{v}_{h}\right)-F_{h}\left(\mathbf{v}_{h}\right)\right|}{\left|\mathbf{v}_{h}\right|_{1}}\right)\left\|\mid \mathbf{e}_{h}\right\| \|
\end{aligned}
$$

To ease the notation, we denote the last term above by $\mathrm{R}_{1}(t)$. We integrate in time from 0 to $t$ both sides of (73), note that the initial term is zero since $\mathbf{e}_{h}(0)=\dot{\mathbf{e}}_{h}(0)=0$ and use (62)

$$
\begin{aligned}
\left.\left\|\mathbf{e}_{h}(t)\right\|\right|^{2} \leq & \left.m_{h}\left(\dot{\mathbf{e}}_{h}(t), \dot{\mathbf{e}}_{h}(t)\right)+a_{h}\left(\mathbf{e}_{h}(t), \mathbf{e}_{h}(t)\right)\right) \\
\leq & \int_{0}^{t}\left(\mathrm{R}_{1}(\tau)-m\left(\ddot{\mathbf{u}}(\tau)-\ddot{\mathbf{u}}_{\pi}(\tau), \dot{\mathbf{e}}_{h}(\tau)\right)+m_{h}\left(\ddot{\mathbf{u}}_{I}(\tau)-\ddot{\mathbf{u}}_{\pi}(\tau), \dot{\mathbf{e}}_{h}(\tau)\right)\right. \\
& \left.-a\left(\mathbf{u}(\tau)-\mathbf{u}_{\pi}(\tau), \dot{\mathbf{e}}_{h}(\tau)\right)+a_{h}\left(\mathbf{u}_{I}(\tau)-\mathbf{u}_{\pi}(\tau), \dot{\mathbf{e}}_{h}(\tau)\right)\right) d \tau .
\end{aligned}
$$

Then, we integrate by parts the integral that contains $a(\cdot, \cdot)$ and $a_{h}(\cdot, \cdot)$, and again use the fact that $\mathbf{e}_{h}(0)=\dot{\mathbf{e}}_{h}(0)=0$, to obtain

$$
\begin{aligned}
\left.\left\|\mathbf{e}_{h}(t)\right\|\right|^{2} \leq & \int_{0}^{t}\left(\mathrm{R}_{1}(\tau)+\left[-m\left(\ddot{\mathbf{u}}(\tau)-\ddot{\mathbf{u}}_{\pi}(\tau), \dot{\mathbf{e}}_{h}(\tau)\right)+m_{h}\left(\ddot{\mathbf{u}}_{I}(\tau)-\ddot{\mathbf{u}}_{\pi}(\tau), \dot{\mathbf{e}}_{h}(\tau)\right)\right]\right. \\
& \left.+\left[a\left(\dot{\mathbf{u}}(\tau)-\dot{\mathbf{u}}_{\pi}(\tau), \mathbf{e}_{h}(\tau)\right)-a_{h}\left(\dot{\mathbf{u}}_{I}(\tau)-\dot{\mathbf{u}}_{\pi}(\tau), \mathbf{e}_{h}(\tau)\right)\right]\right) d \tau \\
& +\left[-a\left(\mathbf{u}(t)-\mathbf{u}_{\pi}(t), \mathbf{e}_{h}(t)\right)+a_{h}\left(\mathbf{u}_{I}(t)-\mathbf{u}_{\pi}(t), \mathbf{e}_{h}(t)\right)\right] \\
= & \int_{0}^{t}\left(\mathrm{R}_{1}(\tau)+\mathrm{R}_{2}(\tau)+\mathrm{R}_{3}(\tau)\right) d \tau+\mathrm{R}_{4}(t),
\end{aligned}
$$

where terms $\mathrm{R}_{\ell}, \ell=2,3,4$, match with the squared parenthesis. For the next development, we do not need an upper bound of term $R_{1}$. Instead, we have to bound the other three terms in the right-hand side of (75). To bound $\mathrm{R}_{2}$ we use the continuity of $m(\cdot, \cdot)$ and $m_{h}(\cdot, \cdot)$ and the definition of the energy norm ||$|\cdot|||$ given in (61):

$$
\begin{aligned}
\left|\mathrm{R}_{2}\right| & \leq\left|m\left(\ddot{\mathbf{u}}-\ddot{\mathbf{u}}_{\pi}, \dot{\mathbf{e}}_{h}\right)\right|+\left|m_{h}\left(\ddot{\mathbf{u}}_{I}-\ddot{\mathbf{u}}_{\pi}, \dot{\mathbf{e}}_{h}\right)\right| \lesssim\left(\left\|\ddot{\mathbf{u}}-\ddot{\mathbf{u}}_{\pi}\right\|_{0}+\left\|\ddot{\mathbf{u}}_{I}-\ddot{\mathbf{u}}_{\pi}\right\|_{0}\right)\left\|\dot{\mathbf{e}}_{h}\right\|_{0} \\
& \lesssim\left(\left\|\ddot{\mathbf{u}}-\ddot{\mathbf{u}}_{\pi}\right\|_{0}+\left\|\ddot{\mathbf{u}}_{I}-\ddot{\mathbf{u}}_{\pi}\right\|_{0}\right)\left\||| \mathbf{e}_{h} \mid\right\| .
\end{aligned}
$$


Similarly, to bound $\mathrm{R}_{3}$ we use the continuity of $a(\cdot, \cdot)$ and $a_{h}(\cdot, \cdot)$ and the definition of the energy norm II| $\cdot|| \mid$ given in (61):

$$
\begin{aligned}
\left|\mathrm{R}_{3}\right| & \leq\left|a\left(\dot{\mathbf{u}}-\dot{\mathbf{u}}_{\pi}, \mathbf{e}_{h}\right)\right|+\left|a_{h}\left(\dot{\mathbf{u}}_{I}-\dot{\mathbf{u}}_{\pi}, \mathbf{e}_{h}\right)\right| \lesssim\left(\left|\dot{\mathbf{u}}-\dot{\mathbf{u}}_{\pi}\right|_{1, h}+\left|\dot{\mathbf{u}}_{I}-\dot{\mathbf{u}}_{\pi}\right|_{1, h}\right)\left|\mathbf{e}_{h}\right|_{1} \\
& \lesssim\left(\left|\dot{\mathbf{u}}-\dot{\mathbf{u}}_{\pi}\right|_{1, h}+\left|\dot{\mathbf{u}}_{I}-\dot{\mathbf{u}}_{\pi}\right|_{1, h}\right)\left\|\left|\mathbf{e}_{h}\right|\right\| .
\end{aligned}
$$

Finally, to bound $\mathrm{R}_{4}$ we first use the continuity of $a(\cdot, \cdot)$ and $a_{h}(\cdot, \cdot)$, and the right inequality in (62); then, we apply the Young inequality, so that

$$
\begin{aligned}
\left|\mathrm{R}_{4}\right| & \leq\left|a\left(\mathbf{u}-\mathbf{u}_{\pi}, \mathbf{e}_{h}\right)\right|+\left|a_{h}\left(\mathbf{u}_{I}-\mathbf{u}_{\pi}, \mathbf{e}_{h}\right)\right| \lesssim\left(\left|\mathbf{u}-\mathbf{u}_{\pi}\right|_{1, h}+\left|\mathbf{u}_{I}-\mathbf{u}_{\pi}\right|_{1, h}\right)\left|\mathbf{e}_{h}\right|_{1} \\
& \lesssim\left(\left|\mathbf{u}-\mathbf{u}_{\pi}\right|_{1, h}+\left|\mathbf{u}_{I}-\mathbf{u}_{\pi}\right|_{1, h}\right) \quad||\left|\mathbf{e}_{h}\right||| \lesssim \frac{1}{2 \epsilon}\left(\left|\mathbf{u}-\mathbf{u}_{\pi}\right|_{1, h}+\left|\mathbf{u}_{I}-\mathbf{u}_{\pi}\right|_{1, h}\right)^{2}+\left.\frac{\epsilon}{2}\left|\| \mathbf{e}_{h}\right|\right|^{2} .
\end{aligned}
$$

Using bounds (76), (77), and (78) in (75), we find the inequality

$$
\left\|\left|\mathbf{e}_{h}(t)\right|\right\|^{2} \lesssim \tilde{G}_{0}^{2}(t)+\int_{0}^{t} G_{1}(\tau)\left\|\left|\mathbf{e}_{h}(\tau)\|\| d \tau \lesssim\left(\sup _{\tau \in[0, T]} G_{0}(\tau)\right)^{2}+\int_{0}^{t} G_{1}(\tau)\left\|\mid \mathbf{e}_{h}(\tau)\right\| \| d \tau,\right.\right.
$$

where $\tilde{G}_{0}^{2}(t)=\left(\left|\mathbf{u}(\tau)-\mathbf{u}_{I}(\tau)\right|_{1, h}+\left|\mathbf{u}(\tau)-\mathbf{u}_{\pi}(\tau)\right|_{1, h}\right)^{2}$, and $G_{0}(t)$ and $G_{1}(t)$ are the time-dependent functions defined in (66) and (67). Again, an application of lemma A5, p. 157 of Reference 53 yields

$$
\left\|\mathbf{e}_{h}(t)\right\| \| \sup _{\tau \in[0, T]} G_{0}(\tau)+\int_{0}^{t} G_{1}(\tau) d \tau
$$

The theorem follows on using the triangular inequality

$$
||\left|\mathbf{u}(t)-\mathbf{u}_{h}(t)\right|\left\||\leq||| \mathbf{u}(t)-\mathbf{u}_{I}(t)\right\|||+\left\|\left|\mathbf{u}_{I}(t)-\mathbf{u}_{h}(t)\right|\right\|
$$

and noting that $\left\|\left|\mathbf{u}(t)-\mathbf{u}_{I}(t) \|\right| \mid\right.$ is absorbed in $\sup _{\tau \in[0, T]} G_{0}(\tau)$.

Corollary 1. Under the conditions of Theorem 2, for $\mathbf{f} \in L^{2}\left((0, T) ;\left[H^{m-1}(\Omega)\right]^{2}\right)$ we have that

$$
\begin{aligned}
\sup _{0<t \leq T}\left\|\mathbf{u}(t)-\mathbf{u}_{h}(t)\right\| \lesssim & \frac{h^{\mu}}{k^{m}} \sup _{0<t \leq T}\left(\|\dot{\mathbf{u}}(t)\|_{m+1}+\|\mathbf{u}(t)\|_{m+1}\right) \\
& +\int_{0}^{T}\left(\frac{h^{\mu+1}}{k^{m}}\left(\|\ddot{\mathbf{u}}(\tau)\|_{m+1}+\|\dot{\mathbf{u}}(\tau)\|_{m+1}\right)+\frac{h^{\mu}}{k^{m}}\left(\|\ddot{\mathbf{u}}(\tau)\|_{m+1}+\|\dot{\mathbf{u}}(\tau)\|_{m+1}\right)\right) d \tau \\
& +\int_{0}^{T} h\left\|\left(I-\Pi_{k-2}^{0}\right) \mathbf{f}(\tau)\right\|_{0} d \tau,
\end{aligned}
$$

where $\mu=\min (k, m)$. The hidden constant in " $\lesssim$ " is independent of $h$, but may depend on the model parameters and approximation constants, the polynomial degree $k$, and the final observation time $T$.

Proof. Note that

$$
F_{h}\left(\mathbf{v}_{h}\right)=\int_{\Omega} \mathbf{f} \cdot \Pi_{k-2}^{0} \mathbf{v}_{h} d V=\int_{\Omega} \Pi_{k-2}^{0} \mathbf{f} \cdot \mathbf{v}_{h} d V
$$

For all $\mathbf{v}_{h} \in \mathbf{V}_{k}^{h}$ it holds that

$$
\begin{aligned}
\left|F\left(\mathbf{v}_{h}\right)-F_{h}\left(\mathbf{v}_{h}\right)\right| & =\left|\int_{\Omega}\left(I-\Pi_{k-2}^{0}\right) \mathbf{f} \cdot \mathbf{v}_{h} d V\right|=\left|\int_{\Omega}\left(I-\Pi_{k-2}^{0}\right) \mathbf{f} \cdot\left(I-\Pi_{0}^{0}\right) \mathbf{v}_{h} d V\right| \\
& =\left\|\left.\left(I-\Pi_{k-2}^{0}\right) \mathbf{f}\left|\left\|_{0}\right\|\left(I-\Pi_{0}^{0}\right) \mathbf{v}_{h}\left\|_{0} \lesssim h\right\|\left(I-\Pi_{k-2}^{0}\right) \mathbf{f} \|_{0}\right| \mathbf{v}_{h}\right|_{1} .\right.
\end{aligned}
$$

Hence,

$$
\sup _{\mathbf{v}_{h} \in \mathbf{V}_{k}^{h} \backslash\{0\}} \frac{\left|F\left(\mathbf{v}_{h}\right)-F_{h}\left(\mathbf{v}_{h}\right)\right|}{\left|\mathbf{v}_{h}\right|_{1}} \leq h\left\|\left(I-\Pi_{k-2}^{0}\right) \mathbf{f}\right\|_{0} .
$$


Estimate (79) follows on applying this inequality and the results of Lemmas 1 and 2 to (66) and (67), using the resulting estimates in (65), and taking the supremum on the time interval [0,T].

\subsection{Convergence analysis in the $L^{2}$ norm}

The main result of this section is the following theorem that proves the $L^{2}$-convergence of the conforming VEM. The strategy we use in the proof is inspired by Reference 54, using also the substantial modifications required to set it up in the virtual element framework of Reference 55.

Theorem 3. Let $\mathbf{u}$ be the exact solution of problem (12) under the assumption that domain $\Omega$ is $H^{2}$-regular and $\mathbf{u}_{h} \in$ $\mathbf{V}_{k}^{h}$ the solution of the virtual element method stated in (13) under the mesh assumptions of Section 3.1. If $\mathbf{u}, \dot{\mathbf{u}}, \ddot{\mathbf{u}} \in$ $L^{2}\left(0, T ;\left[H^{m+1}(\Omega) \cap H_{0}^{1}(\Omega)\right]^{2}\right)$, with integer $m \geq 0$, then the following estimate holds for almost every $t \in[0, T]$ by setting $\mu=\min (m, k)$ :

$$
\begin{aligned}
\left\|\mathbf{u}(t)-\mathbf{u}_{h}(t)\right\|_{0} \lesssim & \left\|\mathbf{u}_{h}(0)-\mathbf{u}_{0}\right\|_{0}+\left\|\dot{\mathbf{u}}_{h}(0)-\mathbf{u}_{1}\right\|_{0}+\frac{h^{\mu+1}}{k^{m+1}}\left(\|\ddot{\mathbf{u}}\|_{L^{2}\left(0, T ;\left[H^{m+1}(\Omega)\right]^{2}\right)}\right. \\
& \left.+\|\dot{\mathbf{u}}\|_{L^{2}\left(0, T ;\left[H^{m+1}(\Omega)\right]^{2}\right)}+\|\mathbf{u}\|_{L^{2}\left(0, T ;\left[H^{m+1}(\Omega)\right]^{2}\right)}\right)+\int_{0}^{T}\left\|\left(1-\Pi_{k-2}^{0}\right) \mathbf{f}(\tau)\right\|_{0}^{2} d \tau .
\end{aligned}
$$

The hidden constant in " $\lesssim$ " is independent of $h$, but may depend on the model parameters and approximation constants $\varrho, \mu^{*}$, and the polynomial degree $k$, and the final observation time $T$.

To prove this theorem, we need the energy projection operator $\mathcal{P}_{h}:\left[H_{0}^{1}(\Omega)\right]^{2} \rightarrow \mathbf{V}_{k}^{h}$, which is such that $\mathcal{P}_{h} \mathbf{u} \in \mathbf{V}_{k}^{h}$, for every $\mathbf{u} \in\left[H_{0}^{1}(\Omega)\right]^{2}$, is the solution of the variational problem:

$$
a_{h}\left(\mathcal{P}_{h} \mathbf{u}, \mathbf{v}_{h}\right)=a\left(\mathbf{u}, \mathbf{v}_{h}\right) \quad \forall \mathbf{v}_{h} \in \mathbf{V}_{k}^{h}
$$

The energy projection $\mathcal{P}_{h} \mathbf{u}$ is a virtual element approximation of the exact solution $\mathbf{u}$, and the accuracy of the approximation is characterized by the following lemma.

Lemma 3. Let $\mathbf{u} \in\left[H^{m+1}(\Omega) \cap H_{0}^{1}(\Omega)\right]^{2}$ be the solution of problem (12) under the mesh assumptions of Section 3.1. Then, there exists a unique function $\mathcal{P}_{h} \mathbf{u} \in \mathbf{V}_{k}^{h}$ such that

$$
\left|\mathbf{u}-\mathcal{P}_{h} \mathbf{u}\right|_{1} \lesssim \frac{h^{\mu}}{k^{m}}\|\mathbf{u}\|_{m+1}
$$

with $\mu=\min (k, m)$ and $m \geq 1$. Moreover, if domain $\Omega$ is $H^{2}$-regular, it holds that

$$
\left\|\mathbf{u}-\mathcal{P}_{h} \mathbf{u}\right\|_{0} \lesssim \frac{h^{\mu+1}}{k^{m+1}}\|\mathbf{u}\|_{m+1}
$$

Proof. The proof of this lemma is similar to that of lemma 3.1 in Reference 56 for the conforming virtual element approximation of a scalar parabolic problem and, for this reason, is omitted. The complete proof can be found in Reference 57.

Proof of Theorem 3. We use the energy projection to split the approximation error as follows: $\mathbf{u}(t)-\mathbf{u}_{h}(t)=\rho(t)-\boldsymbol{\eta}(t)$, with $\rho(t)=\mathbf{u}(t)-\mathcal{P}_{h} \mathbf{u}(t)$ and $\boldsymbol{\eta}(t)=\mathbf{u}_{h}(t)-\mathcal{P}_{h} \mathbf{u}(t)$. Since $\mathbf{V}_{k}^{h}$ is a subspace of $\left[H_{0}^{1}(\Omega)\right]^{2}$, to derive the error equation, we test (12) and (13) against $\mathbf{v}_{h} \in \mathbf{V}_{k}^{h}$

$$
\begin{aligned}
& m\left(\ddot{\mathbf{u}}, \mathbf{v}_{h}\right)+a\left(\mathbf{u}, \mathbf{v}_{h}\right)=F\left(\mathbf{v}_{h}\right), \\
& m_{h}\left(\ddot{\mathbf{u}}_{h}, \mathbf{v}_{h}\right)+a_{h}\left(\mathbf{u}_{h}, \mathbf{v}_{h}\right)=F_{h}\left(\mathbf{v}_{h}\right),
\end{aligned}
$$

and take the difference

$$
m_{h}\left(\ddot{\mathbf{u}}_{h}, \mathbf{v}_{h}\right)+a_{h}\left(\mathbf{u}_{h}, \mathbf{v}_{h}\right)-\left(m\left(\ddot{\mathbf{u}}, \mathbf{v}_{h}\right)+a\left(\mathbf{u}, \mathbf{v}_{h}\right)\right)=F_{h}\left(\mathbf{v}_{h}\right)-F\left(\mathbf{v}_{h}\right)
$$


We add and subtract $\mathcal{P}_{h} \ddot{\mathbf{u}}$ and $\mathcal{P}_{h} \mathbf{u}$ in the virtual element bilinear forms $m_{h}$ and $a_{h}$ and rearrange the terms containing $\ddot{\mathbf{u}}$ and $\mathbf{u}$ to the right-hand side to obtain:

$$
\begin{aligned}
m_{h}\left(\ddot{\mathbf{u}}_{h}-\mathcal{P}_{h} \ddot{\mathbf{u}}, \mathbf{v}_{h}\right)+a_{h}\left(\mathbf{u}_{h}-\mathcal{P}_{h} \mathbf{u}, \mathbf{v}_{h}\right)= & F_{h}\left(\mathbf{v}_{h}\right)-F\left(\mathbf{v}_{h}\right)+m\left(\ddot{\mathbf{u}}, \mathbf{v}_{h}\right)+a\left(\mathbf{u}, \mathbf{v}_{h}\right) \\
& -m_{h}\left(\mathcal{P}_{h} \ddot{\mathbf{u}}, \mathbf{v}_{h}\right)-a_{h}\left(\mathcal{P}_{h} \mathbf{u}, \mathbf{v}_{h}\right)
\end{aligned}
$$

Since (84) implies that $a\left(\mathbf{u}, \mathbf{v}_{h}\right)-a_{h}\left(\mathcal{P}_{h} \mathbf{u}, \mathbf{v}_{h}\right)=0$, and using the notation $\ddot{\boldsymbol{\eta}}=\ddot{\mathbf{u}}_{h}-\mathcal{P}_{h} \ddot{\mathbf{u}}$ and $\boldsymbol{\eta}=\mathbf{u}_{h}-\mathcal{P}_{h} \mathbf{u}$, we obtain

$$
m_{h}\left(\ddot{\boldsymbol{\eta}}, \mathbf{v}_{h}\right)+a_{h}\left(\boldsymbol{\eta}, \mathbf{v}_{h}\right)=F_{h}\left(\mathbf{v}_{h}\right)-F\left(\mathbf{v}_{h}\right)+m\left(\ddot{\mathbf{u}}, \mathbf{v}_{h}\right)-m_{h}\left(\mathcal{P}_{h} \ddot{\mathbf{u}}, \mathbf{v}_{h}\right)
$$

Hereafter in this proof, we will assume that

$$
\mathbf{v}_{h}(t)=\int_{t}^{\xi} \boldsymbol{\eta}(\tau) d \tau
$$

for every $t$ and $\xi \in[0, T]$. The function $\mathbf{v}_{h}(t)$ given by (90) obviously belongs to the virtual element space $\mathbf{V}_{k}^{h}$ as it is a linear superposition of virtual element functions in such a space and, thus, can be used as a test function. Since now $\mathbf{v}_{h}$ depends on time $t$, we are allowed to consider its time derivatives. In particular, we observe that the straightforward calculation

$$
\frac{d}{d t}\left(\mathbf{v}_{h} \frac{d}{d t}\left(\mathbf{u}-\mathbf{u}_{h}\right)\right)=\frac{d}{d t}\left(\mathbf{v}_{h} \frac{d}{d t}(\boldsymbol{\rho}-\boldsymbol{\eta})\right)=\frac{d \mathbf{v}_{h}}{d t} \frac{d \rho}{d t}-\frac{d \mathbf{v}_{h}}{d t} \frac{d \boldsymbol{\eta}}{d t}+\mathbf{v}_{h} \frac{d^{2} \boldsymbol{\rho}}{d t^{2}}-\mathbf{v}_{h} \frac{d^{2} \boldsymbol{\eta}}{d t^{2}}
$$

implies the identity

$$
m_{h}\left(\ddot{\boldsymbol{\eta}}, \mathbf{v}_{h}\right)=-m_{h}\left(\dot{\boldsymbol{\eta}}, \dot{\mathbf{v}}_{h}\right)-\frac{d}{d t} m_{h}\left(\dot{\mathbf{u}}-\dot{\mathbf{u}}_{h}, \mathbf{v}_{h}\right)+m_{h}\left(\ddot{\boldsymbol{\rho}}, \mathbf{v}_{h}\right)+m_{h}\left(\dot{\boldsymbol{\rho}}, \dot{\mathbf{v}}_{h}\right)
$$

Therefore, using (91) in (89) yields

$$
\begin{aligned}
-m_{h}\left(\dot{\boldsymbol{\eta}}, \dot{\mathbf{v}}_{h}\right)+a_{h}\left(\boldsymbol{\eta}, \mathbf{v}_{h}\right)= & F_{h}\left(\mathbf{v}_{h}\right)-F\left(\mathbf{v}_{h}\right)+m\left(\ddot{\mathbf{u}}, \mathbf{v}_{h}\right)-m_{h}\left(\mathcal{P}_{h} \ddot{\mathbf{u}}, \mathbf{v}_{h}\right) \\
& +\frac{d}{d t} m_{h}\left(\dot{\mathbf{u}}-\dot{\mathbf{u}}_{h}, \mathbf{v}_{h}\right)-m_{h}\left(\ddot{\boldsymbol{\rho}}, \mathbf{v}_{h}\right)-m_{h}\left(\dot{\boldsymbol{\rho}}, \dot{\mathbf{v}}_{h}\right)
\end{aligned}
$$

We rewrite the approximation error on the source term on the right-hand side of (92) as follows:

$$
F_{h}\left(\mathbf{v}_{h}(t)\right)-F\left(\mathbf{v}_{h}(t)\right)=m\left(\mathbf{f}_{h}(t)-\mathbf{f}(t), \mathbf{v}_{h}(t)\right),
$$

where $\mathbf{f}_{h}(t)=\Pi_{k-2}^{0} \mathbf{f}$ according to (34). Consider the integral quantities ${ }^{55}$

$$
\mathcal{A}_{1}(t)=\int_{0}^{t}\left(\mathbf{f}_{h}(\tau)-\mathbf{f}(\tau)\right) d \tau, \quad \mathcal{A}_{2}(t)=\int_{0}^{t} \ddot{\mathbf{u}}(\tau) d \tau, \quad \mathcal{A}_{3}(t)=\int_{0}^{t} \mathcal{P}_{h} \ddot{\mathbf{u}}(\tau) d \tau
$$

and note that

$$
\begin{gathered}
F_{h}\left(\mathbf{v}_{h}(t)\right)-F\left(\mathbf{v}_{h}(t)\right)=\frac{d}{d t} m\left(\mathcal{A}_{1}, \mathbf{v}_{h}\right)-m\left(\mathcal{A}_{1}, \dot{\mathbf{v}}_{h}\right), \\
m\left(\ddot{\mathbf{u}}, \mathbf{v}_{h}\right)=\frac{d}{d t} m\left(\mathcal{A}_{2}, \mathbf{v}_{h}\right)-m\left(\mathcal{A}_{2}, \dot{\mathbf{v}}_{h}\right), \\
m_{h}\left(\mathcal{P}_{h} \ddot{\mathbf{u}}, \mathbf{v}_{h}\right)=\frac{d}{d t} m_{h}\left(\mathcal{A}_{3}, \mathbf{v}_{h}\right)-m_{h}\left(\mathcal{A}_{3}, \dot{\mathbf{v}}_{h}\right) .
\end{gathered}
$$

Hence, using (95), (96), and (97) in (92) yields

$$
\begin{aligned}
-m_{h}\left(\dot{\boldsymbol{\eta}}, \dot{\mathbf{v}}_{h}\right)+a_{h}\left(\boldsymbol{\eta}, \mathbf{v}_{h}\right)= & \sum_{i=1}^{2}\left[\frac{d}{d t} m\left(\mathcal{A}_{i}, \mathbf{v}_{h}\right)-m\left(\mathcal{A}_{i}, \dot{\mathbf{v}}_{h}\right)\right]-\left[\frac{d}{d t} m_{h}\left(\mathcal{A}_{3}, \mathbf{v}_{h}\right)-m_{h}\left(\mathcal{A}_{3}, \dot{\mathbf{v}}_{h}\right)\right] \\
& +\frac{d}{d t} m_{h}\left(\dot{\mathbf{u}}-\dot{\mathbf{u}}_{h}, \mathbf{v}_{h}\right)-m_{h}\left(\ddot{\boldsymbol{\rho}}, \mathbf{v}_{h}\right)-m_{h}\left(\dot{\boldsymbol{\rho}}, \dot{\mathbf{v}}_{h}\right) .
\end{aligned}
$$


To prove the assertion of the theorem we integrate both sides of (98) with respect to $t$ between 0 and $\xi$; then, we estimate a lower bound for the left-hand side (LHS) and an upper bound for the right-hand side (RHS).

To estimate a lower bound for the left-hand side of (98), we note that $\dot{\mathbf{v}}_{h}=-\boldsymbol{\eta}, \mathbf{v}_{h}(\xi)=0$ and $a_{h}\left(\mathbf{v}_{h}(0), \mathbf{v}_{h}(0)\right) \geq 0$ from the coercivity of $a_{h}$. Thus, we estimate the left-hand side as follows:

$$
\begin{aligned}
\int_{0}^{\xi}[\operatorname{LHS} \text { of Equation (98)] } d t & =\int_{0}^{\xi}\left[m_{h}(\dot{\boldsymbol{\eta}}, \boldsymbol{\eta})-a_{h}\left(\dot{\mathbf{v}}_{h}, \mathbf{v}_{h}\right)\right] d t \\
& =\int_{0}^{\xi} \frac{1}{2} \frac{d}{d t}\left[m_{h}(\boldsymbol{\eta}, \boldsymbol{\eta})-a_{h}\left(\mathbf{v}_{h}, \mathbf{v}_{h}\right)\right] d t \\
& =\frac{1}{2}\left[m_{h}(\boldsymbol{\eta}(\xi), \boldsymbol{\eta}(\xi))-m_{h}(\boldsymbol{\eta}(0), \boldsymbol{\eta}(0))\right]-\frac{1}{2}\left[a_{h}\left(\mathbf{v}_{h}(\xi), \mathbf{v}_{h}(\xi)\right)-a_{h}\left(\mathbf{v}_{h}(0), \mathbf{v}_{h}(0)\right)\right] \\
& \geq \frac{1}{2}\left(m_{h}(\boldsymbol{\eta}(\xi), \boldsymbol{\eta}(\xi))-m_{h}(\boldsymbol{\eta}(0), \boldsymbol{\eta}(0))\right) .
\end{aligned}
$$

To estimate an upper bound for the right-hand side of (98), we note that $\mathcal{A}_{i}(0)=0$ for $i=1,2,3$ and use again the fact $\mathbf{v}_{h}(\xi)=0$. So, for $i=1,2$, a direct integration yield

$$
\begin{aligned}
\int_{0}^{\xi}\left[\frac{d}{d t} m\left(\mathcal{A}_{i}, \mathbf{v}_{h}\right)-m\left(\mathcal{A}_{i}, \dot{\mathbf{v}}_{h}\right)\right] d t & =m\left(\mathcal{A}_{i}(\xi), \mathbf{v}_{h}(\xi)\right)-m\left(\mathcal{A}_{i}(0), \mathbf{v}_{h}(0)\right)-\int_{0}^{\xi} m\left(\mathcal{A}_{i}, \dot{\mathbf{v}}_{h}\right) d t \\
& =-\int_{0}^{\xi} m\left(\mathcal{A}_{i}, \dot{\mathbf{v}}_{h}\right) d t
\end{aligned}
$$

and, similarly,

$$
\begin{aligned}
\int_{0}^{\xi}\left[\frac{d}{d t} m_{h}\left(\mathcal{A}_{3}, \mathbf{v}_{h}\right)-m_{h}\left(\mathcal{A}_{3}, \dot{\mathbf{v}}_{h}\right)\right] d t & =m_{h}\left(\mathcal{A}_{3}(\xi), \mathbf{v}_{h}(\xi)\right)-m_{h}\left(\mathcal{A}_{3}(0), \mathbf{v}_{h}(0)\right)-\int_{0}^{\xi} m_{h}\left(\mathcal{A}_{3}, \dot{\mathbf{v}}_{h}\right) d t \\
& =-\int_{0}^{\xi} m_{h}\left(\mathcal{A}_{3}, \dot{\mathbf{v}}_{h}\right) d t
\end{aligned}
$$

Using (100) and (101) in the right-hand side of (98) and rearranging the terms yield

$$
\begin{aligned}
\int_{0}^{\xi}[\operatorname{RHS} \text { of Equation (98)]dt=} & -\int_{0}^{\xi} m\left(\mathcal{A}_{1}, \dot{\mathbf{v}}_{h}\right) d t+\int_{0}^{\xi}\left(m_{h}\left(\mathcal{A}_{3}, \dot{\mathbf{v}}_{h}\right)-m\left(\mathcal{A}_{2}, \dot{\mathbf{v}}_{h}\right)\right) d t \\
& +\left[m_{h}\left(\dot{\mathbf{u}}(\xi)-\dot{\mathbf{u}}_{h}(\xi), \mathbf{v}_{h}(\xi)\right)-m_{h}\left(\dot{\mathbf{u}}(0)-\dot{\mathbf{u}}_{h}(0), \mathbf{v}_{h}(0)\right)\right] \\
& -\int_{0}^{\xi}\left(m_{h}\left(\ddot{\boldsymbol{\rho}}, \mathbf{v}_{h}\right)+m_{h}\left(\dot{\boldsymbol{\rho}}, \dot{\mathbf{v}}_{h}\right)\right) d t=\mathrm{L}_{1}(\xi)+\mathrm{L}_{2}(\xi)+\mathrm{L}_{3}(\xi)+\mathrm{L}_{4}(\xi) .
\end{aligned}
$$

The proof continues by bounding the four terms $\mathrm{L}_{i}(\xi), i=, 1,2,3,4$, separately.

Estimate of term $L_{1}$. To estimate $\mathrm{L}_{1}$, we first note that $m(\cdot, \cdot)$ is an inner product, so we can apply the Cauchy-Schwarz and the Young inequalities to derive the following bound, which holds for any $t \in[0, \xi]$ :

$$
\left|m\left(\mathcal{A}_{1}(t), \dot{\mathbf{v}}_{h}(t)\right)\right| \leq\left\|\mathcal{A}_{1}(t)\right\|_{0}\left\|\dot{\mathbf{v}}_{h}(t)\right\|_{0} \leq \frac{1}{2}\left\|\mathcal{A}_{1}(t)\right\|_{0}^{2}+\frac{1}{2}\left\|\dot{\mathbf{v}}_{h}(t)\right\|_{0}^{2}
$$

We estimate term $\left\|\mathcal{A}_{1}(t)\right\|_{0}$ by applying Jensen's inequality and Fubini's theorem to exchange the integration order

$$
\begin{aligned}
\left\|\mathcal{A}_{1}(t)\right\|_{0}^{2} & =\int_{\Omega} \mid \int_{0}^{t}\left(\mathbf{f}(\tau)-\left.\Pi_{k-2}^{0}(\mathbf{f}(\tau)) d \tau\right|^{2} d V \leq \int_{\Omega} T\left(\int_{0}^{t}\left|\left(I-\Pi_{k-2}^{0}\right) \mathbf{f}(\tau)\right|^{2} d \tau\right) d V\right. \\
& =T \int_{0}^{t}\left(\int_{\Omega}\left|\left(I-\Pi_{k-2}^{0}\right) \mathbf{f}(\tau)\right|^{2} d V\right) d \tau \leq T \int_{0}^{t}\left\|\left(I-\Pi_{k-2}^{0}\right) \mathbf{f}(\tau)\right\|_{0}^{2} d \tau \\
& \leq T \int_{0}^{T}\left\|\left(I-\Pi_{k-2}^{0}\right) \mathbf{f}(\tau)\right\|_{0}^{2} d \tau
\end{aligned}
$$


We use (103) in (102) and the resulting inequality in the definition of $\mathrm{L}_{1}$; then, we note that the last integral in (103) is on the whole interval $[0, T]$, and is, thus, independent on $t$. Since $\xi \leq T$ and by using the Young's inequality, we readily find that

$$
\begin{aligned}
\left|\mathrm{L}_{1}(\xi)\right| & \leq\left|\int_{0}^{\xi}\left(m\left(\mathcal{A}_{1}(t), \dot{\mathbf{v}}_{h}(t)\right)\right) d t\right| \leq \int_{0}^{\xi}\left|m\left(\mathcal{A}_{1}(t), \dot{\mathbf{v}}_{h}(t)\right)\right| d t \leq \frac{1}{2} \int_{0}^{\xi}\left\|\mathcal{A}_{1}(t)\right\|_{0}^{2} d t+\frac{1}{2} \int_{0}^{\xi}\left\|\dot{\mathbf{v}}_{h}(t)\right\|_{0}^{2} d t \\
& \leq \frac{T^{2}}{2} \int_{0}^{T}\left\|\left(1-\Pi_{k-2}^{0}\right) \mathbf{f}(\tau)\right\|_{0}^{2} d \tau+\frac{1}{2} \int_{0}^{\xi}\left\|\dot{\mathbf{v}}_{h}(t)\right\|_{0}^{2} d t .
\end{aligned}
$$

Estimate of term $L_{2}$. Let $\ddot{\mathbf{u}}_{\pi}$ be any piecewise polynomial approximation of degree at most $k$ of $\ddot{\mathbf{u}}$ that satisfies Lemma 1. Now, consider the piecewise polynomial function defined on mesh $\Omega_{h}$ that is given by

$$
\left(\mathcal{A}_{2}\right)_{\pi}=\int_{0}^{t} \ddot{\mathbf{u}}_{\pi}(\tau) d \tau
$$

Then, we start the estimate of the integral argument of $\mathrm{L}_{2}$ by using consistency property (26) to add and $\operatorname{subtract}\left(\mathcal{A}_{2}\right)_{\pi}$ :

$$
\begin{aligned}
\left|m\left(\mathcal{A}_{2}, \dot{\mathbf{v}}_{h}\right)-m_{h}\left(\mathcal{A}_{3}, \dot{\mathbf{v}}_{h}\right)\right|=\left|m\left(\mathcal{A}_{2}-\left(\mathcal{A}_{2}\right)_{\pi}, \dot{\mathbf{v}}_{h}\right)-m_{h}\left(\mathcal{A}_{3}-\left(\mathcal{A}_{2}\right)_{\pi}, \dot{\mathbf{v}}_{h}\right)\right| \\
\quad=\left|m\left(\mathcal{A}_{2}-\left(\mathcal{A}_{2}\right)_{\pi}, \dot{\mathbf{v}}_{h}\right)-m_{h}\left(\mathcal{A}_{3}-\mathcal{A}_{2}, \dot{\mathbf{v}}_{h}\right)-m_{h}\left(\mathcal{A}_{2}-\left(\mathcal{A}_{2}\right)_{\pi}, \dot{\mathbf{v}}_{h}\right)\right| \\
\quad \leq\left|m\left(\mathcal{A}_{2}-\left(\mathcal{A}_{2}\right)_{\pi}, \dot{\mathbf{v}}_{h}\right)\right|+\left|m_{h}\left(\mathcal{A}_{3}-\mathcal{A}_{2}, \dot{\mathbf{v}}_{h}\right)\right|+\left|m_{h}\left(\mathcal{A}_{2}-\left(\mathcal{A}_{2}\right)_{\pi}, \dot{\mathbf{v}}_{h}\right)\right| \\
\quad \leq\left(\left(1+\mu^{*}\right)\left\|\mathcal{A}_{2}-\left(\mathcal{A}_{2}\right)_{\pi}\right\|_{0}+\mu^{*}\left\|\mathcal{A}_{3}-\mathcal{A}_{2}\right\|_{0}\right)\left\|\dot{\mathbf{v}}_{h}\right\|_{0} \\
\quad \leq\left(1+\mu^{*}\right)\left(\left\|\mathcal{A}_{2}-\left(\mathcal{A}_{2}\right)_{\pi}\right\|_{0}+\left\|\mathcal{A}_{3}-\mathcal{A}_{2}\right\|_{0}\right)\left\|\dot{\mathbf{v}}_{h}\right\|_{0} \\
\quad \leq \frac{\left(1+\mu^{*}\right)}{2}\left(\left\|\mathcal{A}_{2}-\left(\mathcal{A}_{2}\right)_{\pi}\right\|_{0}+\left\|\mathcal{A}_{3}-\mathcal{A}_{2}\right\|_{0}\right)^{2}+\frac{\left(1+\mu^{*}\right)}{2}\left\|\dot{\mathbf{v}}_{h}\right\|_{0}^{2} \\
\quad \leq\left(1+\mu^{*}\right)\left(\left\|\mathcal{A}_{2}-\left(\mathcal{A}_{2}\right)_{\pi}\right\|_{0}^{2}+\left\|\mathcal{A}_{3}-\mathcal{A}_{2}\right\|_{0}^{2}\right)+\frac{\left(1+\mu^{*}\right)}{2}\left\|\dot{\mathbf{v}}_{h}\right\|_{0}^{2} .
\end{aligned}
$$

[add and subtract $\mathcal{A}_{2}$ ]

[use triangular inequality]

[use (32) and continuity of $m$ ] [note that $\mu^{*}<1+\mu^{*}$ ]

[use Young's inequality]

[use $(a+b)^{2} \leq 2 a^{2}+2 b^{2}$ ]

To estimate $\left\|\mathcal{A}_{2}(t)-\left(\mathcal{A}_{2}(t)\right)_{\pi}\right\|_{0}^{2}$, we start $\mathcal{A}_{2}(t)$ and $\left(\mathcal{A}_{2}(t)\right)_{\pi}$ :

$$
\begin{aligned}
& \left\|\mathcal{A}_{2}(t)-\left(\mathcal{A}_{2}(t)\right)_{\pi}\right\|_{0}^{2}=\left\|\int_{0}^{t}\left(\ddot{\mathbf{u}}(\tau)-\ddot{\mathbf{u}}_{\pi}(\tau)\right) d \tau\right\|_{0}^{2} \quad \text { [use definition of } L^{2}(\Omega) \text {-norm] } \\
& =\int_{\Omega}\left|\int_{0}^{t}\left(\ddot{\mathbf{u}}(\tau)-\ddot{\mathbf{u}}_{\pi}(\tau)\right) d \tau\right|^{2} d V \quad \text { [use Jensen's inequality] } \\
& \leq \int_{\Omega}\left(T \int_{0}^{t}\left|\ddot{\mathbf{u}}(\tau)-\ddot{\mathbf{u}}_{\pi}(\tau)\right|^{2} d \tau\right) d V \quad \text { [apply Fubini's Theorem] } \\
& \leq T \int_{0}^{t}\left(\int_{\Omega}\left|\ddot{\mathbf{u}}(\tau)-\ddot{\mathbf{u}}_{\pi}(\tau)\right|^{2} d V\right) d \tau \quad \text { [use definition of } L^{2}(\Omega) \text {-norm] } \\
& =T \int_{0}^{t}\left\|\ddot{\mathbf{u}}(\tau)-\ddot{\mathbf{u}}_{\pi}(\tau)\right\|_{0}^{2} d \tau \quad \text { [apply Lemma 1] } \\
& \lesssim T \frac{h^{2(\mu+1)}}{k^{2(m+1)}} \int_{0}^{t}|\ddot{\mathbf{u}}(\tau)|_{m+1}^{2} d \tau \quad \text { [use definition of } L^{2}\left(0, T ;\left[H^{m+1}(\Omega)\right]^{2}\right) \text {-norm] } \\
& \lesssim T \frac{h^{2(\mu+1)}}{k^{2(m+1)}}\|\ddot{\mathbf{u}}\|_{L^{2}\left(0, T ;\left[H^{m+1}(\Omega)\right]^{2}\right)^{2}}^{2} \text {. }
\end{aligned}
$$

Similarly, to estimate $\left\|\mathcal{A}_{3}(t)-\mathcal{A}_{2}(t)\right\|_{0}^{2}$, we start from the definition of $\mathcal{A}_{3}(t)$ and $\mathcal{A}_{2}(t)$ :

$$
\begin{array}{rlrl}
\left\|\mathcal{A}_{3}(t)-\mathcal{A}_{2}(t)\right\|_{0}^{2}=\left\|\int_{0}^{t}\left(\mathcal{P}_{h} \ddot{\mathbf{u}}(\tau)-\ddot{\mathbf{u}}(\tau)\right) d \tau\right\|_{0}^{2} & & \text { [use definition of } L^{2}(\Omega) \text {-norm] } \\
\quad & =\int_{\Omega}\left|\int_{0}^{t}\left(\mathcal{P}_{h} \ddot{\mathbf{u}}(\tau)-\ddot{\mathbf{u}}(\tau)\right) d \tau\right|^{2} d V & & \text { [use Jensen's inequality] } \\
& \leq \int_{\Omega}\left(T \int_{0}^{t}\left|\mathcal{P}_{h} \ddot{\mathbf{u}}(\tau)-\ddot{\mathbf{u}}(\tau)\right|^{2} d \tau\right) d V & & \text { [apply Fubini's Theorem] } \\
& \leq T \int_{0}^{t}\left(\int_{\Omega}\left|\mathcal{P}_{h} \ddot{\mathbf{u}}(\tau)-\ddot{\mathbf{u}}(\tau)\right|^{2} d V\right) d \tau & & \text { [use definition of } L^{2}(\Omega) \text {-norm] } \\
& =T \int_{0}^{t}\left\|\mathcal{P}_{h} \ddot{\mathbf{u}}(\tau)-\ddot{\mathbf{u}}(\tau)\right\|_{0}^{2} d \tau & & \text { [apply Lemma 3] } \\
\quad \lesssim T \frac{h^{2((\mu+1)}}{k^{2(m+1)}} \int_{0}^{t}|\ddot{\mathbf{u}}(\tau)|_{m+1}^{2} d \tau & & \text { [use definition of } L^{2}\left(0, T ;\left[H^{m+1}(\Omega)\right]^{2}\right) \text {-norm] } \\
& \lesssim T \frac{h^{2(\mu+1)}}{k^{2(m+1)}}\|\ddot{\mathbf{u}}\|_{L^{2}\left(0, T ;\left[H^{m+1}(\Omega)\right]^{2}\right)^{2}}^{2} & &
\end{array}
$$


Using the previous estimates, and noting that the integration on the time interval $[0, \xi], \xi \leq T$, produces an additional factor $T$, we obtain the final upper bound for term $\mathrm{L}_{2}$ :

$$
\left|\mathrm{L}_{2}(\xi)\right| \leq \int_{0}^{\xi}\left|m_{h}\left(\mathcal{A}_{3}, \dot{\mathbf{v}}_{h}\right)-m\left(\mathcal{A}_{2}, \dot{\mathbf{v}}_{h}\right)\right| d t \lesssim\left(1+\mu^{*}\right) T^{2} \frac{h^{2(\mu+1)}}{k^{2(m+1)}}\|\ddot{\mathbf{u}}\|_{L^{2}\left(0, T ;\left(H^{m+1}(\Omega)\right)^{2}\right)}^{2}+\frac{1+\mu^{*}}{2} \int_{0}^{\xi}\left\|\dot{\mathbf{v}}_{h}\right\|_{0}^{2} d t
$$

Estimate of term $L_{3}$. Since $\mathbf{v}_{h}(\xi)=0$ by definition, term $L_{3}$ only depends on the approximation error on $\dot{\mathbf{u}}(0)=\mathbf{u}_{1}$, that is, the initial condition for $\dot{\mathbf{u}}$. Using (32) and Young's inequality yield:

$$
\begin{aligned}
\left|\mathrm{L}_{3}(\xi)\right| & =\left|m_{h}\left(\dot{\mathbf{u}}(0)-\dot{\mathbf{u}}_{h}(0), \mathbf{v}_{h}(0)\right)\right| \leq \mu^{*}\left\|\dot{\mathbf{u}}(0)-\dot{\mathbf{u}}_{h}(0)\right\|_{0}\left\|\mathbf{v}_{h}(0)\right\|_{0} \\
& \leq \frac{\left(\mu^{*}\right)^{2}}{2}\left\|\dot{\mathbf{u}}(0)-\dot{\mathbf{u}}_{h}(0)\right\|_{0}^{2}+\frac{\left(\mu^{*}\right)^{2}}{2}\left\|\mathbf{v}_{h}(0)\right\|_{0}^{2}
\end{aligned}
$$

Estimate of term $L_{4}$. We start from the definition of term $\mathrm{L}_{4}$ and apply the triangular inequality to find that:

$$
\left|\mathrm{L}_{4}(\xi)\right|=\left|\int_{0}^{\xi}\left(m_{h}\left(\ddot{\boldsymbol{\rho}}, \mathbf{v}_{h}\right)+m_{h}\left(\dot{\boldsymbol{\rho}}, \dot{\mathbf{v}}_{h}\right)\right) d t\right| \leq \int_{0}^{\xi}\left|m_{h}\left(\ddot{\boldsymbol{\rho}}, \mathbf{v}_{h}\right)\right| d t+\int_{0}^{\xi}\left|m_{h}\left(\dot{\boldsymbol{\rho}}, \dot{\mathbf{v}}_{h}\right)\right| d t
$$

Both terms on the right depend on the exact solution $\mathbf{u}$ and its approximation provided by the energy projection $\mathcal{P}_{h} \mathbf{u}$. We bound the first term by starting from (32):

$$
\begin{array}{rlrl}
\int_{0}^{\xi} & \left|m_{h}\left(\ddot{\rho}, \mathbf{v}_{h}\right)\right| d t \leq \mu^{*} \int_{0}^{\xi}\|\ddot{\rho}\|\left\|_{0}\right\| \mathbf{v}_{h} \|_{0} d t & & \text { [use Young's inequality] } \\
& \leq \frac{\mu^{*}}{2} \int_{0}^{\xi}\|\ddot{\rho}\|_{0}^{2} d t+\frac{\mu^{*}}{2} \int_{0}^{\xi}\left\|\mathbf{v}_{h}\right\|_{0}^{2} d t & & \text { [apply Lemma 3 to } \ddot{\rho} \text { ] } \\
& \lesssim \frac{\mu^{*}}{2} \frac{h^{2(\mu+1)}}{k^{2(m+1)}} \int_{0}^{\xi}|\ddot{\mathbf{u}}|_{m+1}^{2} d t+\frac{\mu^{*}}{2} \int_{0}^{\xi}\left\|\mathbf{v}_{h}\right\|_{0}^{2} d t & & \text { [use definition of } L^{2}\left(0, T ;\left[H^{m+1}(\Omega)\right]^{2}\right) \text {-norm] } \\
& \lesssim \frac{\mu^{*}}{2} \frac{h^{2(\mu+1)}}{k^{2(m+1)}}\|\ddot{\mathbf{u}}\|_{L^{2}\left(0, T ;\left[H^{m+1}(\Omega)\right]^{2}\right.}^{2}+\frac{\mu^{*}}{2} \int_{0}^{\xi}\left\|\mathbf{v}_{h}\right\|_{0}^{2} d t . &
\end{array}
$$

Similarly, we bound the second term by starting from (32):

$$
\begin{array}{rlrl}
\int_{0}^{\xi} & \left|m_{h}\left(\dot{\boldsymbol{\rho}}, \dot{\mathbf{v}}_{h}\right)\right| d t \leq \mu^{*} \int_{0}^{\xi}\|\dot{\boldsymbol{\rho}}\|_{0}\left\|\dot{\mathbf{v}}_{h}\right\|_{0} d t & & \text { [use Young's inequality] } \\
& \leq \frac{\mu^{*}}{2} \int_{0}^{\xi}\|\dot{\boldsymbol{\rho}}\|_{0}^{2} d t+\frac{\mu^{*}}{2} \int_{0}^{\xi}\left\|\dot{\mathbf{v}}_{h}\right\|_{0}^{2} d t & & \text { [apply Lemma (3) to } \dot{\boldsymbol{\rho}}] \\
& \lesssim \frac{\mu^{*}}{2} \frac{h^{2(\mu+1)}}{k^{2(m+1)}} \int_{0}^{\xi}|\dot{\mathbf{u}}|_{m+1}^{2} d t+\frac{\mu^{*}}{2} \int_{0}^{\xi}\left\|\dot{\mathbf{v}}_{h}\right\|_{0}^{2} d t & & \text { [use definition of } L^{2}\left(0, T ;\left[H^{m+1}(\Omega)\right]^{2}\right) \text {-norm] } \\
& \lesssim \frac{\mu^{*}}{2} \frac{h^{2(\mu+1)}}{k^{2(m+1)}}\|\dot{\mathbf{u}}\|_{L^{2}\left(0, T ;\left[H^{m+1}(\Omega)\right]^{2}\right.}^{2}+\frac{\mu^{*}}{2} \int_{0}^{\xi}\left\|\dot{\mathbf{v}}_{h}\right\|_{0}^{2} d t . &
\end{array}
$$

Using these two estimates in (104), we immediately find that

$$
\left|\mathrm{L}_{4}(\xi)\right| \lesssim \frac{\mu^{*}}{2} \frac{h^{2(\mu+1)}}{k^{2(m+1)}}\left(\|\ddot{\mathbf{u}}\|_{L^{2}\left(0, T ;\left[H^{m+1}(\Omega)\right]^{2}\right.}^{2}+\|\dot{\mathbf{u}}\|_{L^{2}\left(0, T ;\left[H^{m+1}(\Omega)\right]^{2}\right.}^{2}\right)+\mu^{*} \int_{0}^{\xi}\left\|\dot{\mathbf{v}}_{h}\right\|_{0}^{2} d t
$$

Estimate of $\|\boldsymbol{\eta}\|_{0}$ and conclusion of the proof. On collecting the upper bounds of $L_{i}(\xi), i=1,2,3,4$, absorbing the factors $\mu^{*}$ in the hidden constants and using the lower bound (99), we have that

$$
\begin{aligned}
\mu_{*}\|\boldsymbol{\eta}(\xi)\|_{0}^{2}= & \mu_{*} m(\boldsymbol{\eta}(\xi), \boldsymbol{\eta}(\xi)) \leq m_{h}(\boldsymbol{\eta}(\xi), \boldsymbol{\eta}(\xi)) \\
\lesssim & m_{h}(\boldsymbol{\eta}(0), \boldsymbol{\eta}(0))+\left\|\dot{\mathbf{u}}(0)-\dot{\mathbf{u}}_{h}(0)\right\|_{0}^{2}+T^{2} \int_{0}^{T}\left\|\left(I-\Pi_{k-2}^{0}\right) \mathbf{f}(\tau)\right\|_{0}^{2} d \tau \\
& +\frac{h^{2(\mu+1)}}{k^{2(m+1)}}\left(\|\dot{\mathbf{u}}\|_{L^{2}\left(0, T ;\left[H^{m+1}(\Omega)\right]^{2}\right)}^{2}+T^{2}\|\ddot{\mathbf{u}}\|_{L^{2}\left(0, T ;\left[H^{m+1}(\Omega)\right]^{2}\right)}^{2}\right) \\
& +\int_{0}^{\xi}\left\|\dot{\mathbf{v}}_{h}\right\|_{0}^{2} d t+\int_{0}^{\xi}\left\|\mathbf{v}_{h}\right\|_{0}^{2} d t .
\end{aligned}
$$


We estimate the first term in the right-hand side of (106) by using property (26), the definition of $\boldsymbol{\eta}(0)$, adding and subtracting $\mathbf{u}(0)$ and estimate (86):

$$
\begin{aligned}
m_{h}(\boldsymbol{\eta}(0), \boldsymbol{\eta}(0)) & \leq \mu^{*}\|\boldsymbol{\eta}(0)\|_{0}^{2}=\mu^{*}\left\|\mathbf{u}_{h}(0)-\mathcal{P}_{h} \mathbf{u}(0)\right\|_{0}^{2} \\
& \leq 2 \mu^{*}\left\|\mathbf{u}_{h}(0)-\mathbf{u}(0)\right\|_{0}^{2}+2 \mu^{*}\left\|\mathbf{u}(0)-\mathcal{P}_{h} \mathbf{u}(0)\right\|_{0}^{2} \\
& \lesssim\left\|\mathbf{u}_{h}(0)-\mathbf{u}_{0}\right\|_{0}^{2}+\frac{h^{2(\mu+1)}}{k^{2(m+1)}}\left|\mathbf{u}_{0}\right|_{m+1}^{2} .
\end{aligned}
$$

We also estimate the last integral term in (106) by using the definition of the $L^{2}(\Omega)$-norm, definition (90), Jensen's inequality, and Fubini's Theorem to change the integration order:

$$
\begin{aligned}
\left\|\mathbf{v}_{h}(t)\right\|_{0}^{2} & =\int_{\Omega}\left|\int_{t}^{\xi} \boldsymbol{\eta}(\tau) d \tau\right|^{2} d V \leq \int_{\Omega}|\xi-t|\left(\int_{t}^{\xi}|\boldsymbol{\eta}(\tau)|^{2} d \tau\right) d V \\
& =|\xi-t| \int_{t}^{\xi}\left(\int_{\Omega}|\boldsymbol{\eta}(\tau)|^{2} d V\right) d \tau \leq T \int_{0}^{\xi}\|\boldsymbol{\eta}(\tau)\|_{0}^{2} d \tau
\end{aligned}
$$

and

$$
\int_{0}^{\xi}\left\|\mathbf{v}_{h}(t)\right\|_{0}^{2} d t=\left(T \int_{0}^{\xi}\|\boldsymbol{\eta}(\tau)\|_{0}^{2} d \tau\right)\left(\int_{0}^{\xi} d t\right) \leq T^{2} \int_{0}^{\xi}\|\boldsymbol{\eta}(\tau)\|_{0}^{2} d \tau
$$

Using estimates (107) and (109) in (106) and recalling that $\dot{\mathbf{v}}_{h}=-\boldsymbol{\eta}$, we find that

$$
\begin{aligned}
\|\boldsymbol{\eta}(\xi)\|_{0}^{2} \lesssim & \left\|\mathbf{u}(0)-\mathbf{u}_{h}(0)\right\|_{0}^{2}+\left\|\dot{\mathbf{u}}(0)-\dot{\mathbf{u}}_{h}(0)\right\|_{0}^{2}+T^{2} \int_{0}^{T}\left\|\left(I-\Pi_{k-2}^{0}\right) \mathbf{f}(\tau)\right\|_{0}^{2} d \tau \\
& +\frac{h^{2(\mu+1)}}{k^{2(m+1)}}\left(\left|\mathbf{u}_{0}\right|_{m+1}^{2}+\|\dot{\mathbf{u}}\|_{L^{2}\left(0, T ;\left[H^{m+1}(\Omega)\right]^{2}\right.}^{2}+T^{2}\|\ddot{\mathbf{u}}\|_{L^{2}\left(0, T ;\left[H^{m+1}(\Omega)\right]^{2}\right)}^{2}\right) \\
& +\left(1+T^{2}\right) \int_{0}^{\xi}\|\boldsymbol{\eta}(t)\|_{0}^{2} d t .
\end{aligned}
$$

An application of Gronwall's inequality yields, for (almost) every $t \in[0, T]$, the desired upper bound on $\boldsymbol{\eta}(t)$ :

$$
\begin{aligned}
\|\boldsymbol{\eta}(t)\|_{0} \lesssim & C(T)\left(\left\|\mathbf{u}(0)-\mathbf{u}_{h}(0)\right\|_{0}+\left\|\dot{\mathbf{u}}(0)-\dot{\mathbf{u}}_{h}(0)\right\|_{0}+\int_{0}^{T}\left\|\left(1-\Pi_{k-2}^{0}\right) \mathbf{f}(\tau)\right\|_{0} d \tau\right. \\
& \left.+\frac{h^{\mu+1}}{k^{m+1}}\left(\left|\mathbf{u}_{0}\right|_{s+1, \Omega}+\|\dot{\mathbf{u}}\|_{L^{2}\left(0, T ;\left[H^{m+1}(\Omega)\right]^{2}\right.}+\|\ddot{\mathbf{u}}\|_{L^{2}\left(0, T ;\left[H^{m+1}(\Omega]\right)^{2}\right)}\right)\right),
\end{aligned}
$$

where $C(T)$ depends on $T$, the final observation time, and the constant hidden in the " $\lesssim$ " notation depends on $\mu^{*}$ and the mesh regularity constant $\rho$, but is independent of $h$. Finally, we prove the assertion of the theorem through the triangular inequality $\left\|\mathbf{u}_{h}-\mathbf{u}\right\|_{0} \leq\|\rho\|_{0}+\|\boldsymbol{\eta}\|_{0}$ and, then, on using (85) to bound $\rho$ and (111) to bound $\boldsymbol{\eta}$.

\section{5 | NUMERICAL INVESTIGATIONS}

In this section, we carry out number of numerical investigation to validate the VEM by documenting its optimal accuracy and its dissipation/dispersion properties in solving wave propagation problems. To this end, in Section 5.1 we show the optimal convergence properties of the VEM by using a manufactured solution on three different mesh families, each one possessing some special feature. In Section 5.2, we carry out a Von Neumann analysis by studying the dispersion and dissipation of an elementary wave on a periodic domain tessellated in different ways.

Remark 7. All our numerical results are obtained by using the stabilization introduced in Section 3.5. This stabilization is independent of the polynomial degree $k$ and our numerical results are in good accordance with the expected results. 
However, it is known that practical tests in situations like the scalar Poisson problem show that for moderate-to-high values of $k$ this might not be a good choice (especially in three-dimensions). An alternative choice that is surely worth mentioning is provided by the so called "D-recipe" stabilization..$^{47,48,52}$

\section{1 | Accuracy assessment using a manufactured solution}

In this section, we aim to confirm the optimal convergence rate of the numerical approximation of the elastodynamic problem (3)-(7) provided by the virtual element method in accordance with Theorems 2 and 3. In particular, we let $\Omega=(0,1)^{2}$ for $t \in[0, T], T=1$, and consider initial condition $\mathbf{u}_{0}$, boundary condition $\mathbf{g}$ and forcing term $\mathbf{f}$ determined from the exact solution:

$$
\mathbf{u}(x, y, t)=\cos \left(\frac{2 \pi t}{T}\right)\left(\begin{array}{l}
\sin ^{2}(\pi x) \sin (2 \pi y) \\
\sin (2 \pi x) \sin ^{2}(\pi y)
\end{array}\right) .
$$

To this end, we consider three different mesh partitionings, denoted by:

- Mesh 1, randomized quadrilateral mesh;

- Mesh 2, mainly hexagonal mesh with continuously distorted cells;

- Mesh 3, nonconvex octagonal mesh.

The base mesh and the first refined mesh of each mesh sequence are shown in Figure 2.

These mesh sequences have been widely used in the mimetic finite difference and virtual element literature. ${ }^{58}$ The discretization in time is given by applying the leap-frog method with $\Delta t=10^{-4}$ and carried out for $10^{4}$ time cycles in order to reach time $T=1$.

For these calculations, we used the VEM approximation based on the conforming space $V_{k}{ }^{h}$ with $k=1,2,3,4$ and the convergence curves for the three mesh sequences above are reported in Figures 3, 4, and 5. The expected rate of convergence is shown in each panel by the triangle closed to the error curve and indicated by an explicit label. According to Theorem 2, we expect that the approximation error decreases as $\mathcal{O}\left(h^{k}\right)$, or $\mathcal{O}\left(N_{\text {dofs }}^{-k / 2}\right)$ in terms of the number of degrees of freedom that scale like $N_{\text {dofs }} \approx h^{-2}$ when using the virtual element method of order $k$ and measuring the error in the

F I G U R E 2 Base meshes (top row) and first refined meshes (bottom row) of the following mesh families from left to right: randomized quadrilateral mesh; mainly hexagonal mesh; nonconvex octagonal mesh
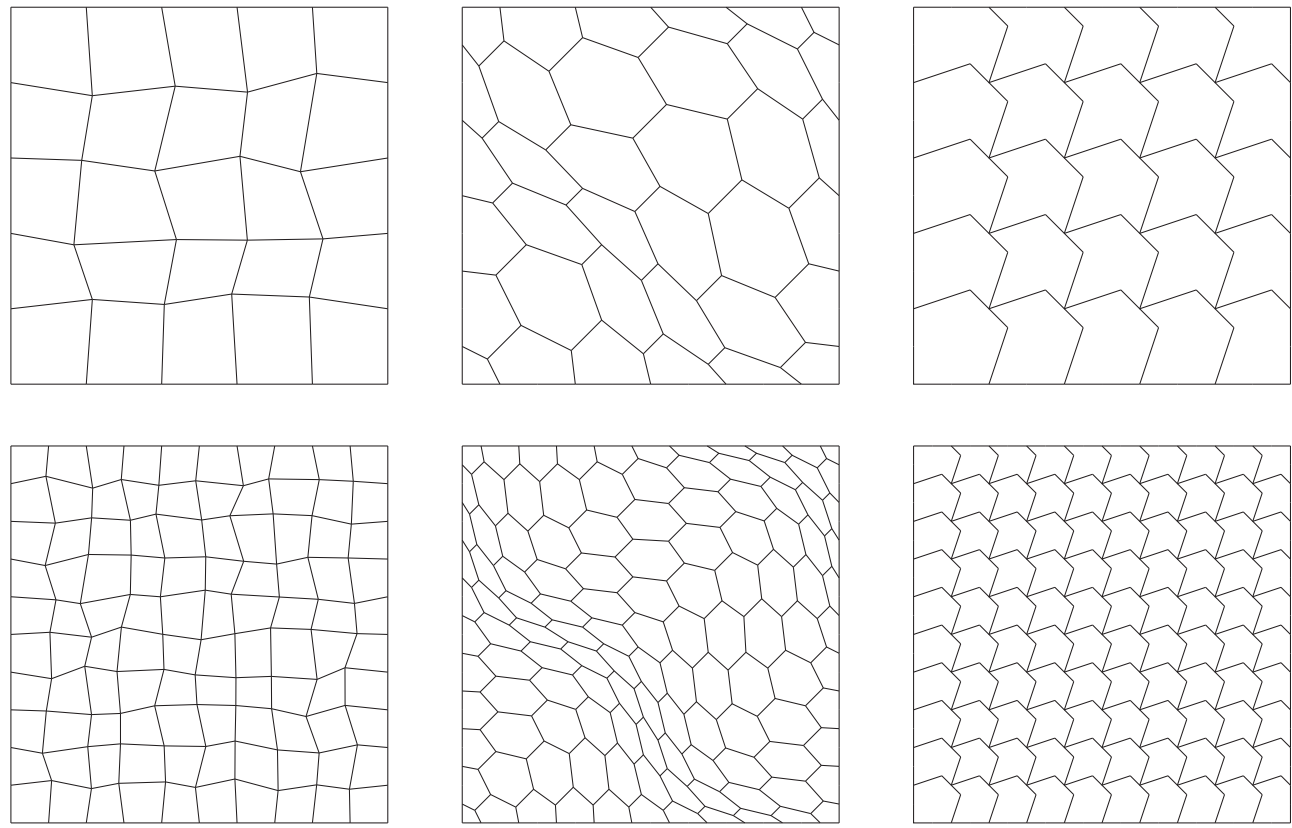

Mesh 1

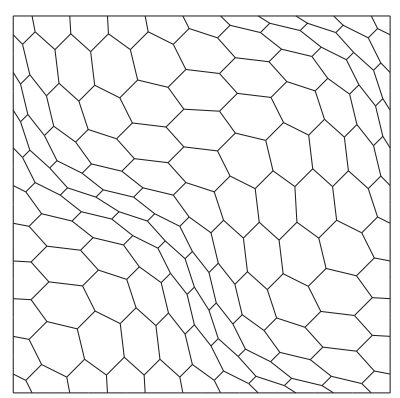

Mesh 2

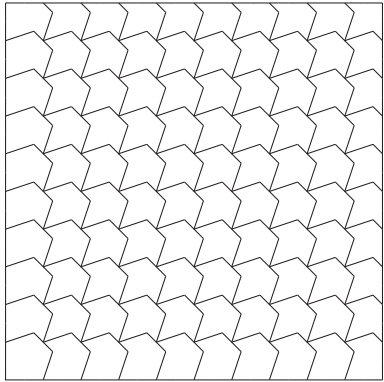

Mesh 3 

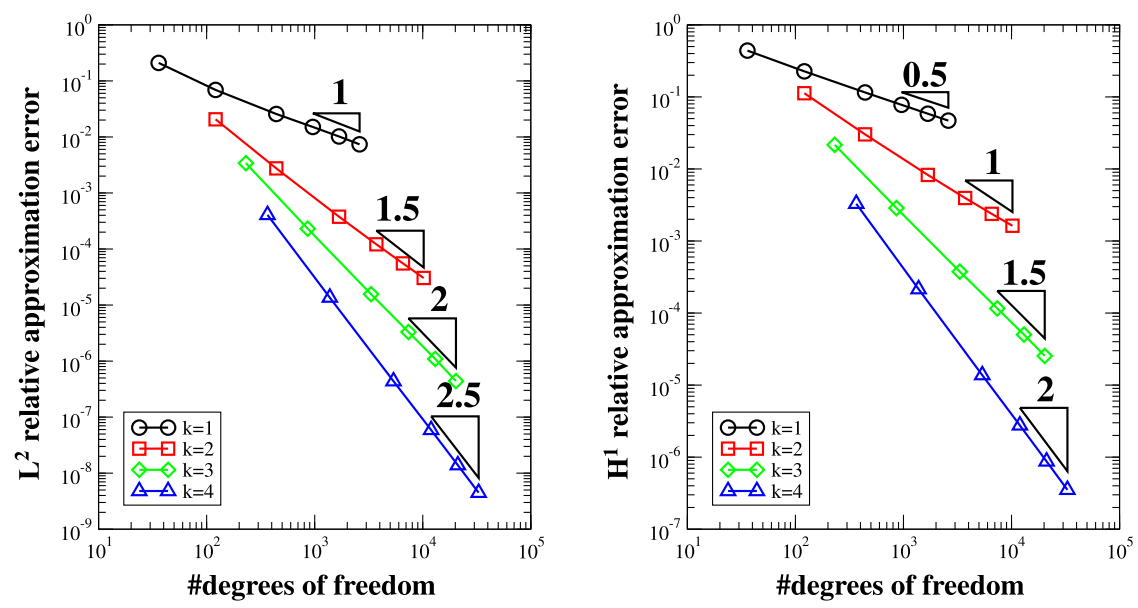

F I G U R E 3 Convergence plots for the virtual element approximation of Problem (3)-(7) with exact solution (112) using family Mesh 1 of randomized quadrilateral meshes. Error curves are computed using the $L^{2}$ norm (left panels) and $H^{1}$ norm (right panels) and are plot versus the number of degrees of freedom
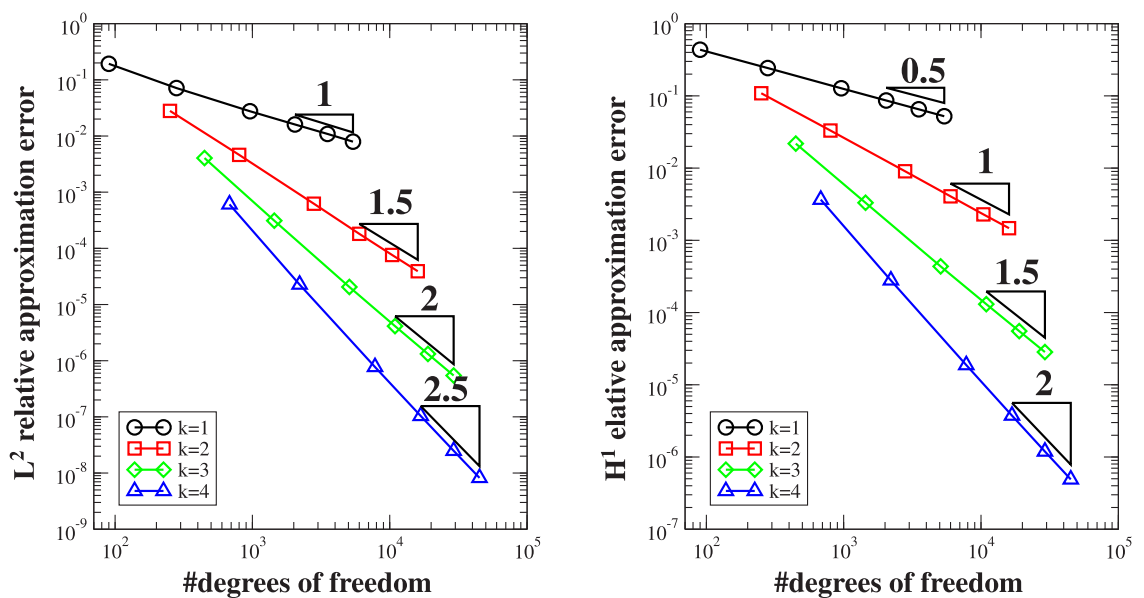

F I G U R E 4 Convergence plots for the virtual element approximation of Problem (3)-(7) with exact solution (112) using family Mesh 2 of mainly hexagonal meshes. Error curves are computed using the $L^{2}$ norm (left panels) and $H^{1}$ norm (right panels) and are plot versus the number of degrees of freedom
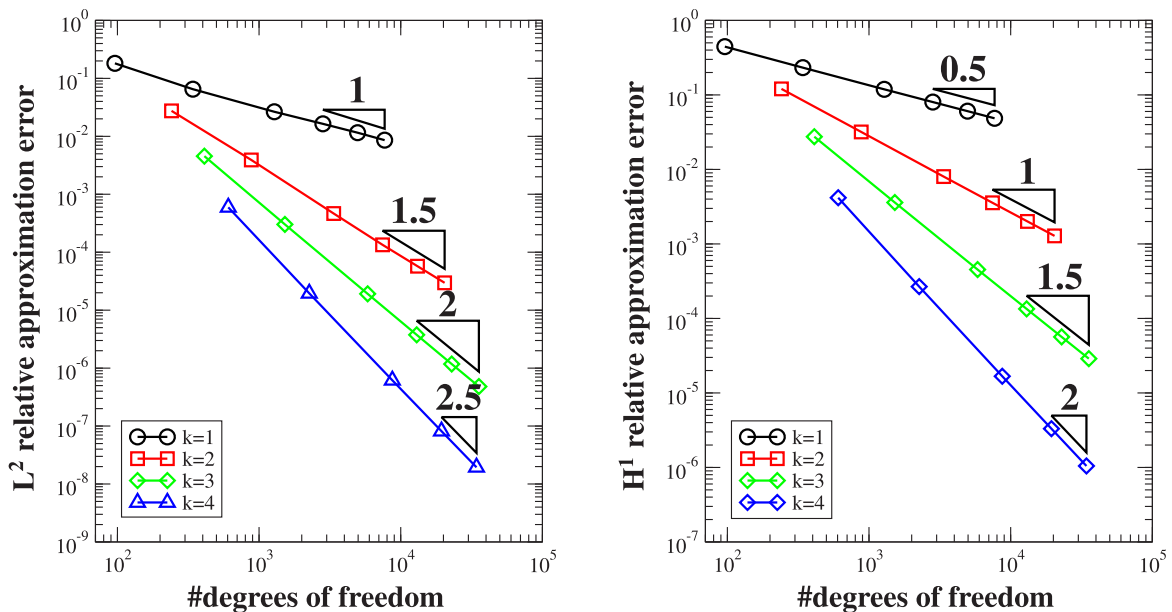

F I G U R E 5 Convergence plots for the virtual element approximation of Problem (3)-(7) with exact solution (112) using family Mesh 3 of nonconvex octagonal meshes. Error curves are computed using the $L^{2}$ norm (left panels) and $H^{1}$ norm (right panels) and are plot versus the number of degrees of freedom

$H^{1}$ norm. Consistently with our approximation, we also expect to see the approximation error to decrease as $\mathcal{O}\left(h^{k+1}\right)$, or $\mathcal{O}\left(N_{\text {dofs }}^{-(k+1) / 2}\right)$, when using the $L^{2}$ norm.

Furthermore, Figure 6 shows the semilog error curves obtained through a "p"-type refinement calculation for the previous benchmark, that is, for a fixed $5 \times 5$ mesh of type $I$ the order of the virtual element space is increased from $k=1$ to $k=10$. We refer the reader to Reference 49 for a detailed presentation of the $p / h p$ virtual element formulations. Here, we compare the performance of the method for two different implementations. In the first one, the space of polynomials of degree $k$ is generated by the standard scaled monomials, while in the second one we consider an orthogonal polynomial 

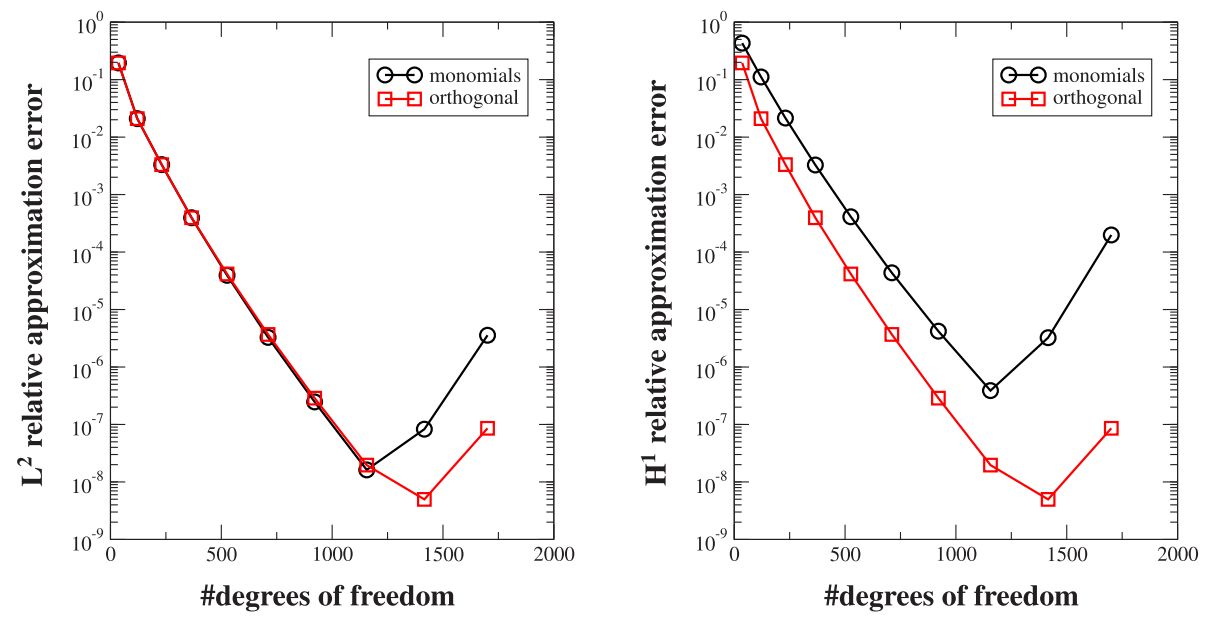

F I G U R E 6 Convergence plots for the virtual element approximation of Problem (3)-(7) with exact solution (112) using family Mesh 1 of randomized quadrilateral meshes. Error curves are computed using k-refinement the $L^{2}$ norm (left panel) and $H^{1}$ norm (right panel) and are plot versus the number of degrees of freedom by performing a refinement of type "p" on a $5 \times 5$ mesh. Each plot shows the two convergence curves that are obtained using monomials (circles) and orthogonalized polynomials (squares)

basis. The impact that such a basis can have on the accuracy of the high-order VEM has already been shown in the VEM literature. ${ }^{47,48,59}$ The behavior of the VEM when using nonorthogonal and orthogonal polynomials basis shown in Figure 6 is in accordance with the literature.

These plots confirm that the conforming VEM formulations proposed in this work provide a numerical approximation with optimal convergence rate on a set of representative mesh sequences, including deformed and nonconvex polygonal cells.

\section{2 | Dispersion and dissipation analysis}

In this section we want to study the dispersion and dissipation errors for the VEM presented before, by means of the Von Neumann analysis, that is, the propagation of a plane wave in a homogeneous medium. For a plane wave, we recall that the numerical dispersion is the gap between the phase of the numerical solution and that of the exact solution while numerical dissipation represents a decrease in the amplitude.

In order to carry out the dispersion and dissipation analysis, we make some standard hypothesis for the following plane wave analysis. ${ }^{60-62}$ Indeed, we suppose that the medium is isotropic, homogeneous, unbounded, and source-free. The latter assumptions are, in general, not respected when considering realistic geophysical applications. However, this analysis provides important information about the discretization parameters to be used in practice for the numerical simulation.

Consider Equation (3) for an unbounded elastic medium where no external forces are applied and seek for solutions in the following form ${ }^{63}$

$$
\mathbf{u}(\mathbf{x}, t)=\mathbf{a} e^{i(\mathbf{k} \cdot \mathbf{x}-\omega t)},
$$

where $\mathbf{a}=\left[a_{1}, a_{2}\right]^{T}$ is the wave amplitude, $\omega$ is the angular frequency, and $\mathbf{k}=2 \pi / L(\cos \theta, \sin \theta)$ is the wavenumber vector, with $L$ being the wavelength and $\theta$ being the angle between the wave propagation direction and the coordinate axes. Then, by taking the real part of (113) the physical wave is recovered. Under these conditions the semidiscrete problem (13) becomes

$$
\mathrm{MÜ}+\mathrm{KU}=\mathbf{0},
$$

where $\mathrm{M}$ and $\mathrm{K}$ are defined as in Section 3.5. To comply with the conditions of periodicity and unboundedness, we solve problem (114) over the domain $E_{r e f}$ with uniform size $h$, and impose periodic boundary conditions on its boundary, see 


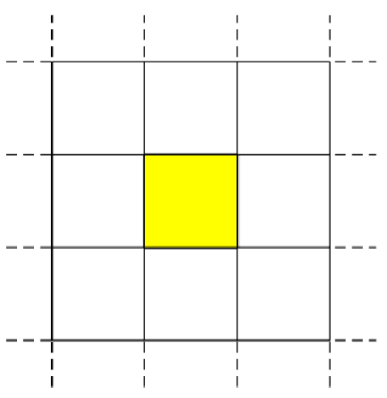

(A) Quadrilateral grid

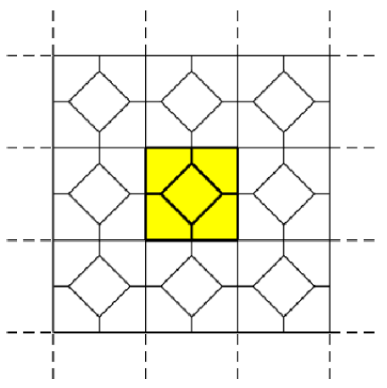

(D) C2 grid

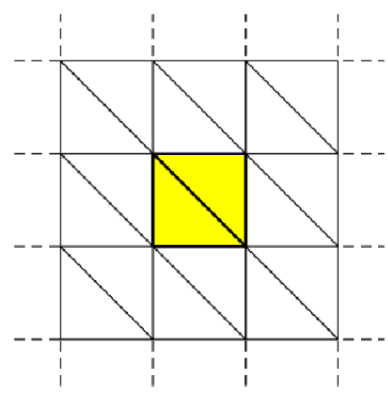

(B) Triangular grid

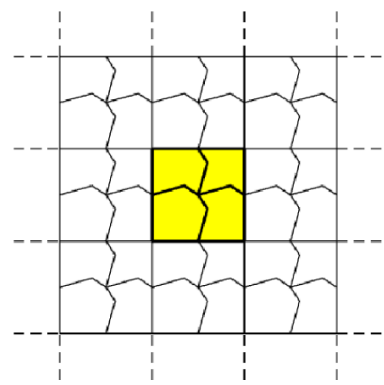

(E) C3 grid

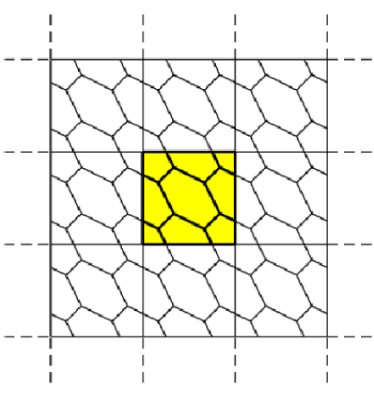

(C) $\mathrm{C} 1$ grid

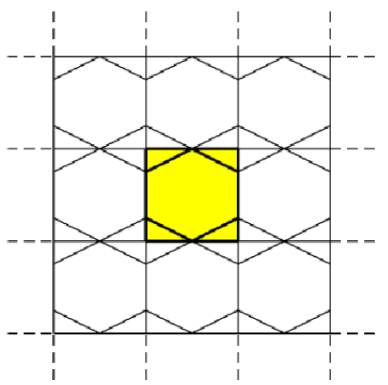

(F) C4 grid

F I G U R E 7 Periodic reference element $E_{\text {ref }}$ (yellow) and periodic reference grids. Quadrilateral (A), triangular (B), and composite $\mathrm{C} 1-\mathrm{C} 4(\mathrm{C}-\mathrm{F})$ grids

Figure 7. The periodic reference domain $E_{\text {ref }}$ can be $(a)$ a square; $(b)$ the union of two triangles; $(c)$ the union of a set of polygons, namely, four hexagons, two quadrilaterals, and two pentagons; $(d)$ the union of one square and four pentagons; $(e)$ four octagons; $(f)$ one hexagon and four triangles.

To apply periodic boundary conditions on $E_{\text {ref }}$, we define the set of "master" and "slave" indexes, respectively, denoted by filled and empty symbols in Figure 8 for the case of the virtual elements with polynomial degree $k=2$. Master indexes refer to the degrees of freedom in which the solution is computed and slave indexes to those where the periodicity conditions are imposed. Since the solution is a plane wave (113), we impose periodic boundary conditions through a suitable projection matrix $\mathrm{P}$ and obtain the system ${ }^{5,7,64,65}$

$$
\mathcal{M U}+\mathcal{K} \mathbf{U}=\mathbf{0},
$$

where $\mathcal{K}$ and $\mathcal{M}$ are given respectively by $\mathcal{K}=\mathrm{P}^{T} \mathrm{~K} P$ and $\mathcal{M}=\mathrm{P}^{T} \mathrm{M} P$.

Substitute $\mathrm{T}^{7,65,66}$ (113) into (115), discretize the system by means of the leap-frog scheme (14) and obtain a generalized eigenvalue problem of the form

$$
\mathrm{KU}_{0}=\Lambda \mathrm{MU}_{0}
$$

where the eigenvalues $\Lambda$ can be expressed in term of the numerical angular frequency $\omega$ through the relation

$$
\Lambda=\frac{4}{\Delta t^{2}} \sin ^{2}\left(\omega \frac{\Delta t}{2}\right)
$$

We use this equation after solving the eigenvalue problem (116) in order to derive the grid-dispersion relations as it will be shown later on. We remark that for two-dimensional elastic wave propagation only two eigenvalues in (116) have a physical meaning as they are related to $\mathrm{P}$ and $\mathrm{S}$ waves. ${ }^{67}$ All the other eigenvalues correspond to nonphysical modes. ${ }^{60}$ Therefore, the relative dispersion errors are given by

$$
e_{P}=\frac{c_{P, h}}{c_{P}}-1, \quad e_{S}=\frac{c_{S, h}}{c_{S}}-1
$$




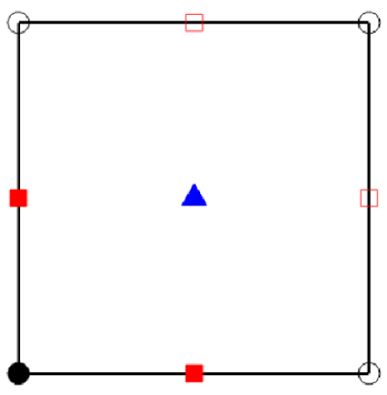

(A) Quadrilateral grid

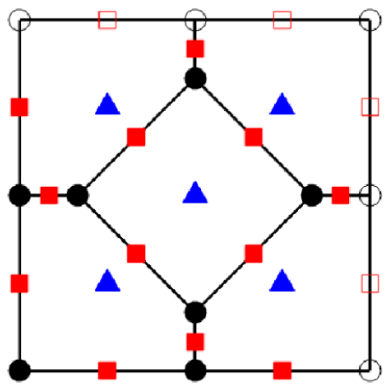

(D) C2 grid

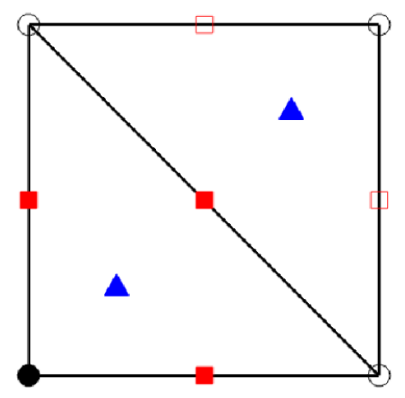

(B) Triangular grid

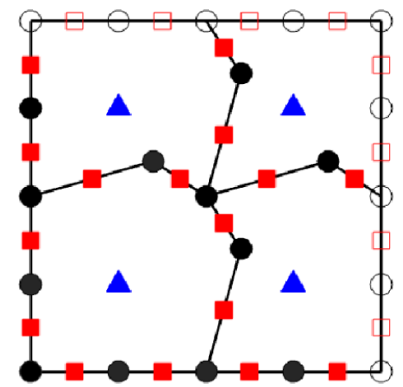

(E) C3 grid

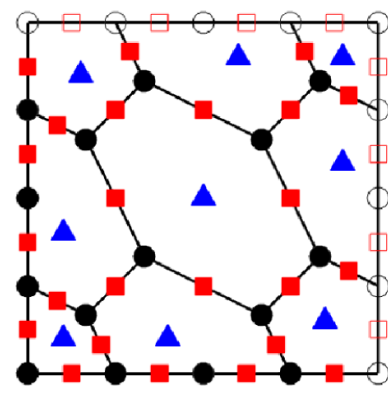

(C) C1 grid

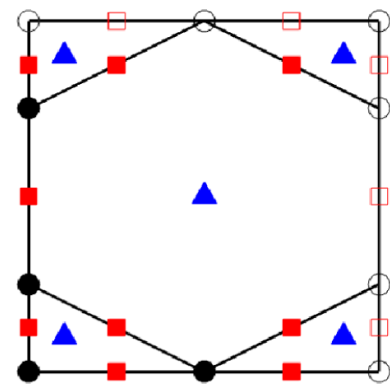

(F) $\mathrm{C} 4$ grid

F I G U R E 8 Degrees of freedom on the reference element $E_{\text {ref }}$ of the local virtual element space with polynomial degree $k=2$. Quadrilateral (A), triangular (B), and composite (C-F) grids: where periodic boundary conditions are assigned, the degrees of freedom are represented by empty squares or circles

where the numerical wave velocities $c_{P, h}$ and $c_{S, h}$ are therefore given by

$$
c_{P, h}=\frac{h \omega_{P, h}}{2 \pi \delta r}, \quad c_{S, h}=\frac{h \omega_{S, h}}{2 \pi \delta}
$$

where $\delta=h /(k L)$ represents the sampling ratio, that is, $\delta^{-1}$ is the number of interpolation points per wavelength, and $L$ is the wavelength.

Now, we study the stability properties of the leap-frog time-stepping scheme and then we analyze the dispersion errors introduced by the numerical formulation. Since the leap-frog method is an explicit time integrator, the time step $\Delta t$ must satisfy the Courant, Friedrichs, Lewy (CFL) condition

$$
\Delta t \leq C_{C F L} \frac{h}{c_{P}}
$$

where we recall that $h$ is the meshsize and $c_{P}$ is the P-wave velocity and we need to impose that $C_{C F L} \leq 1$. We are interested in studying how the $C_{C F L}$ factor may depend on the physical parameters considered in the model, that is, $\lambda$ and $\mu$, and the polynomial degree $k$.

With this aim, we consider (116) and rewrite matrices $\mathrm{K}$ and $\mathrm{M}$ by using a scaling argument, so that

$$
\tilde{\mathbf{K}} \mathbf{U}_{0}=\Lambda^{\prime} \tilde{\mathbf{M}} \mathbf{U}_{0}
$$

where $\Lambda^{\prime}=(h / \Delta t)^{2} \sin ^{2}\left(\omega_{h} \Delta t / 2\right)$. Then, we introduce the stability parameter $q=c_{P} \frac{\Delta t}{h}$ for which it holds $q^{2} \Lambda^{\prime}=$ $c_{P}^{2} \sin ^{2}\left(\frac{\omega_{h} \Delta t}{2}\right) \leq c_{P}^{2}$, that is equivalent to

$$
q \leq \frac{c_{P}}{\sqrt{\Lambda^{\prime}}}=C_{C F L}\left(\Lambda^{\prime}\right) .
$$


The eigenvalue $\Lambda^{\prime}$ depends on the wavenumber vector $\mathbf{k}$ and, therefore, on the values of the angle $\theta .^{68}$ Thus, condition (120) is equivalent to

$$
q \leq c^{*}(\lambda, \mu) \frac{1}{\sqrt{\Lambda_{\max }^{\prime}}}=q_{C F L}
$$

where $\Lambda_{\max }^{\prime}$ is the maximum eigenvalue of (119), taken with respect to the value of $\theta$.

Constant $c^{*}$ depends on the Lamé parameters $\lambda$ and $\mu .^{69}$

We notice that a different approach (that does not make use of a plane wave analysis) is employed in Park et al. (2019) ${ }^{10}$ to estimate $q_{C F L}$ for VEM approximation on arbitrary mesh. The authors were there interested in establishing a rule of thumb for choosing the discretization parameters that satisfy (121), here the analysis is mainly focused on the dispersion and dissipation property of the scheme, provided that condition (121) holds true. Finally, we remark that the present analysis is valid for mesh configurations that have a periodic pattern. For a general polygonal mesh, made by elements of arbitrary shape, one should consider the associated covering mesh made by rectangular elements and check that, for the latter grid, a sufficient number of points per wavelength is employed. This can be inferred easily by applying the rule of thumb that will be given for quadrilateral elements.

\subsection{1 | Numerical dispersion and dissipation analysis: Space discretization}

We start the section by analyzing the dispersion and dissipation properties of the VEM by assuming exact time integration. For this case, we consider Equation (115) and compute analytically the second time derivative of the displacement vector. In this way, we can obtain a generalized eigenvalue problem whose eigenvalues $\Lambda$ can be written in term of the angular frequency $\omega$ through the formula $\Lambda=\omega^{2}$. Grid dispersion errors are computed according to (117): (i) we solve the generalized eigenvalue problem numerically and obtain the eigenvalues $\xi=\omega_{h}^{2}$ that represent the best approximations of the angular frequencies of the traveling waves; (ii) we identify the numerical eigenvalues $\xi_{P}$ and $\xi_{S}$, that correspond to the physical frequencies of the longitudinal and transversal displacement; (iii) we compute the numerical angular frequencies $\omega_{P, h}=\sqrt{\xi_{P}}$ and $\omega_{S, h}=\sqrt{\xi_{S}}$ for the P-wave and the S-wave, respectively.

We now study the dispersion errors as a function of the polynomial degree $k$, the sampling ratio $\delta$ for the shortest wavelength, the wavenumber vector $\mathbf{k}$ of the plane wave, that is, the angle $\theta$, and the ratio $r=c_{P} / c_{S}$. In this first test case, we set $\delta=0.2$, that is, five nodes per shortest wavelength, $\theta=\pi / 4$ and $r=2$. Different choices of the angle $\theta$ provide similar results as it is discussed later on. For all the considered grids, an exponential decay of the dispersion error with respect to the polynomial degree $k$ can be observed, cf. Figure 9. In particular for $k \geq 4$ the dispersion errors are below the threshold value $10^{-6}$ for all the considered grids, with the composite grid $\mathrm{C} 1$ providing the best results.

However, if we look at the same results as a function of total number of degrees of freedom in Figure 10 we see that the quadrilateral and triangular meshes outperform the other grid configurations. This result is important from the computational point of view and should be taken into account for realistic applications. For completeness, in Table 1 we show the dispersion errors for $r=2,5,10$ computed on the quadrilateral and triangular grid. Note that the same exponential decay of the dispersion error is observed for $r=5$ and $r=10$. Moreover, on such grid and in the variation range of $r$, the dispersion errors are negligible, that is, less than $10^{-6}$, when we use a polynomial approximation degree $k>4$. We obtained a similar behavior on the other grids (C1-C4), but we do not report the results here for the sake of brevity.

In Figures 11, 12, and 13, we report the computed dispersion versus $\delta$ for different approximation degrees $k=2,3,4$ and $r=2$. Similar results can be obtained for $r=5,10$ but we do not report them for the sake of brevity. By computing the slopes of the $e_{S}$-and $e_{P}$-curves, we can obtain the following empirical estimate of the orders of convergence: $\left|e_{P}\right|=\left|e_{S}\right|=\mathcal{O}\left(h^{2 k}\right)$ for the VEM with odd polynomial degrees and $\left|e_{P}\right|=\left|e_{S}\right|=\mathcal{O}\left(h^{2 k-1}\right)$ for the VEM with even polynomial degree. These results are in agreement with previous quantitative estimates quadrilateral elements. ${ }^{64,70}$ From the reported result we can infer that with a polynomial degree $k=4$ and $\delta=0.2$, that is, five points per shortest wavelength, the dispersion error is lower than the threshold $10^{-6}$ for all mesh elements (see Figure 13).

Finally, we study the dispersion errors as a function of the angle $\theta$ of the plane wave. In Figure 14 we show the results obtained with the virtual element discretization for polynomial degree $k=4$, the sampling ratio $\delta=0.5$ and $r=2$. Anisotropy ratios $c_{P, h} / c_{P}$ and $c_{S, h} / c_{S}$ have been computed with the same set of values, see Figure 14 . Notice that with quadrilateral and $\mathrm{C} 4$ grids the error is symmetric with respect to the origin of the axes, whereas with triangular grids 

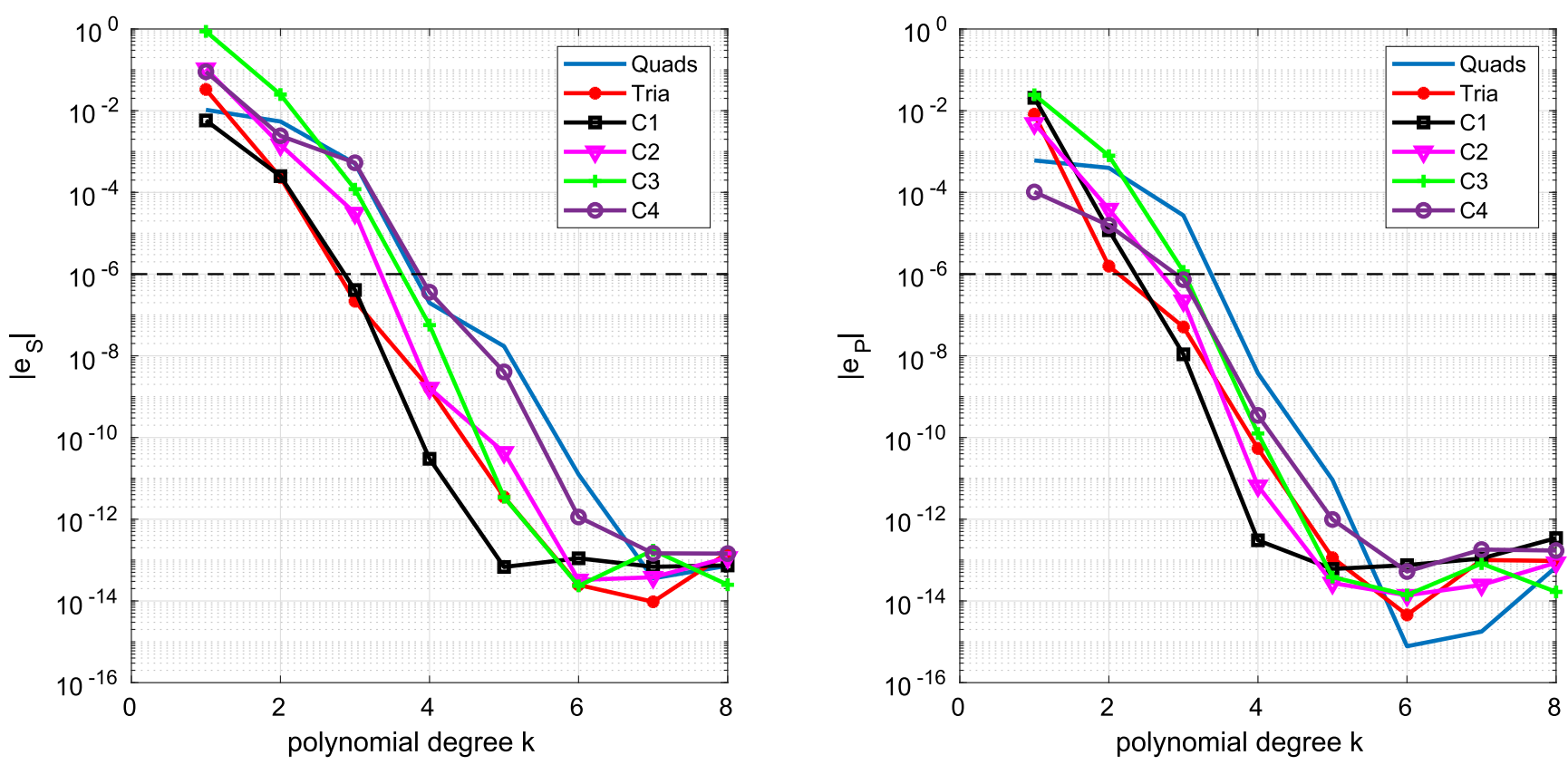

F I G U R E 9 Computed dispersion errors $\left|e_{S}\right|$ and $\left|e_{P}\right|$ as a function of $k$ for $\delta=0.2, \theta=\pi / 4, r=2$ and different mesh configuration. The threshold value $10^{-6}$ is also reported with a black dotted line
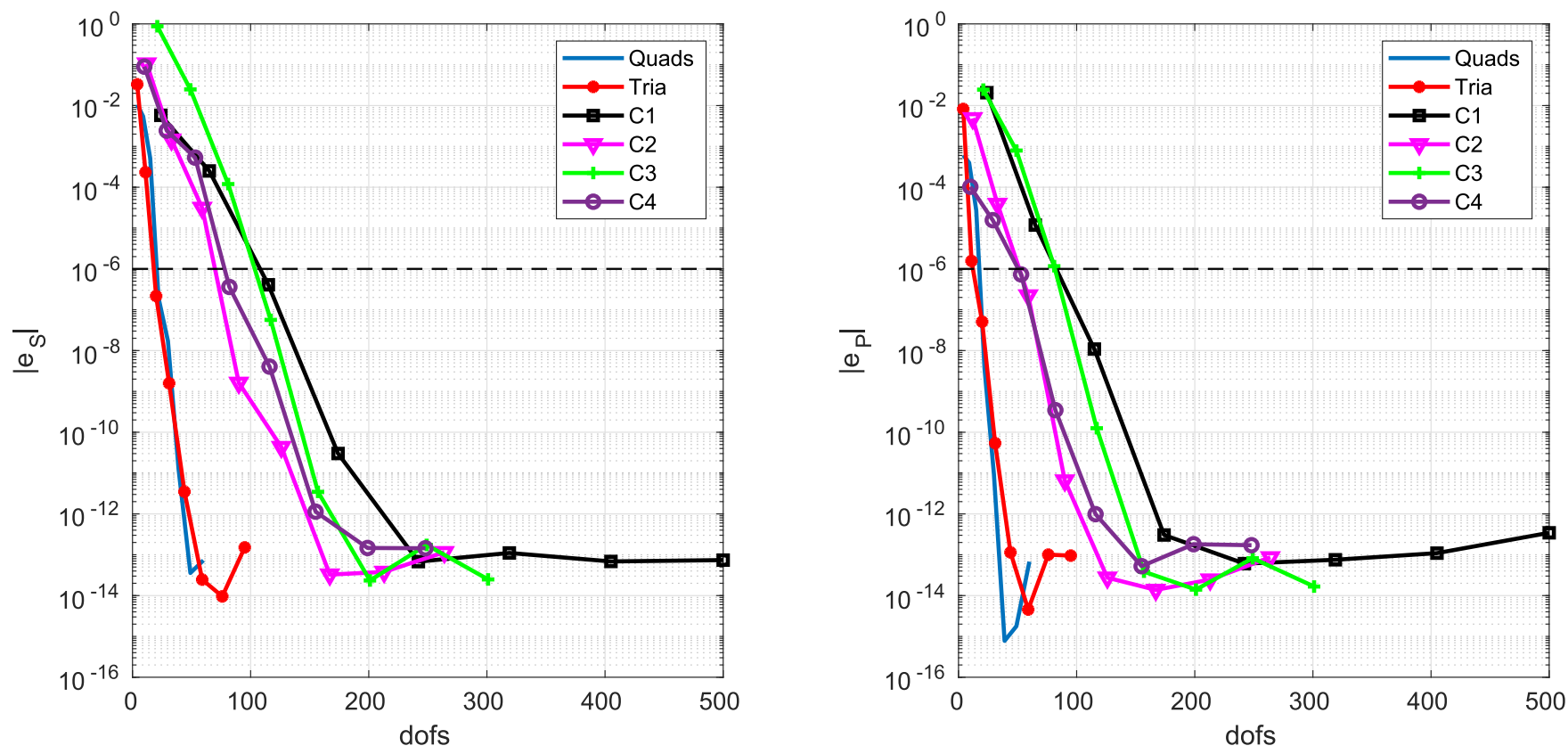

F I G U R E 10 Computed dispersion errors $\left|e_{S}\right|$ and $\left|e_{P}\right|$ as a function of $d o f s$ for $\delta=0.2, \theta=\pi / 4, r=2$ and different mesh configuration. The threshold value $10^{-6}$ is also reported with a black dotted line

the error grows along the direction in which the periodic cell $E_{\text {ref }}$ is split into triangles. This grid orientation effect is in agreement with previous results available in the literature., ${ }^{5,64}$ Composite grids $\mathrm{C} 1, \mathrm{C} 2$, and $\mathrm{C} 3$ seem to perform better than other configurations with respect to all incident angles: this fact can be traced back to the asymmetrical pattern inside the reference cell $E_{\text {ref }}$.

Concerning the dissipation error we study the amplitude of the numerical displacement and take the plane wave $\mathbf{u}(\mathbf{x}, t)=e^{i(\mathbf{k} \cdot \mathbf{x}-\omega t)}$ as exact solution of (3). On the one hand, since $\operatorname{Im}(\omega)=0$, its amplitude is equal to 1 for all times $t$. On the other hand, the numerical approximation will give in general $\operatorname{Im}\left(\omega_{h}\right) \neq 0$. Then, the scheme is nondissipative if 
TA B L E 1 Dispersion errors $\left|e_{P}\right|$ and $\left|e_{S}\right|$ as a function of $k$ with $\delta=0.2, \theta=\pi / 4$

\begin{tabular}{|c|c|c|c|c|c|c|c|}
\hline & & $r=2$ & $r=2$ & $r=5$ & $r=5$ & $r=10$ & $r=10$ \\
\hline Grid & $\boldsymbol{k}$ & $\left|e_{P}\right|$ & $\left|e_{S}\right|$ & $\left|e_{P}\right|$ & $\left|\boldsymbol{e}_{S}\right|$ & $\left|\boldsymbol{e}_{P}\right|$ & $\left|e_{S}\right|$ \\
\hline \multirow[t]{8}{*}{ Quads } & 1 & $6.0577 \mathrm{e}-04$ & $1.0492 \mathrm{e}-02$ & $1.5137 \mathrm{e}-05$ & $1.0492 \mathrm{e}-02$ & $9.4273 e-07$ & $1.0492 \mathrm{e}-02$ \\
\hline & 2 & $3.9826 \mathrm{e}-04$ & $5.4411 \mathrm{e}-03$ & $9.7574 \mathrm{e}-06$ & $1.6753 \mathrm{e}-03$ & $6.0481 \mathrm{e}-07$ & $1.5893 \mathrm{e}-03$ \\
\hline & 3 & $2.7291 \mathrm{e}-05$ & $5.1136 \mathrm{e}-04$ & $6.0824 \mathrm{e}-08$ & $6.3829 \mathrm{e}-05$ & $8.6845 \mathrm{e}-10$ & $6.0938 \mathrm{e}-05$ \\
\hline & 4 & $3.7419 \mathrm{e}-09$ & $1.9706 \mathrm{e}-07$ & $3.8492 \mathrm{e}-13$ & $1.0884 \mathrm{e}-06$ & $6.8826 \mathrm{e}-14$ & $1.0457 \mathrm{e}-06$ \\
\hline & 5 & $9.1927 \mathrm{e}-12$ & $1.7140 \mathrm{e}-08$ & $8.5619 \mathrm{e}-14$ & $1.2832 \mathrm{e}-08$ & $1.9577 \mathrm{e}-13$ & $1.1718 \mathrm{e}-08$ \\
\hline & 6 & $4.4075 \mathrm{e}-15$ & $1.2488 \mathrm{e}-11$ & $1.2882 \mathrm{e}-13$ & $1.2736 \mathrm{e}-10$ & $1.2404 \mathrm{e}-12$ & $1.0704 \mathrm{e}-10$ \\
\hline & 7 & $2.9477 \mathrm{e}-14$ & $3.6223 e-14$ & $3.0194 \mathrm{e}-13$ & $5.1265 \mathrm{e}-13$ & $8.5984 \mathrm{e}-13$ & $4.5778 \mathrm{e}-13$ \\
\hline & 8 & $1.0372 \mathrm{e}-13$ & $7.6169 \mathrm{e}-14$ & $4.6899 \mathrm{e}-13$ & $1.5753 \mathrm{e}-13$ & $8.5753 e-13$ & $9.5340 \mathrm{e}-13$ \\
\hline \multirow[t]{8}{*}{ Tria } & 1 & $8.2437 \mathrm{e}-03$ & $3.3135 \mathrm{e}-02$ & $1.3165 \mathrm{e}-03$ & $3.3135 \mathrm{e}-02$ & $3.2902 \mathrm{e}-04$ & $3.3135 \mathrm{e}-02$ \\
\hline & 2 & $1.5669 \mathrm{e}-06$ & $2.3285 \mathrm{e}-04$ & $5.2817 \mathrm{e}-08$ & $1.8368 \mathrm{e}-04$ & $3.3775 \mathrm{e}-09$ & $1.7914 \mathrm{e}-04$ \\
\hline & 3 & $5.0853 e-08$ & $2.1675 \mathrm{e}-07$ & $1.0534 \mathrm{e}-10$ & $3.1105 e-06$ & $1.8894 \mathrm{e}-12$ & $2.4701 \mathrm{e}-06$ \\
\hline & 4 & $5.4010 \mathrm{e}-11$ & $1.5845 \mathrm{e}-09$ & $8.6515 \mathrm{e}-14$ & $4.7236 \mathrm{e}-09$ & $2.2625 \mathrm{e}-13$ & $4.2769 \mathrm{e}-09$ \\
\hline & 5 & $1.1938 \mathrm{e}-13$ & $3.5012 \mathrm{e}-12$ & $1.5574 \mathrm{e}-13$ & $3.2109 \mathrm{e}-11$ & $7.6318 \mathrm{e}-13$ & $1.5237 \mathrm{e}-11$ \\
\hline & 6 & $1.8481 \mathrm{e}-14$ & $2.6318 \mathrm{e}-14$ & $2.4079 \mathrm{e}-13$ & $3.2615 \mathrm{e}-13$ & $1.2960 \mathrm{e}-12$ & $1.3384 \mathrm{e}-12$ \\
\hline & 7 & $1.0408 \mathrm{e}-13$ & $7.4694 \mathrm{e}-14$ & $5.2719 \mathrm{e}-13$ & $7.0126 \mathrm{e}-13$ & $4.8367 \mathrm{e}-13$ & $5.6891 \mathrm{e}-13$ \\
\hline & 8 & $1.0693 \mathrm{e}-13$ & $1.5421 \mathrm{e}-13$ & $9.3388 \mathrm{e}-13$ & $5.4632 \mathrm{e}-13$ & $1.1880 \mathrm{e}-12$ & $6.9274 \mathrm{e}-13$ \\
\hline
\end{tabular}
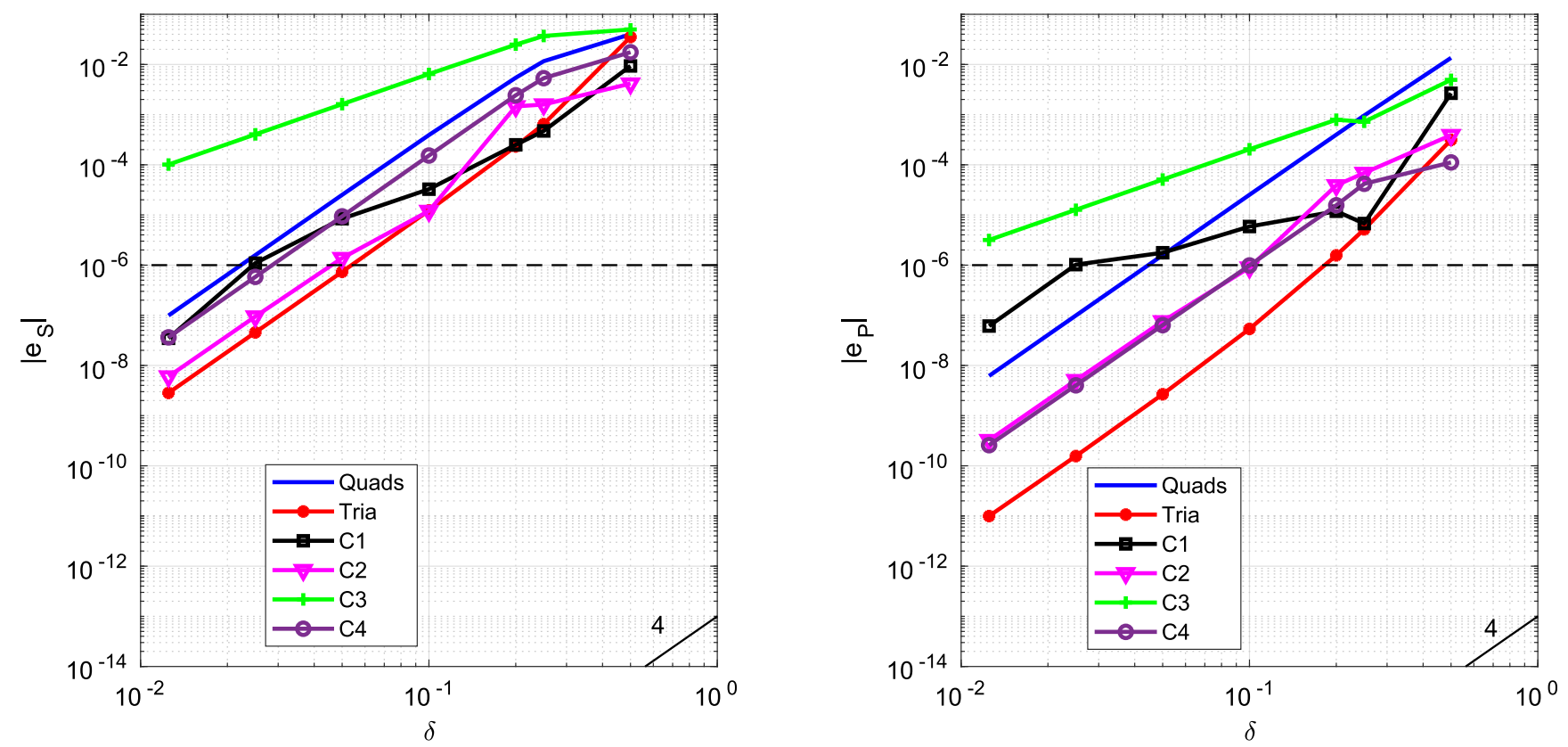

F I G U R E 11 Computed dispersion errors as a function of $\delta$ with $k=2$ for all grid configurations. The threshold value $10^{-6}$ is also reported with a black dotted line 

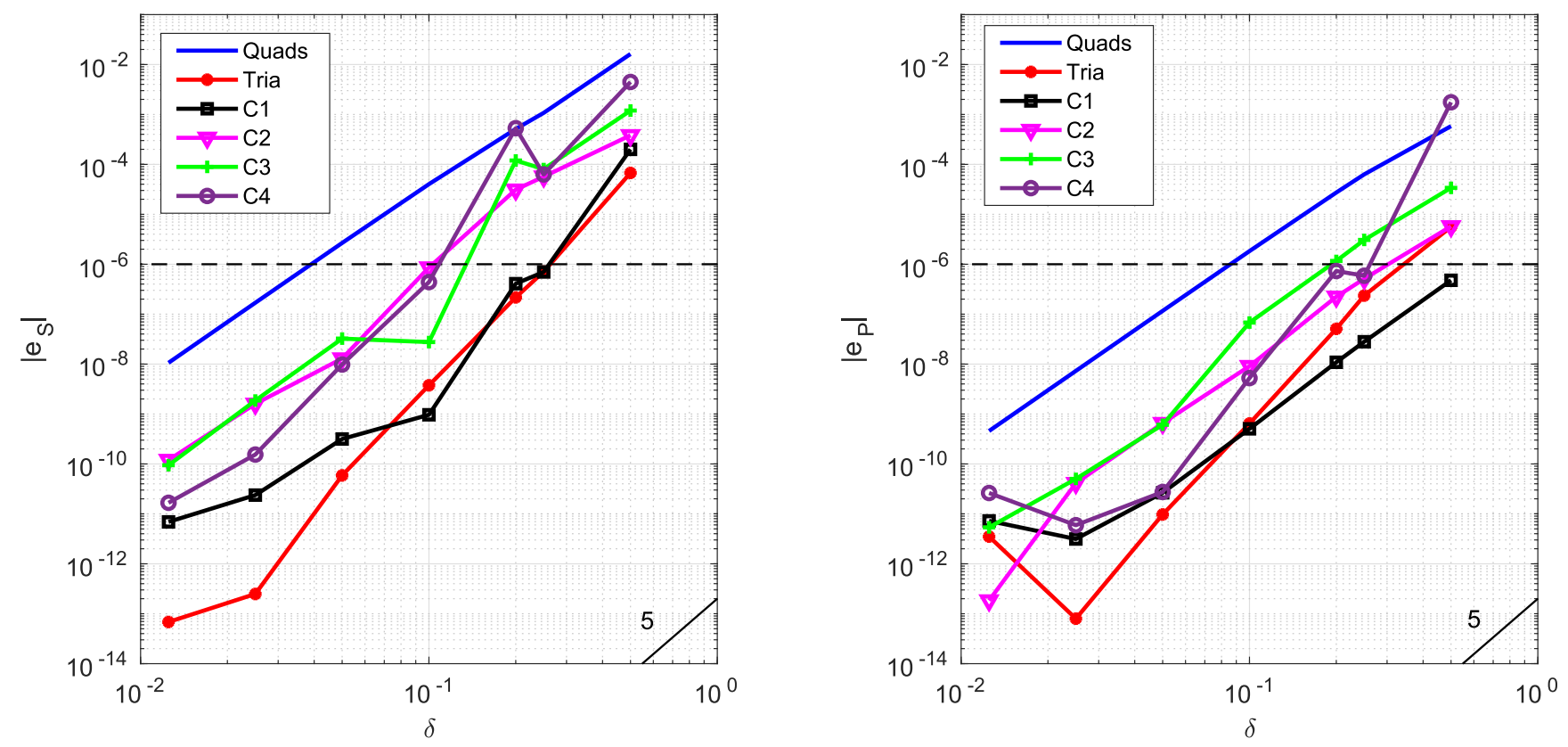

F I G U R E 12 Computed dispersion errors versus $\delta$ with $k=3$ for all grid configurations. The threshold value $10^{-6}$ is also reported with a black dotted line
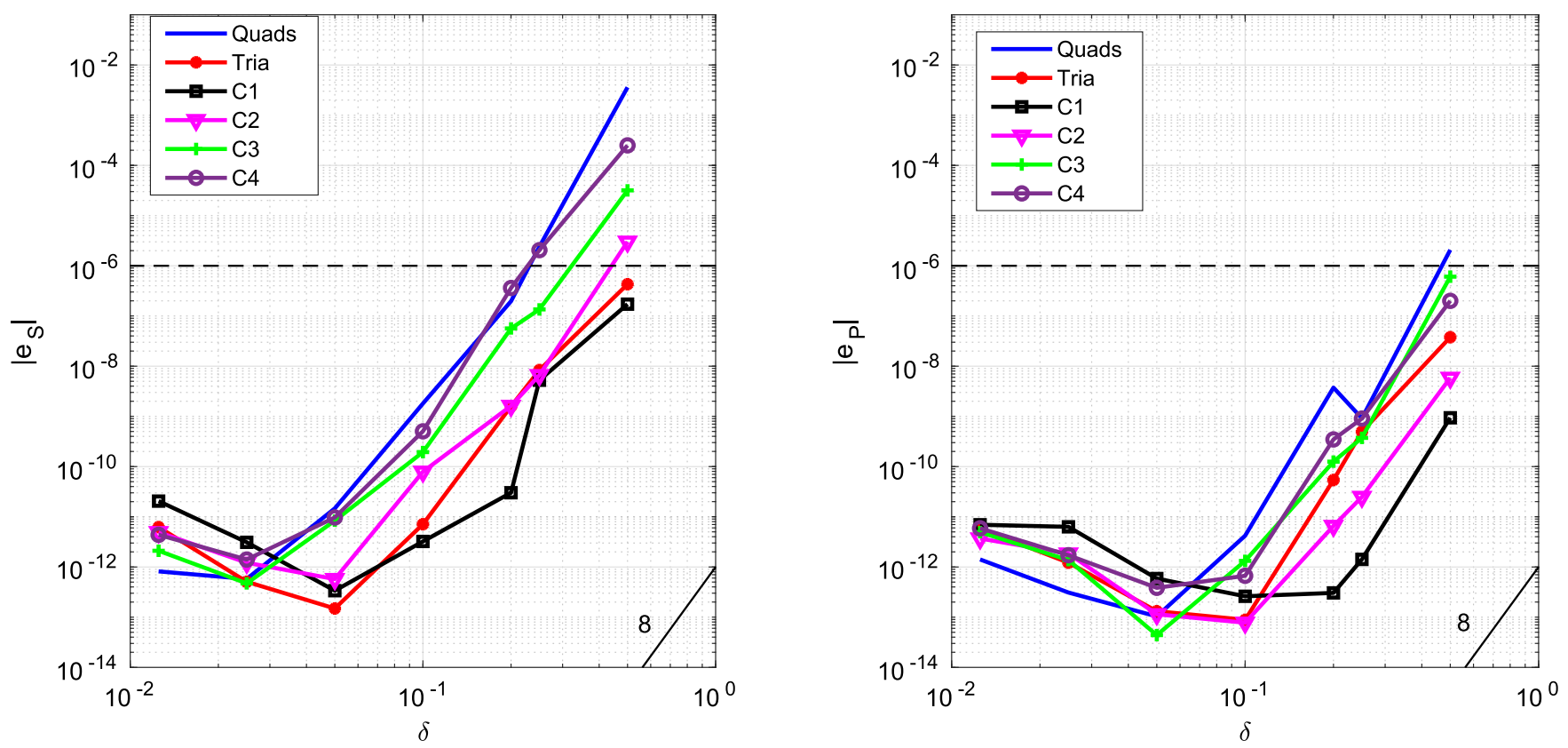

F I G U R E 13 Computed dispersion errors versus $\delta$ with $k=4$ for all grid configurations. The threshold value $10^{-6}$ is also reported with a black dotted line

$\operatorname{Im}\left(\omega_{h}\right)=0$, whereas it is dissipative if $\operatorname{Im}\left(\omega_{h}\right)<0$. In our case, since the mass and the stiffness matrices appearing in (119) are symmetric and positive definite, the computed eigenvalues are all real. This leads to nondissipative schemes.

\subsection{2 | Numerical dispersion and dissipation analysis: Space-time discretization}

We present first some numerical tests for assessing a quantitative estimate of the parameter $q_{C F L}$. In the following, we consider $c_{P}=\sqrt{2}, r=2, \theta \in(0,2 \pi)$ and $\delta=0.2$. Analogous conclusions can be drawn for $r=5$ and $r=10$. 

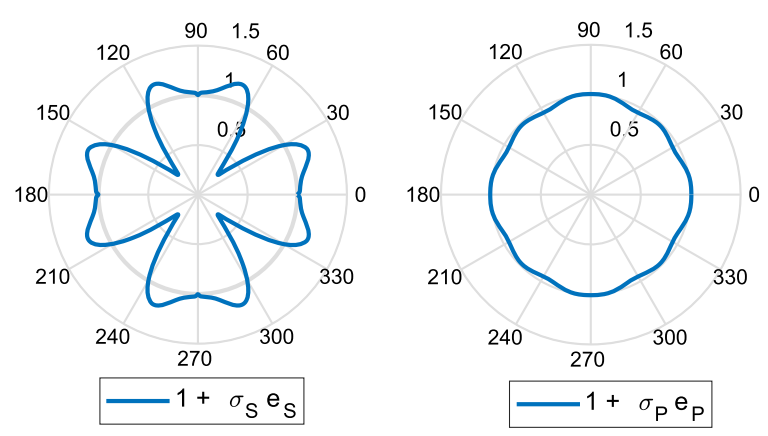

(A) Quadrilateral grid

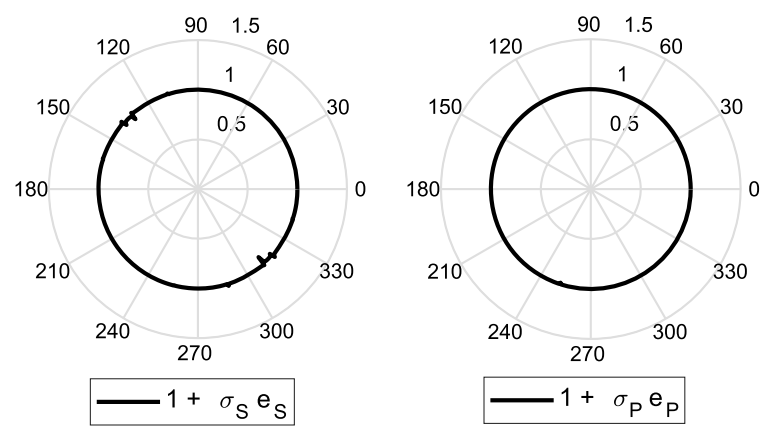

(C) $\mathrm{C} 1$ grid

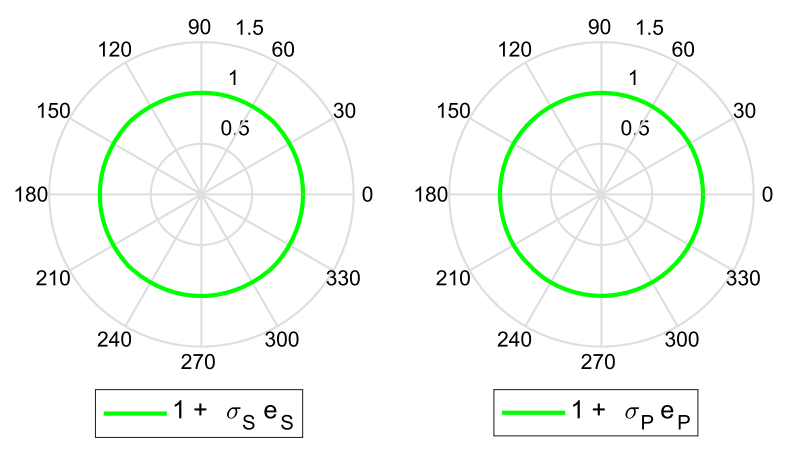

(E) C3 grid

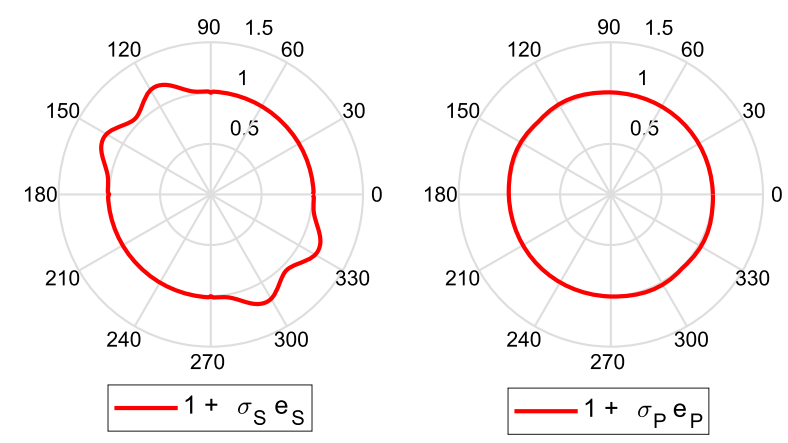

(B) Triangular grid
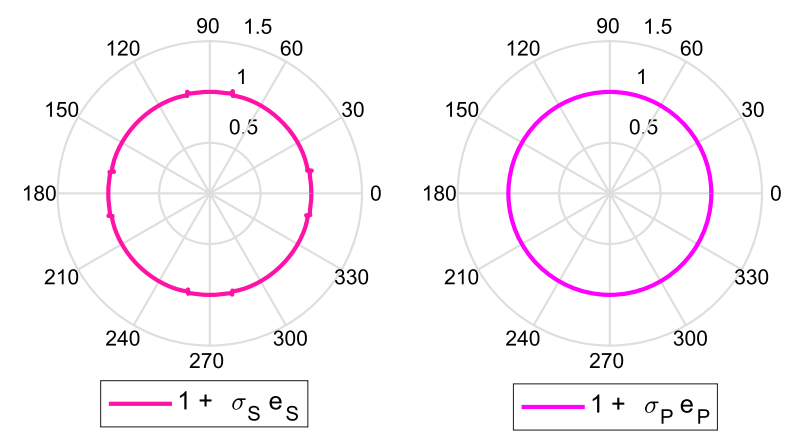

(D) C2 grid

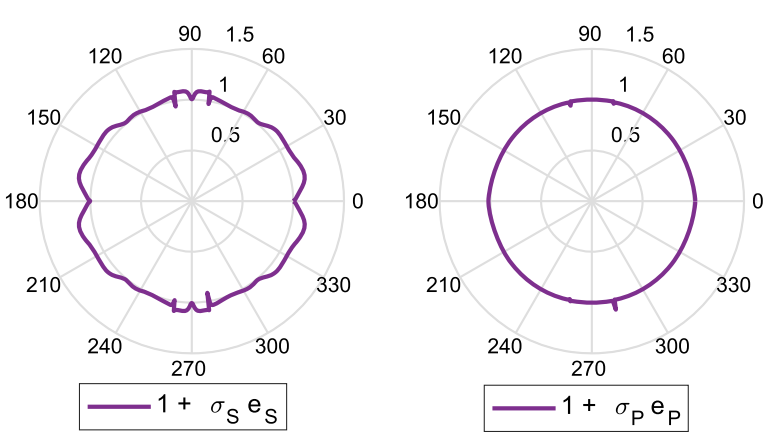

(F) $\mathrm{C} 4$ grid

F I G U R E 14 Anisotropy ratios $c_{S, h} / c_{S}$ (left) and $c_{P, h} / c_{P}$ (right) as a function of the incidence angles $\theta$ for $k=4$ and $\delta=0.5$ for all grid configurations. For visualization purposes dispersion errors are magnified by factors $\sigma_{S}=200$ and $\sigma_{P}=10^{4}$

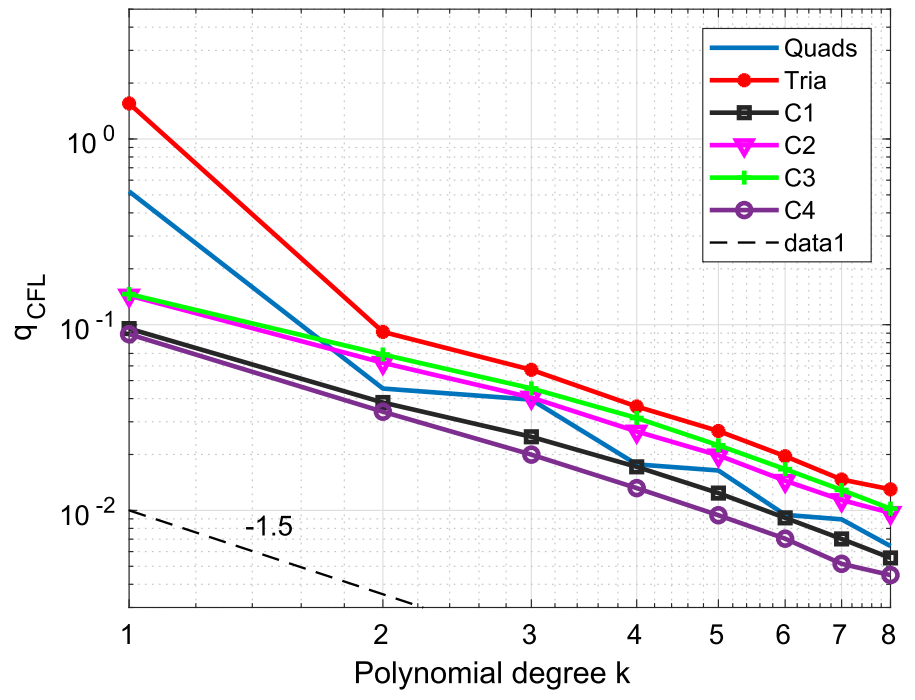

F I G U R E 15 Stability parameter $q_{C F L}$ as a function of the polynomial degree $k$ for all grid configurations 

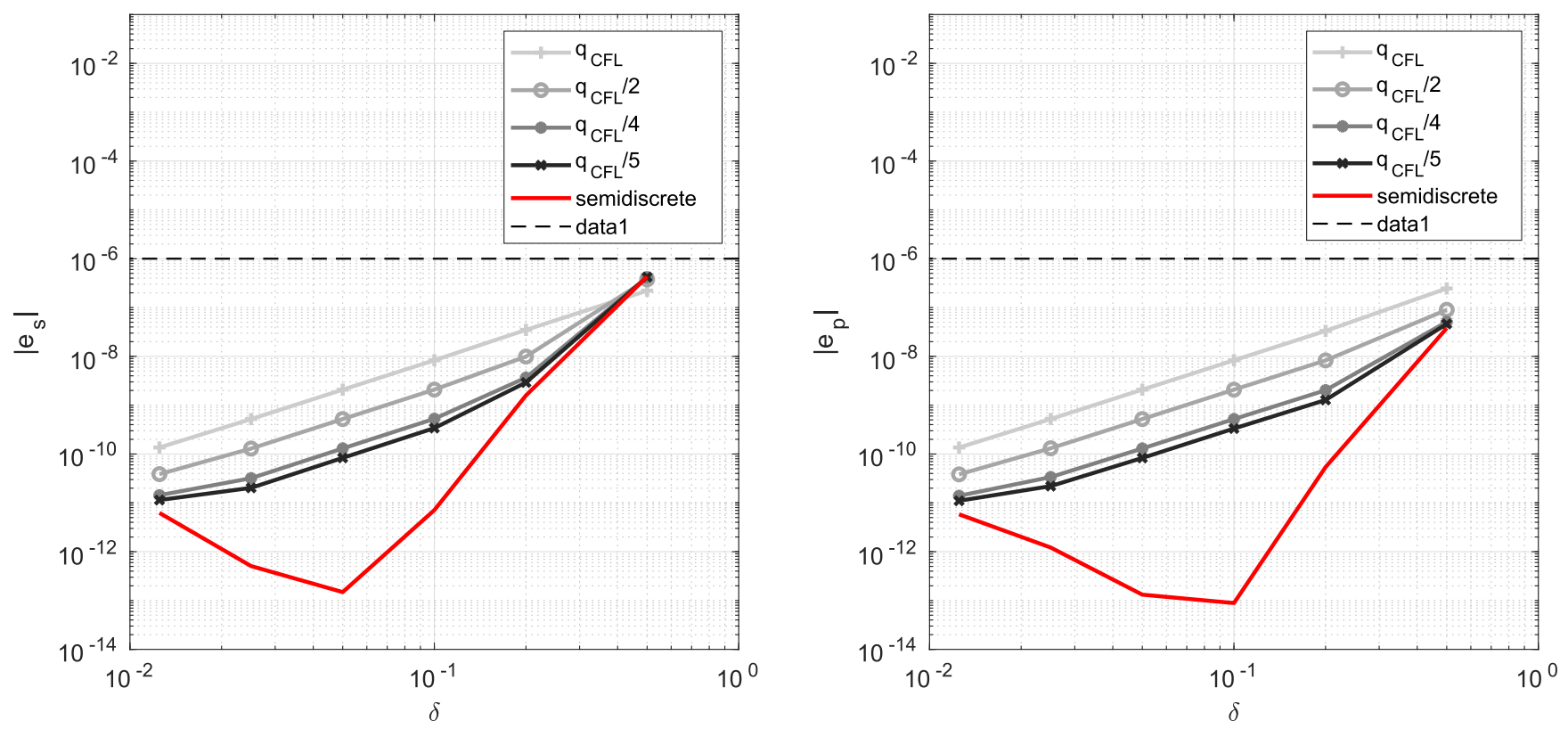

(A) Triangular mesh
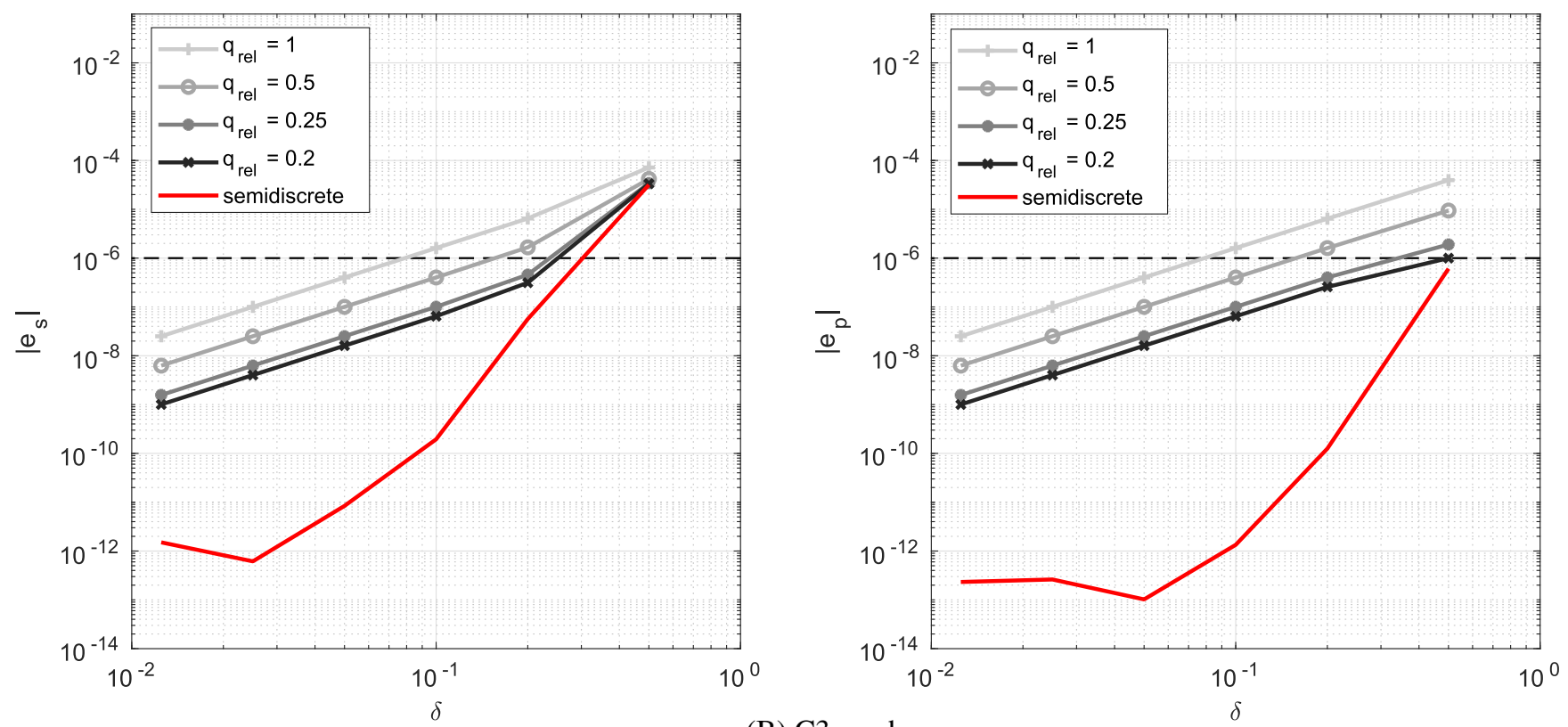

(B) C3 mesh

F I G U R E 16 Computed dispersion errors $\left|e_{S}\right|$ (left) and $\left|e_{P}\right|$ (right) as a function of $\delta$ using triangular (A) and composite C3 (B) grids, with $k=4$. The continuous red lines refer to the semidiscrete approximation, while the others to the fully discrete approximation with $q_{\text {rel }}=0.2,0.25,0.5,1$

Figure 15 shows that the decay rate of $q_{C F L}$ is approximately proportional to $k^{-3 / 2}$ for all the mesh element configurations. This result appears to be slightly better than the one obtained in previous works. ${ }^{5,7,65,68,71}$ In addition, for a given polynomial degree, quadrilateral and $\mathrm{C} 1$ grid elements are subjected to a slightly more restrictive stability condition, that is, lower values of $q_{C F L}$ are obtained.

Next, we present the results of the dispersion and dissipation analysis for the fully discrete approximation by varying the parameters $k, \delta$ and the stability parameter $q$. For the sake of conciseness, we will show only the results related to the triangular and the composite $\mathrm{C} 3$ grids. Similar results have been obtained with the quadrilateral or composite $\mathrm{C} 1, \mathrm{C} 2$, and $\mathrm{C} 4$ meshes. First, we address the behavior of the dispersion error by varying the sampling ratio $\delta$ with fix values of 

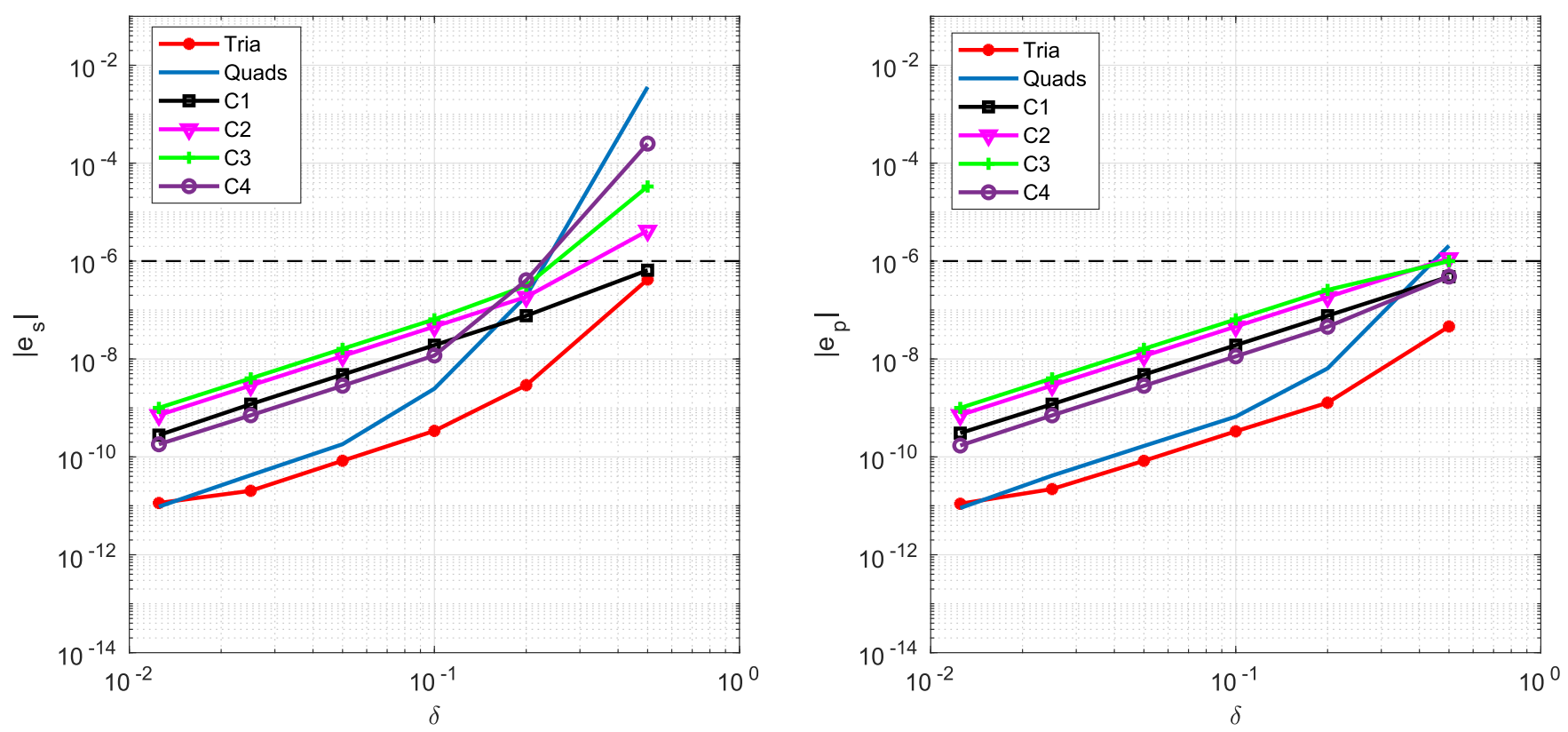

F I G U R E 17 Computed dispersion errors $\left|e_{S}\right|$ (left) and $\left|e_{P}\right|$ (right) as a function of $\delta$, fixing $k=4$ and $q_{r e l}=0.2$

$k=4$ and $\theta=\pi / 4$. The relative stability parameter $q_{r e l}=q / q_{C F L}$ is in the range $(0.2,1)$, and $q_{C F L}$ is computed in agreement with (121). In practice, we first compute $q_{C F L}$ (see (121)) for any specific values $\delta$, cf. also Figure 15; then, we choose $q \in\left(0, q_{C F L}\right)$ and finally we sample the values of the dispersion errors $e_{S}$ and $e_{P}$ as a function of $q$. As $\Delta t$ (and then $q_{\text {rel }}$ ) goes to 0 , the fully discrete curves tend to the semidiscrete ones, see Figure 16. In Figure 17, we compare the results obtained with all mesh element configurations for $q_{r e l}=0.2$. On all the considered grids, the VEM reaches the same level of accuracy, and, in particular $\delta \leq 0.2$, that is, five points per wavelength, are sufficient to obtain dispersion errors lower than $10^{-6}$.

Next, by letting varying the polynomial degree $k$ for $\delta=0.2$ and $\theta=\pi / 4$ we analyze the dispersion error. In Figure 18, we observe the exponential convergence that was noted in the semidiscrete case, cf. Figure $9, q_{\text {rel }}$ goes to zero. Indeed, for sufficiently small values of $q$, the following asymptotic relation holds. ${ }^{71}$

$$
\omega_{h} \approx \sqrt{\Lambda}+\mathcal{O}\left(\Delta t^{2}\right)
$$

Thus $\omega_{h}$ decays as in the semidiscrete case until the term $\Delta t^{2}$ becomes dominant. In Figure 19, we compare the behavior of the fully discrete scheme obtained on all the considered grids for $q_{r e l}=0.2$. We notice that for all the grid configurations the same level of accuracy is achieved with a polynomial degree $k \geq 4$, with a better performance of triangular and quadrilateral grids.

Regarding the dissipation error of the space-time discretized scheme, the considerations made for the space discretized case remain valid.

\section{6 | CONCLUSIONS}

In this work, we extended the conforming virtual element method for the numerical simulation of two-dimensional time-dependent elastodynamics problems. The formulation of the VEM is investigated both theoretically and numerically. From the theoretical side, we proved the stability and the convergence of the semidiscrete approximation in the energy norm and obtain optimal rate of convergence. We also derive $L^{2}$ error estimates with optimal convergence rate for both the $h$ - and $p$-refinement. From the numerical side, we assessed the accuracy of the conforming VEM on a set of different computational meshes, including nonconvex cells. Optimal convergence rates in the energy 

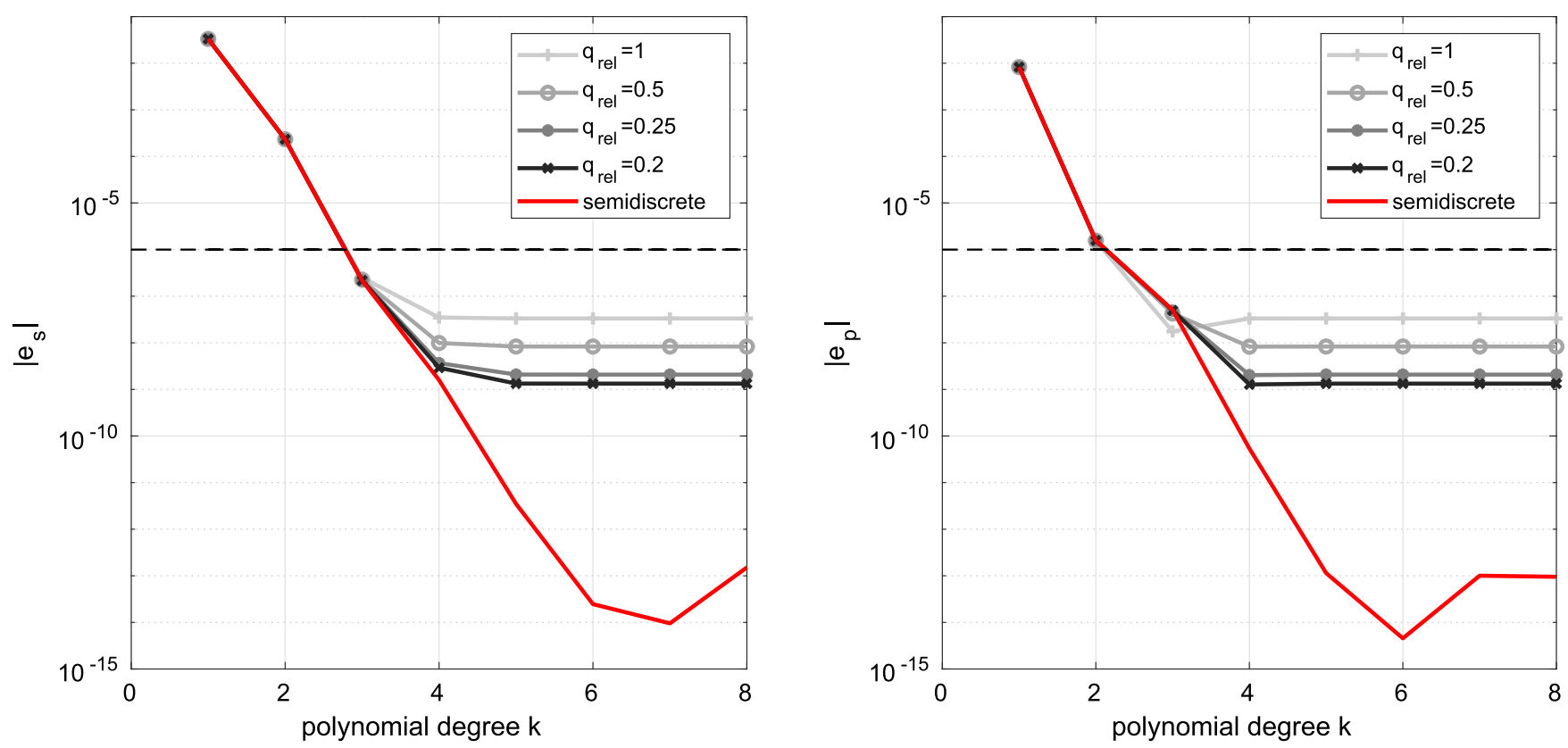

(A) Triangular mesh
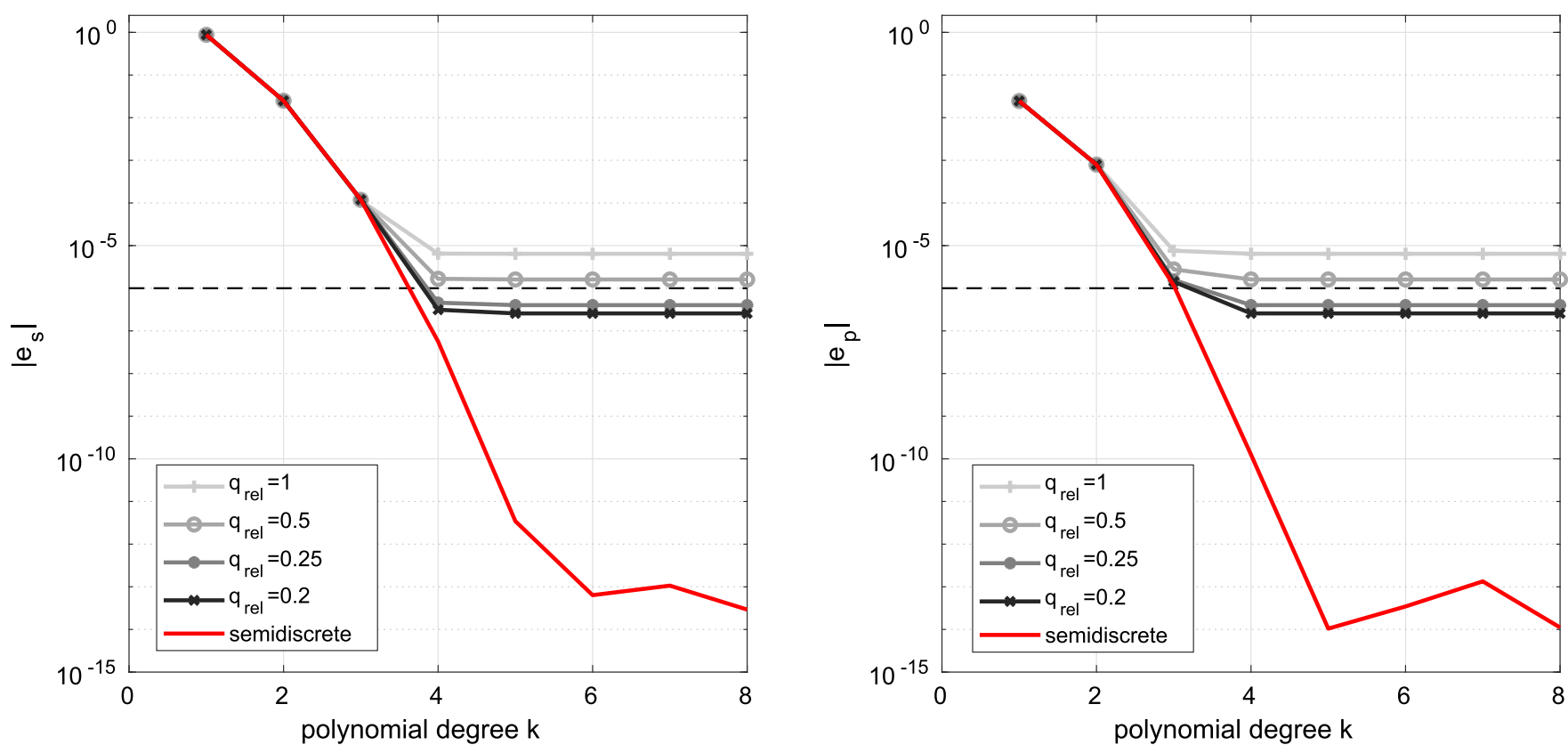

(B) $\mathrm{C} 3$ mesh

F I G U R E 18 Computed dispersion errors $\left|e_{S}\right|$ (left) and $\left|e_{P}\right|$ (right) as a function of $k$ using triangular (A) and composite C3 (B) grids, with a fixed sampling ratio $\delta=0.2$. The red lines refer to the semidiscrete approximation, while the others to the fully discrete approximation with $q_{\text {rel }}=0.2,0.25,0.5,1$

norm and $L^{2}$ norm in the $h$-refinement are numerically validated, and exponential convergence is experimentally observed in both norms in the $p$-refinement setting. Moreover, a thorough comparison with standard VEM schemes on simplicial/quadrilateral grids is presented in term of a dispersion-dissipation and stability analysis, showing that polygonal meshes behave as classical simplicial/quadrilateral grids in terms of dispersion-dissipation and stability properties. 

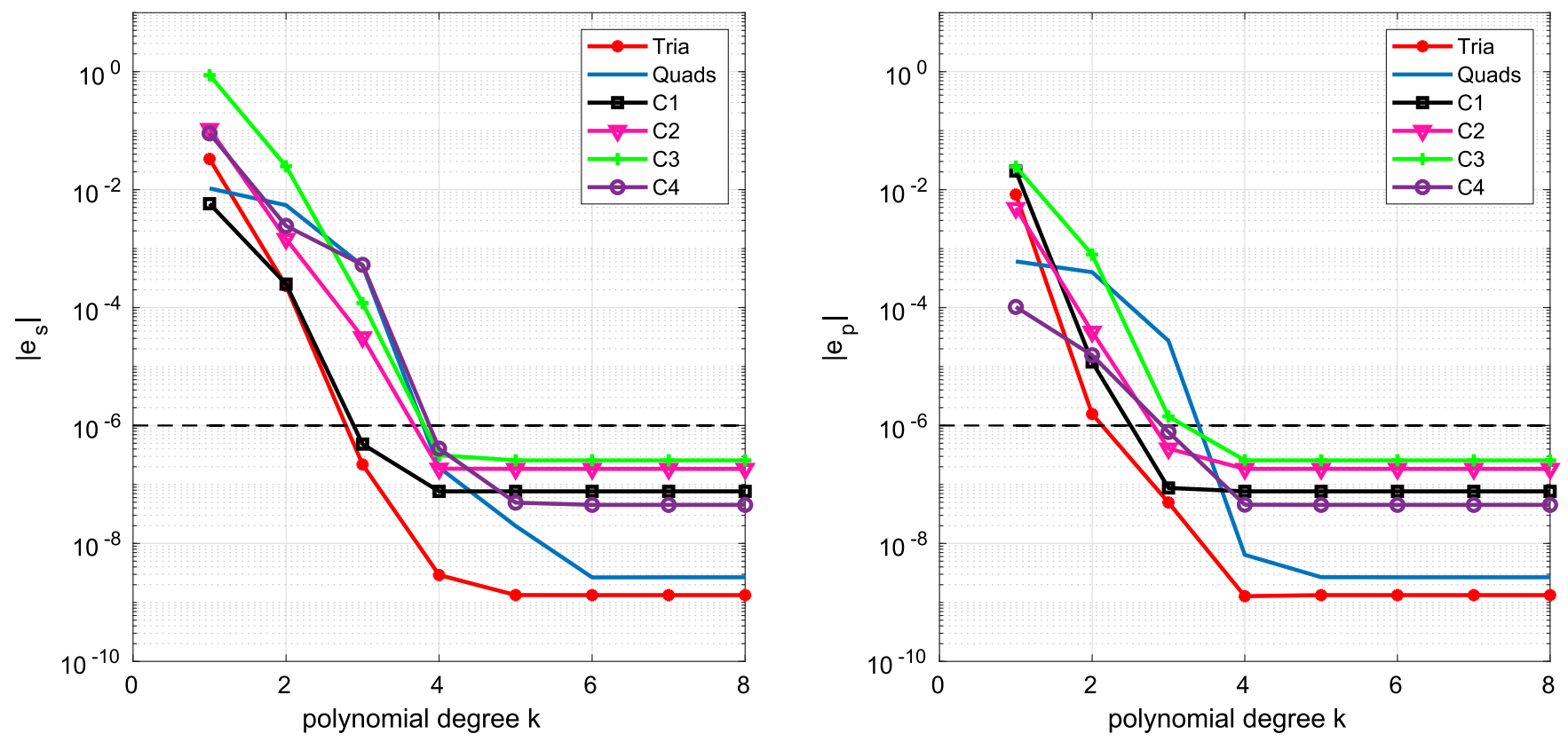

F I G U R E 19 Computed dispersion errors $\left|e_{S}\right|$ (left) and $\left|e_{P}\right|$ (right) as a function of $k$, for $\delta=0.2$ and $q_{\text {rel }}=0.2$

\section{ACKNOWLEDGMENTS}

The authors are grateful to the anonymous referees for their valuable and constructive comments, which have contributed to the improvement of the article. The authors also thank Dr. Lorenzo Mascotto (University of Vienna) for a useful discussion on the content of Remark 5. Paola F. Antonietti and Marco Verani acknowledge the financial support of PRIN research grant number 201744KLJL "Virtual Element Methods: Analysis and Applications" funded by MIUR. Paola F. Antonietti, Ilario Mazzieri, and Marco Verani also acknowledge the financial support of INdAM-GNCS. The work of Gianmarco Manzini was supported by the Laboratory Directed Research and Development (LDRD) Program of Los Alamos National Laboratory under project number 20180428ER. The work of Hashem M. Mourad was supported by the LDRD program of Los Alamos National Laboratory under project number 20170033DR. Los Alamos National Laboratory is operated by Triad National Security, LLC, for the National Nuclear Security Administration of U.S. Department of Energy (Contract No. 89233218CNA000001).

\section{ORCID}

Gianmarco Manzini (ํ) https://orcid.org/0000-0003-3626-3112

\section{REFERENCES}

1. Komatitsch D, Tromp J. Introduction to the spectral element method for three-dimensional seismic wave propagation. Geophys $J$ Int. 1999;139:806-822.

2. Faccioli E, Maggio F, Quarteroni A, Tagliani A. Spectral-domain decomposition methods for the solution of acoustic and elastic wave equations. Geophysics. 1996;61:1160-1174.

3. Rivière B, Wheeler MF. Discontinuous finite element methods for acoustic and elastic wave problems. Contemp Math. 2003;329:271-282.

4. Antonietti PF, Ayuso de Dios B, Mazzieri I, Quarteroni A. Stability analysis of discontinuous Galerkin approximations to the elastodynamics problem. J Sci Comput. 2016;68:143-170.

5. Antonietti PF, Mazzieri I, Quarteroni A, Rapetti F. Non-conforming high order approximations of the Elasto dynamics equation. Comput Methods Appl Mech Eng. 2012;209:212-238.

6. Dumbser M, Käser M. An arbitrary high-order discontinuous Galerkin method for elastic waves on unstructured meshes - II. the three-dimensional isotropic case. Geophys J Int. 2006;167:319-336.

7. Antonietti PF, Mazzieri I. High-order discontinuous Galerkin methods for the elastodynamics equation on polygonal and polyhedral meshes. Comput Methods Appl Mech Eng. 2018;342:414-437.

8. Antonietti PF, Bonaldi F, Mazzieri I. A high-order discontinuous Galerkin approach to the elasto-acoustic problem. Comput Methods Appl Mech Eng. 2020;358:112634. 
9. Park K, Chi H, Paulino GH. On nonconvex meshes for elastodynamics using virtual element methods with explicit time integration. Comput Methods Appl Mech Eng. 2019;356:669-684.

10. Park K, Chi H, Paulino GH. Numerical recipes for elastodynamic virtual element methods with explicit time integration. Int J Numer Methods Eng. 2020;121:1-31.

11. Beirão da Veiga L, Lipnikov K, Manzini G. The Mimetic Finite Difference Method. volume 11 of. Modeling, Simulations and Applications. I st ed. New York, NY: Springer; 2014.

12. Beirão da Veiga L, Brezzi F, Cangiani A, Manzini G, Marini LD, Russo A. Basic principles of virtual element methods. Math Models Methods Appl Sci. 2013;23:119-214.

13. Ayuso de Dios B, Lipnikov K, Manzini G. The non-conforming virtual element method. ESAIM Math Modell Numer Anal. 2016;50:879-904.

14. Cangiani A, Manzini G, Sutton O. Conforming and nonconforming virtual element methods for elliptic problems. IMA J Numer Anal. 2017;37:1317-1354.

15. Cangiani A, Gyrya V, Manzini G. The non-conforming virtual element method for the Stokes equations. SIAM J Numer Anal. 2016;54:3411-3435.

16. Antonietti PF, Manzini G, Verani M. The fully nonconforming virtual element method for biharmonic problems. Math Models Methods Appl Sci. 2018;28:387-407.

17. Mascotto L, Perugia I, Pichler A. A nonconforming Trefftz virtual element method for the Helmholtz problem. Math Models Methods Appl Sci. 2019;22:1619-1656.

18. Antonietti PF, Manzini G, Verani M. The conforming virtual element method for polyharmonic problems. Comput Math Appl. 2019;79:2021-2034.

19. Beirão da Veiga L, Manzini G, Mascotto L. A posteriori error estimation and adaptivity in hp virtual elements. Numer Math. 2019;143:139-175.

20. Manzini G, Russo A, Sukumar N. New perspectives on polygonal and polyhedral finite element methods. Math Models Methods Appl Sci. 2014;24:1621-1663.

21. Brezzi F, Marini LD. Virtual element methods for plate bending problems. Comput Methods Appl Mech Eng. 2013;253:455-462.

22. Beirão da Veiga L, Brezzi F, Marini LD. Virtual elements for linear elasticity problems. SIAM J Numer Anal. 2013;51:794-812.

23. Artioli E, de Miranda S, Lovadina C, Patruno L. A stress/displacement virtual element method for plane elasticity problems. Comput Methods Appl Mech Eng. 2017;325:155-174.

24. Artioli E, de Miranda S, Lovadina C, Patruno L. A family of virtual element methods for plane elasticity problems based on the Hellinger-Reissner principle. Comput Methods Appl Mech Eng. 2018;340:978-999.

25. Cáceres E, Gatica GN, Sequeira FA. A mixed virtual element method for a pseudostress-based formulation of linear elasticity. Appl Numer Math. 2019;135:423-442.

26. Zhang B, Zhao J, Yang Y, Chen S. The nonconforming virtual element method for elasticity problems. J Comput Phys. 2018;378: 349-410.

27. Gain AL, Talischi C, Paulino GH. On the virtual element method for three-dimensional linear elasticity problems on arbitrary polyhedral meshes. Comput Methods Appl Mech Eng. 2014;282:132-160.

28. De Bellis ML, Wriggers P, Hudobivnik B. Serendipity virtual element formulation for nonlinear elasticity. Comput Struct. 2019;223:106094.

29. Wriggers P, Rust WT, Reddy BD. A virtual element method for contact. Comput Mech. 2016;58:1039-1050.

30. Wriggers P, Rust WT. A virtual element method for frictional contact including large deformations. Eng Comput. 2019;36:2133-2161.

31. Beirão da Veiga L, Lovadina C, Mora D. A virtual element method for elastic and inelastic problems on polytope meshes. Comput Methods Appl Mech Eng. 2015;295:327-346.

32. Wriggers P, Reddy BD, Rust W, Hudobivnik B. Efficient virtual element formulations for compressible and incompressible finite deformations. Comput Mech. 2017;60:253-268.

33. Chi H, Beirão da Veiga L, Paulino GH. Some basic formulations of the virtual element method (VEM) for finite deformations. Comput Methods Appl Mech Eng. 2017;318:148-192.

34. Wriggers P, Hudobivnik B. A low order virtual element formulation for finite elasto-plastic deformations. Comput Methods Appl Mech Eng. 2017;327:459-477.

35. Hudobivnik B, Aldakheel F, Wriggers P. A low order 3D virtual element formulation for finite Elasto plastic deformations. Comput Mech. 2018;63:253-269.

36. Dhanush V, Natarajan S. Implementation of the virtual element method for coupled thermo-elasticity in Abaqus. Numer Algorithms. 2018;80:1037-1058.

37. Mora D, Rivera G. A priori and a posteriori error estimates for a virtual element spectral analysis for the elasticity equations. IMA J Numer Anal. 2018;40:322-357.

38. Reddy BD, van Huyssteen D. A virtual element method for transversely isotropic elasticity. Comput Mech. 2019;64:971-988.

39. Vacca G. Virtual element methods for hyperbolic problems on polygonal meshes. Comput Math Appl. 2016;74:882-898.

40. Adams RA, Fournier JJF. Sobolev Spaces. Pure and Applied Mathematics. 2nd ed. Amsterdam: Academic Press; 2003.

41. Raviart PA, Thomas JM. Introduction à l'analyse numérique des équations aux dérivées partielles. Collection Mathématiques Appliquées pour la Maitrise [Collection of Applied Mathematics for the Master's Degree]. Paris: Masson; 1983.

42. Quarteroni A, Sacco R, Saleri F. Numerical Mathematics. Vol. 37 of Texts in Applied Mathematics. New York, NY: Springer; 2007.

43. Ahmad B, Alsaedi A, Brezzi F, Marini LD, Russo A. Equivalent projectors for virtual element methods. Comput Math Appl. 2013;66:376-391. 
44. Beirão da Veiga L, Lovadina C, Russo A. Stability analysis for the virtual element method. Math Models Methods Appl Sci. 2017;27:2557-2594.

45. Brenner SC, Sung LY. Virtual element methods on meshes with small edges or faces. Math Models Methods Appl Sci. 2018;28:1291-1336.

46. Cao S, Chen L. Anisotropic error estimates of the linear virtual element method on polygonal meshes. SIAM J Numer Anal. 2018;56:2913-2939.

47. Mascotto L. Ill-conditioning in the virtual element method: stabilizations and bases. Numer Methods Partial Differ Equat. 2018;34:1258-1281.

48. Dassi F, Mascotto L. Exploring high-order three dimensional virtual elements: bases and stabilizations. Comput Math Appl. 2018;75:3379-3401.

49. Beirão da Veiga L, Chernov A, Mascotto L, Russo A. Basic principles of hp virtual elements on quasiuniform meshes. Math Models Methods Appl Sci. 2016;26:1567-1598.

50. Beirão da Veiga L, Brezzi F, Marini LD, Russo A. The Hitchhiker's guide to the virtual element method. Math Models Methods Appl Sci. 2014;24:1541-1573.

51. Certik O, Gardini F, Manzini G, Mascotto L, Vacca G. The p- and hp-versions of the virtual element method for elliptic eigenvalue problems. Comput Math Appl. 2019;79:2035-2056.

52. Beirão da Veiga L, Chernov A, Mascotto L, Russo A. Exponential convergence of the $h p$ virtual element method in presence of corner singularities. Numer Math. 2018;138:581-613.

53. Brezis H. Operateurs maximaux monotones et semi-groupes de contractions dans les espaces de Hilbert. Mathematical Studies. Amsterdam: North-Holland Publishing Company; 1973.

54. Baker GA, Dougalis VA, Karakashian OA. On multistep-Galerkin discretizations of semilinear hyperbolic and parabolic equations. Nonlinear Anal Theory Methods Appl. 1980;4:579-597.

55. Adak D, Natarajan S. Virtual element method for semilinear sine-Gordon equation over polygonal mesh using product approximation technique. Math Comput Simul. 2020;172:224-243.

56. Vacca G, Beirão da Veiga L. Virtual element methods for parabolic problems on polygonal meshes. Numer Methods Partial Differ Equat Int J. 2015;31:2110-2134.

57. Antonietti PF, Manzini G, Mazzieri I, Mourad H, Verani M. The arbitrary-order virtual element method for linear elastodynamics models. Convergence, stability and dispersion-dissipation analysis. An extended version; 2020. arXiv article N. arXiv:1912.07122v5.

58. Beirão da Veiga L, Lipnikov K, Manzini G. Arbitrary order nodal mimetic discretizations of elliptic problems on polygonal meshes. SIAM J Numer Anal. 2011;49:1737-1760.

59. Berrone S, Borio A. Orthogonal polynomials in badly shaped polygonal elements for the virtual element method. Finite Elements Anal Des. 2017;129:14-31.

60. Cohen GC. Higher-Order Numerical Methods for Transient Wave Equations. Scientific Computation. 1st ed. Berlin, Heidelberg: Springer-Verlag; 2002.

61. Ainsworth M. Discrete dispersion relation for $h p$-version finite element approximation at high wave number. SIAM $J$ Numer Anal. 2004;42:553-575.

62. Ainsworth M. Dispersive and dissipative behaviour of high order discontinuous Galerkin finite element methods. J Comput Phys. 2004;198:106-130.

63. Eringen AC, Suhubi ES. Elastodynamics. Vol. 2: Linear Theory. New York, London, UK: Academic Press; 1975.

64. Mazzieri I, Rapetti F. Dispersion analysis of triangle-based spectral element methods for elastic wave propagation. Numer Algorithms. 2012;60:631-650.

65. Ferroni A, Antonietti PF, Mazzieri I, Quarteroni A. Dispersion-dissipation analysis of 3-D continuous and discontinuous spectral element methods for the elastodynamics equation. Geophys J Int. 2017;211:1554-1574.

66. Alford RM, Kelly KR, Boore DM. Accuracy of finite-difference modeling of the acoustic wave equation. Geophysics. 1974;39:834-842.

67. De Basabe JD, Sen MK. Grid dispersion and stability criteria of some common finite-element methods for acoustic and elastic wave equations. Geophysics. 2007;72:T81-T95.

68. De Basabe JD, Sen MK. Stability of the high-order finite elements for acoustic or elastic wave propagation with high-order time stepping. Geophys J Int. 2010;181:577-590.

69. Antonietti PF, Houston P. A class of domain decomposition preconditioners for $h p$-discontinuous Galerkin finite element methods. $J$ Sci Comput. 2011;46:124-149.

70. De Basabe JD, Sen MK, Wheeler MF. The interior penalty discontinuous Galerkin method for elastic wave propagation: grid dispersion. Geophys J Int. 2008;175:83-93.

71. Antonietti PF, Marcati C, Mazzieri I, Quarteroni A. High order discontinuous Galerkin methods on simplicial elements for the elastodynamics equation. Numer Algorithms. 2016;71:181-206.

How to cite this article: Antonietti PF, Manzini G, Mazzieri I, Mourad HM, Verani M. The arbitrary-order virtual element method for linear elastodynamics models: convergence, stability and dispersion-dissipation analysis. Int J Numer Methods Eng. 2020;1-38. https://doi.org/10.1002/nme.6569 